Pacific

Journal of

Mathematics

SINGULARITY REMOVABILITY AT BRANCH POINTS FOR WILLMORE SURFACES

YANN BERNARD AND TRISTAN RIVIÈRE 


\title{
SINGULARITY REMOVABILITY AT BRANCH POINTS FOR WILLMORE SURFACES
}

\author{
YANN BERNARD AND TRISTAN RIVIÈRE
}

\begin{abstract}
We consider a branched Willmore surface immersed in $\mathbb{R}^{m \geq 3}$ with squareintegrable second fundamental form. We develop around each branch point local asymptotic expansions for the Willmore immersion, its first, and its second derivatives. Our expansions are given in terms of new integer-valued residues which are computed as circulation integrals around the branch point. We deduce explicit "point removability" conditions guaranteeing that the immersion is smooth through the branch point. These conditions are new, even in codimension one.
\end{abstract}

\section{Introduction}

1A. Preliminaries. Let $\vec{\Phi}$ be an immersion from a closed abstract two-dimensional manifold $\Sigma$ into $\mathbb{R}^{m \geq 3}$. We denote by $g:=\vec{\Phi}^{*} g_{\mathbb{R}^{m}}$ the pullback by $\vec{\Phi}$ of the flat canonical metric $g_{\mathbb{R}^{m}}$ of $\mathbb{R}^{m}$, also called the first fundamental form of $\vec{\Phi}$, and we let $d \mathrm{vol}_{\mathrm{g}}$ be its associated volume form. The Gauss map of the immersion $\vec{\Phi}$ is the map taking values in the Grassmannian of oriented $(m-2)$-planes in $\mathbb{R}^{m}$ given by

$$
\vec{n}:=\star \frac{\partial_{x_{1}} \vec{\Phi} \wedge \partial_{x_{2}} \vec{\Phi}}{\left|\partial_{x_{1}} \vec{\Phi} \wedge \partial_{x_{2}} \vec{\Phi}\right|},
$$

where $\star$ is the usual Hodge star operator in the Euclidean metric, and $\left\{x_{1}, x_{2}\right\}$ are local coordinates on the surface $\Sigma$.

Denoting by $\pi_{\vec{n}}$ the orthonormal projection of vectors in $\mathbb{R}^{m}$ onto the $(m-2)$ plane given by $\vec{n}$, the second fundamental form may be expressed as

$$
\overrightarrow{\mathbb{1}}_{p}(X, Y):=\pi_{\vec{n}} d^{2} \vec{\Phi}(X, Y) \text { for all } X, Y \in T_{p} \Sigma .
$$

(In order to define $d^{2} \vec{\Phi}(X, Y)$ one has to extend locally around $T_{p} \Sigma$ the vector

Bernard is supported by the DFG Collaborative Research Center SFB/Transregio 71 (Project B3). Parts of this work were completed during his visits to the welcoming facilities of the Forschungsinstitut für Mathematik at the ETH in Zurich.

MSC2010: primary 30C70, 35J35, 35J50, 35J48, 35R01; secondary 49Q10, 53A30, 32S25, 58E15, $58 \mathrm{E} 30$. 
fields $X$ and $Y$. It is not difficult to check that $\pi_{\vec{n}} d^{2} \vec{\Phi}(X, Y)$ is independent of this extension.)

The mean curvature vector of the immersion at the point $p \in \Sigma$ is

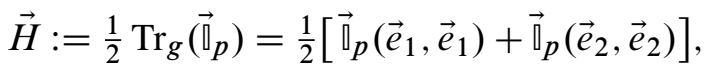

where $\left\{\vec{e}_{1}, \vec{e}_{2}\right\}$ is an orthonormal basis of $T_{p} \Sigma$ for the metric $g$.

In the present paper, we study the functional

$$
W(\vec{\Phi}):=\int_{\Sigma}|\vec{H}|^{2} d \operatorname{vol}_{g}
$$

called Willmore energy. It has been extensively studied in the literature, due to its relevance to various areas of science. We refer the reader to [Rivière 2010] and the references therein for more extensive information on the properties and applications of the Willmore energy.

The Gauss-Bonnet theorem and Gauss equation imply that

$$
W(\vec{\Phi})=\frac{1}{4} \int_{\Sigma}|\vec{q}|_{g}^{2} d \operatorname{vol}_{g}+\pi \chi(\Sigma)=\frac{1}{4} \int_{\Sigma}|d \vec{n}|_{g}^{2} d \operatorname{vol}_{g}+\pi \chi(\Sigma),
$$

where $\chi(\Sigma)$ is the Euler characteristic of $\Sigma$, which is a topological invariant for a closed surface. From the variational point of view, the critical points of the Willmore functional, called Willmore surfaces, are thus also critical points of the Dirichlet energy of the Gauss map with respect to the induced metric $g$.

Minimal surfaces ${ }^{1}$ are examples of Willmore surfaces. Not only is the Willmore energy invariant under reparametrization of the domain, but, more remarkably, it is invariant under Möbius transformations of $\mathbb{R}^{m} \cup\{\infty\}$; namely,

$$
W(\Xi \circ \vec{\Phi})=W(\vec{\Phi}) \text { for any conformal diffeomorphism } \Xi \text { of } \mathbb{R}^{m} \cup\{\infty\} .
$$

Hence, the image of a Willmore immersion by a conformal transformation is again a Willmore immersion. It is thus no surprise that the class of Willmore immersions is considerably larger than that of minimal immersions (whose minimality is not preserved through conformal diffeomorphism).

An important task in the analysis of Willmore surfaces is to understand the closure of the space of Willmore immersions. Because the conformal group of transformations of $\mathbb{R}^{m}$ is not compact, one cannot expect the space of Willmore immersions to be closed in the strong $C^{l}$-topology. However, locally, in isothermic coordinates, ${ }^{2}$ under some universal energy threshold, and as long as the conformal

\footnotetext{
${ }^{1}$ Minimal surfaces satisfy $\vec{H}=\overrightarrow{0}$ and are hence absolute minimizers of $W$.

${ }^{2}$ Analogously to other gauge-invariant problems, such as in Yang-Mills theory, isothermic coordinates (i.e., conformal parametrizations) provide the optimal symmetry-breaking method. A detailed discussion of this topic is available in [Rivière 2010].
} 
parameter $\lambda$ of the induced metric $g$ is controlled in $L^{\infty}$, the immersion is uniformly bounded in any $C^{l}$-norm. More precisely, the following $\varepsilon$-regularity result holds.

Theorem 1.1 [Rivière 2008]. There exists $\varepsilon_{0} \equiv \varepsilon_{0}(m)>0$ such that, for any Willmore conformal immersion $\vec{\Phi}: B_{1} \rightarrow \mathbb{R}^{m}$ satisfying

$$
\int_{B_{1}}|\nabla \vec{n}|^{2} d x<\varepsilon_{0}
$$

and for any $l \in \mathbb{N}^{*}$, we have

$$
\left\|\mathrm{e}^{-\lambda} \nabla^{l} \vec{\Phi}\right\|_{L^{\infty}\left(B_{1 / 2}\right)}^{2} \leq C_{l}\left(\int_{B_{1}}|\nabla \vec{n}|^{2} d x+1\right),
$$

where $C_{l}$ only depends on $l$, while $\lambda$ denotes the conformal parameter of $\vec{\Phi}$. Namely, $\lambda=\left\|\log \left|\partial_{x_{1}} \vec{\Phi}\right|\right\|_{L^{\infty}\left(B_{1}\right)}=\left\|\log \left|\partial_{x_{2}} \vec{\Phi}\right|\right\|_{L^{\infty}\left(B_{1}\right)}$.

This theorem leads to the concentration of compactness "dialectic" developed by Sacks and Uhlenbeck. In a conformal parametrization, assuming that the conformal factor is $L^{\infty}$-controlled in some subdomain of $\Sigma$, a sequence of Willmore immersions might fail to converge strongly in $C^{l}$ only at finitely many isolated points, namely, at those points where the $W^{1,2}$-norm of the Gauss map concentrates. Assuming their induced metric generates a sequence of conformal classes which remains within a compact subdomain of the moduli space of $\Sigma$, the control of the conformal factor of a sequence of conformal immersions with uniformly bounded Willmore energy is also guaranteed, except again at those isolated points. This fact is established in [Rivière 2013], and it ultimately follows from the works [Toro 1995; Müller and Šverák 1995; Hélein 1996] on immersions with totally bounded curvature.

In this context, it appears natural to consider a branched Willmore immersion and study its local behavior near the point singularities. ${ }^{3}$ In particular, we shall seek conditions that ensure the removability of the branch points.

In this paper, a branch point is a point where the immersion $\vec{\Phi}$ degenerates in the sense that $d \vec{\Phi}$ vanishes at that point. We focus on (conformal) locally Lipschitz and $W^{2,2}$ immersions $\vec{\Phi}: D^{2} \backslash\{0\} \rightarrow \mathbb{R}^{m}$ with a branch point at the origin 0 , and regular away from the origin. A priori, at a branch point, the mean curvature is singular. We will show that $\vec{\Phi}$, and thus the mean curvature, is actually smooth through a branch point, provided a certain set of sharp conditions are satisfied. The density $\theta_{0} \in \mathbb{N}^{*}$ of the current $\vec{\Phi}_{*}\left[D^{2}\right]$ is called the order of the branch point. We shall use the words branch point and singularity interchangeably. This is of course an abuse of language, as the immersion $\vec{\Phi}$ is not singular at a branch point. In

\footnotetext{
${ }^{3}$ Such point singularities also naturally occur as blow-ups of the Willmore flow.
} 
fact, both $\vec{\Phi}$ and $d \vec{\Phi}$ are well-defined there. It is the immersive nature of $\vec{\Phi}$ which degenerates at a branch point.

More generally, let $\Sigma$ be an open surface, and suppose that $f: \Sigma \rightarrow B_{R}^{m}(0) \backslash\{0\}$, for some $R>0$, is a smooth proper Willmore immersion. We define the associated two-varifold

$$
\mu:=\left[x \mapsto \mathscr{H}^{0}\left(f^{-1}(x)\right)\right] \mathscr{H}^{2}\llcorner f(\Sigma),
$$

and suppose that

$$
0 \in \operatorname{spt}(\mu), \quad \theta_{*}^{2}(\mu, 0)<\infty, \quad \int_{\Sigma}|\mathbb{0}|_{g}^{2} d \operatorname{vol}_{g}<\infty .
$$

It is shown in [Kuwert and Schätzle 2007] that $\theta^{2}(\mu, 0) \in \mathbb{N}$ exists, and that $\operatorname{spt}(\mu)$ is a smooth, possibly multivalued graph, over some planes in $B_{\rho}^{m}(0) \backslash B_{\rho / 2}^{m}(0)$ for some $\rho>0$. As we seek to understand the local behavior of our surface near the origin, we assume that there is exactly one graph of integer-multiplicity $\theta^{2}(\mu, 0) \geq \theta_{0} \geq 1$. We can then switch to the parametric formulation used above and throughout this paper. A celebrated inequality of [Li and Yau 1982] for varifolds with compact support gives

$$
\theta^{2}(\mu, 0):=\lim _{r \searrow 0} \frac{\mu\left(B_{r}^{m}(0)\right)}{\pi r^{2}} \leq \frac{1}{4 \pi} W(\Sigma) .
$$

Accordingly, studying surfaces with a high-order branch point amounts to doing away with hypotheses demanding low upper bounds on the Willmore energy (such as the assumption $W(\Sigma)<8 \pi$ in [Kuwert and Schätzle 2004]).

In the context of this paper, the word removability is to be understood with care. To say that a branch point is removable does not mean that it is the result of some "parametric illusion". Rather, it means that the map $\vec{\Phi}$ is smooth through the branch point, although it continues to fail to be an immersion at that point. In particular, the mean curvature, which is naturally singular at a branch point, turns out to be regular at a removable branch point. For instance, in this sense, the branched immersion $\vec{\Phi}: x \mapsto\left(x^{2}, x^{3}\right)$ has a removable branch point at the origin. In particular, the corresponding Gauss map which identifies to the $\mathbb{C P} \mathbb{P}^{1}$-projection of $\partial_{x} \vec{\Phi}: \vec{n}(x):=\left[2 x, 3 x^{2}\right] \equiv[2,3 x]$ is clearly smooth through the origin.

In [Bernard and Rivière 2011a], we delve deeper into the analysis of sequences of Willmore surfaces with uniformly bounded energy and nondegenerating conformal type. The results of the present paper play an important role there.

1B. Main results. Kuwert and Schätzle [2004] initiated the analytical study of point singularities of Willmore immersions by first considering unit-density singularities in codimension 1 . They were able to find some removability criterion (extended in [Rivière 2008] to arbitrary codimension). Unfortunately, the energy 
restrictions necessary to ensure that the singularities occurring have unit-density are quite stringent (namely, one must assume the immersion has Willmore energy strictly below $8 \pi$ ). Still in codimension 1, Kuwert and Schätzle [2007] studied singularities of higher order, thereby allowing less stringent bounds on the energy. This time, however, no removability condition was found.

In the present work, we bridge the gaps left by previous studies. We work in arbitrary codimension and impose no restriction on the Willmore energy bound (i.e., we allow the order of the branch point to be arbitrarily large — although finite). Even in this general setting, we are able to find point-removability conditions.

Working in arbitrary codimension goes beyond the mere technical prowess. Willmore surfaces immersed into $\mathbb{R}^{m}$ for $m>3$ are far from being devoid of interest, and the case $m=4$ is particularly useful in geometry, as seen in [Burstall et al. 2002; Ejiri 1988; Montiel 2000].

While in codimension 1 techniques have been developed and used, analytical results in higher codimension require a suitable reformulation of the problem. Briefly speaking, the codimension-1 case involves a fourth-order nonlinear scalar equation, whereas, in higher codimension, one faces a strongly coupled fourthorder nonlinear system of equations. For this reason, we adopt a radically different approach to the problem, by using the original framework devised in [Rivière 2008]. In particular, working in a conformal parametrization, the aforementioned system is recast into a single equation in divergence form for the mean curvature vector. The analytical efficiency and benefits of this method have come to fruition in [Rivière 2008; 2013; Bernard and Rivière 2011a; 2011b].

Our main goal is twofold. Firstly, we study the regularity of the Gauss map, and we develop precise asymptotics for the immersion and the mean curvature near that point. Secondly, we bring into light explicit conditions ensuring the removability of the point singularity.

We assume that the point singularity lies at the origin, and we localize the problem by considering a map $\vec{\Phi}: D^{2} \rightarrow \mathbb{R}^{m \geq 3}$, which is an immersion of $D^{2} \backslash\{0\}$, and satisfying

(i) $\vec{\Phi} \in C^{0}\left(D^{2}\right) \cap C^{\infty}\left(D^{2} \backslash\{0\}\right)$;

(ii) $\mathscr{H}^{2}\left(\vec{\Phi}\left(D^{2}\right)\right)<\infty$;

(iii) $\int_{D^{2}}|\overrightarrow{\mid}|_{g}^{2} d \operatorname{vol}_{g}<\infty$.

As explained in [Rivière 2010], under the above assumptions, using the moving frame method of Chern and Hélein, one can construct a Lipschitz diffeomorphism $f$ of the disk such that $\vec{\Phi} \circ f$ is conformal (an analogous procedure based on the work Müller and Sverak is presented in [Kuwert and Schätzle 2007]). We shall abusively continue to denote this reparametrization by $\vec{\Phi}$. It has properties (i)-(iii), 
and, moreover,

$$
\vec{\Phi}(0)=\overrightarrow{0} \quad \text { and } \quad \vec{\Phi}\left(D^{2}\right) \subset B_{R}^{m}(0) \quad \text { for some } 0<R<\infty .
$$

Hence, $\vec{\Phi} \in W_{\text {loc }}^{1, \infty} \cap W^{2,2}\left(D^{2} \backslash\{0\}\right)$. Away from the origin, we define the Gauss map $\vec{n}$ via

$$
\vec{n}=\star \frac{\partial_{x_{1}} \vec{\Phi} \wedge \partial_{x_{2}} \vec{\Phi}}{\left|\partial_{x_{1}} \vec{\Phi} \wedge \partial_{x_{2}} \vec{\Phi}\right|},
$$

where $\left\{x_{1}, x_{2}\right\}$ are standard Cartesian coordinates on the unit disk $D^{2}$, and $\star$ is the Euclidean Hodge-star operator. The immersion $\vec{\Phi}$ is conformal; i.e.,

$$
\left|\partial_{x_{1}} \vec{\Phi}\right|=\mathrm{e}^{\lambda}=\left|\partial_{x_{2}} \vec{\Phi}\right| \quad \text { and } \quad \partial_{x_{1}} \vec{\Phi} \cdot \partial_{x_{2}} \vec{\Phi}=0
$$

where $\lambda$ is the conformal parameter. An elementary computation shows that

$$
d \operatorname{vol}_{g}=\mathrm{e}^{2 \lambda} d x \quad \text { and } \quad|\nabla \vec{n}|^{2} d x=|d \vec{n}|_{g}^{2} d \operatorname{vol}_{g}=|\overrightarrow{\mathbb{d}}|_{g}^{2} d \operatorname{vol}_{g} .
$$

Hence, by hypothesis, we see that $\vec{n} \in W^{1,2}\left(D^{2} \backslash\{0\}\right)$. In dimension two, the 2-capacity of isolated points is null, so we actually have $\vec{n} \in W^{1,2}\left(D^{2}\right)$. Rescaling if necessary, we shall henceforth always assume that

$$
\int_{D^{2}}|\nabla \vec{n}|^{2} d x<\varepsilon_{0}
$$

where the adjustable parameter $\varepsilon_{0} \equiv \varepsilon_{0}(m)$ is chosen to fit our various needs (in particular, we will need it to be "small enough" in Proposition C.1).

For the sake of the following paragraph, we consider a conformal immersion $\vec{\Phi}$ : $D^{2} \rightarrow \mathbb{R}^{m}$, which is smooth across the unit disk. We introduce the local coordinates $\left(x_{1}, x_{2}\right)$ for the flat metric on the unit disk $D^{2}=\left\{x=\left(x_{1}, x_{2}\right) \in \mathbb{R}^{2}: x_{1}^{2}+x_{2}^{2}<1\right\}$. The operators $\nabla=\left(\partial_{x_{1}}, \partial_{x_{2}}\right), \nabla^{\perp}=\left(-\partial_{x_{2}}, \partial_{x_{1}}\right)$, div $=\nabla \cdot$, and $\Delta=\nabla \cdot \nabla$ will be understood in these coordinates. The conformal parameter $\lambda$ is defined as in (1-2). We set

$$
\vec{e}_{j}:=\mathrm{e}^{-\lambda} \partial_{x_{j}} \vec{\Phi} \quad \text { for } j \in\{1,2\} .
$$

As $\vec{\Phi}$ is conformal, $\left\{\vec{e}_{1}(x), \vec{e}_{2}(x)\right\}$ forms an orthonormal basis of the tangent space $T_{\vec{\Phi}(x)} \vec{\Phi}\left(D^{2}\right)$. Owing to the topology of $D^{2}$, there exists for almost every $x \in D^{2}$ a positively oriented orthonormal basis $\left\{\vec{n}_{1}, \ldots, \vec{n}_{m-2}\right\}$ of the normal space $N_{\vec{\Phi}(x)} \vec{\Phi}\left(D^{2}\right)$, such that $\left\{\vec{e}_{1}, \vec{e}_{2}, \vec{n}_{1}, \ldots, \vec{n}_{m-2}\right\}$ forms a basis of $T_{\vec{\Phi}(x)} \mathbb{R}^{m}$. From the Plücker embedding, realizing the Grassmannian $\mathrm{Gr}_{m-2}\left(\mathbb{R}^{m}\right)$ as a submanifold of the projective space of the $(m-2)$-th exterior power $\mathbb{P}\left(\bigwedge^{m-2} \mathbb{R}^{m}\right)$, we can represent the Gauss map as the $(m-2)$-vector $\vec{n}=\bigwedge_{\alpha=1}^{m-2} \vec{n}_{\alpha}$. Via the Hodge 
operator $\star$, we identify vectors and $(m-1)$-vectors in $\mathbb{R}^{m}$; namely,

$$
\star\left(\vec{n} \wedge \vec{e}_{1}\right)=\vec{e}_{2}, \quad \star\left(\vec{n} \wedge \vec{e}_{2}\right)=-\vec{e}_{1}, \quad \star\left(\vec{e}_{1} \wedge \vec{e}_{2}\right)=\vec{n} .
$$

In this notation, the second fundamental form $\vec{\mathbb{}}$, which is a symmetric 2-form on $T_{\vec{\Phi}(x)} \vec{\Phi}\left(D^{2}\right)$ into $N_{\vec{\Phi}(x)} \vec{\Phi}\left(D^{2}\right)$, is expressed as

$$
\vec{\mathbb{}}=\sum_{\alpha, i, j} \mathrm{e}^{-2 \lambda} h_{i j}^{\alpha} \vec{n}_{\alpha} d x_{i} \otimes d x_{j} \equiv \sum_{\alpha, i, j} h_{i j}^{\alpha} \vec{n}_{\alpha}\left(\vec{e}_{i}\right)^{*} \otimes\left(\vec{e}_{j}\right)^{*}
$$

where

$$
h_{i j}^{\alpha}=-\mathrm{e}^{-\lambda} \vec{e}_{i} \cdot \partial_{x_{j}} \vec{n}_{\alpha}
$$

The mean curvature vector is

$$
\vec{H}=\sum_{\alpha=1}^{m-2} H^{\alpha} \vec{n}_{\alpha}=\frac{1}{2} \sum_{\alpha=1}^{m-2}\left(h_{11}^{\alpha}+h_{22}^{\alpha}\right) \vec{n}_{\alpha} .
$$

The Willmore equation [Weiner 1978] is cast in the form

$$
\Delta_{\perp} \vec{H}+\sum_{\alpha, \beta, i, j} h_{i j}^{\alpha} h_{i j}^{\beta} H^{\beta} \vec{n}_{\alpha}-2|\vec{H}|^{2} \vec{H}=0,
$$

with

$$
\Delta_{\perp} \vec{H}:=\mathrm{e}^{-2 \lambda} \pi_{\vec{n}} \operatorname{div}\left(\pi_{\vec{n}}(\nabla \vec{H})\right),
$$

and $\pi_{\vec{n}}$ is the projection onto the normal space spanned by $\left\{\vec{n}_{\alpha}\right\}_{\alpha=1}^{m-2}$.

The Willmore equation (1-6) is a fourth-order nonlinear equation (in the coefficients of the induced metric, which depends on $\vec{\Phi}$ ). With respect to the coefficients $H^{\alpha}$ of the mean curvature vector, it is actually a strongly coupled nonlinear system whose study is particularly challenging. In codimension 1, there is one equation for the scalar curvature; in higher codimension, however, the situation becomes significantly more complicated, and one must seek different techniques to approach the problem. Fortunately, in a conformal parametrization, it is possible ${ }^{4}$ to recast the system (1-6) in an equivalent, yet analytically more suitable, form [Rivière 2008]. Namely, we have ${ }^{5}$

$$
\operatorname{div}\left(\nabla \vec{H}-3 \pi_{\vec{n}}(\nabla \vec{H})+\star\left(\nabla^{\perp} \vec{n} \wedge \vec{H}\right)\right)=0 .
$$

This remarkable reformulation in divergence form of the Willmore equation is the starting point of our analysis. In our singular situation, (1-7) holds only away from

\footnotetext{
${ }^{4}$ This procedure requires choosing the normal frame $\left\{\vec{n}_{\alpha}\right\}$ astutely. See [Rivière 2008] for details.

${ }^{5}$ The operators $\nabla:=\left(\partial_{x_{1}}, \partial_{x_{2}}\right), \nabla^{\perp}:=\left(-\partial_{x_{2}}, \partial_{x_{1}}\right)$, and div $:=\nabla \cdot$ are understood with respect to flat coordinates $\left\{x_{1}, x_{2}\right\}$ on the unit disk.
} 
the origin, on $D^{2} \backslash\{0\}$. In particular, we can define the constant $\vec{\gamma}_{0} \in \mathbb{R}^{m}$, called first residue, by

$$
\vec{\gamma}_{0}:=\frac{1}{4 \pi} \int_{\partial D^{2}} \vec{v} \cdot\left(\nabla \vec{H}-3 \pi_{\vec{n}}(\nabla \vec{H})+\star\left(\nabla^{\perp} \vec{n} \wedge \vec{H}\right)\right),
$$

where $\vec{v}$ denotes the unit outward normal vector to $\partial D^{2}$. We will see in Corollary 1.5 that the residue appears in the local asymptotic expansion of the mean curvature vector around the singularity. The residue $\vec{\gamma}_{0}$ as expressed in (1-8) already appears in [Rivière 2008], where the second author studies Willmore immersions with a unit-density point singularity, thereby generalizing in arbitrary codimension the results of [Kuwert and Schätzle 2004]. Although in the end identical to $\vec{\gamma}_{0}$, the residue used in the latter is defined differently.

We next state a result describing the regularity of the Gauss map around the point singularity. ${ }^{6}$

Proposition 1.2. Let $\vec{\Phi} \in C^{\infty}\left(D^{2} \backslash\{0\}\right) \cap\left(W^{2,2} \cap W^{1, \infty}\right)\left(D^{2}\right)$ be a conformal Willmore immersion of the punctured disk into $\mathbb{R}^{m}$ whose Gauss map $\vec{n}$ lies in $W^{1,2}\left(D^{2}\right)$. Then $\nabla^{2} \vec{n} \in L^{2, \infty}\left(D^{2}\right)$, and thus in particular $\nabla \vec{n}$ is an element of BMO. Furthermore, $\vec{n}$ satisfies the pointwise estimate

$$
|\nabla \vec{n}(x)| \lesssim|x|^{-\epsilon} \quad \text { for all } \epsilon>0 .
$$

If the order of degeneracy of the immersion $\vec{\Phi}$ at the origin is at least two, ${ }^{7}$ then $\nabla \vec{n}$ belongs to $L^{\infty}\left(B_{1}(0)\right)$.

A conformal immersion of $D^{2} \backslash\{0\}$ into $\mathbb{R}^{m}$ such that $\nabla \vec{\Phi}$ and the Gauss map $\vec{n}$ both extend to maps in $W^{1,2}\left(D^{2}\right)$ has a distinctive behavior near the point singularity located at the origin. One shows (see [Müller and Šverák 1995] and Lemma A.5 in [Rivière 2013]) that there exists a positive integer $\theta_{0}$ with

$$
|\vec{\Phi}(x)| \simeq|x|^{\theta_{0}} \text { and } \quad|\nabla \vec{\Phi}(x)| \simeq|x|^{\theta_{0}-1} \quad \text { near the origin. }
$$

In addition, we have

$$
\lambda(x):=\frac{1}{2} \log \left(\frac{1}{2}|\nabla \vec{\Phi}(x)|^{2}\right)=\left(\theta_{0}-1\right) \log |x|+u(x),
$$

where $u \in W^{2,1}\left(D^{2}\right)$, and one has

$$
\begin{cases}\nabla \lambda \in L^{2}\left(D^{2}\right) & \text { when } \theta_{0}=1 \\ |\nabla \lambda(x)| \lesssim|x|^{-1} \in L^{2, \infty}\left(D^{2}\right) & \text { when } \theta_{0} \geq 2\end{cases}
$$

The function $\mathrm{e}^{-u(x)} \equiv|x|^{\theta_{0}-1} \mathrm{e}^{-\lambda(x)}$ is continuous and strictly positive in a small neighborhood of the origin.

\footnotetext{
${ }^{6}$ In codimension 1, the statement of Proposition 1.2 was the object of [Kuwert and Schätzle 2007].

${ }^{7}$ Roughly speaking, if $\nabla \vec{\Phi}(0)=\overrightarrow{0}$. The notion of "order of degeneracy" is made precise below.
} 
The integer $\theta_{0}$ is the density of the current $\vec{\Phi}_{*}\left[D^{2}\right]$ at the image point $0 \in \mathbb{R}^{m}$.

When such a conformal immersion is Willmore on $D^{2} \backslash\{0\}$, it is possible to refine the asymptotics (1-9). The following result describes the behavior of the immersion $\vec{\Phi}$ locally around the singularity at the origin.

Proposition 1.3. Let $\vec{\Phi}$ be as in Proposition 1.2 with conformal parameter $\lambda$, and let $\theta_{0}$ be as in (1-9). There exists a constant vector $\vec{A}=\vec{A}^{1}+i \vec{A}^{2} \in \mathbb{R}^{2} \otimes \mathbb{R}^{m}$ satisfying the following conditions:

(i) $\vec{A}^{1} \cdot \vec{A}^{2}=0, \quad\left|\vec{A}^{1}\right|=\left|\vec{A}^{2}\right|=\theta_{0}^{-1} \lim _{x \rightarrow 0} \frac{e^{\lambda(x)}}{|x|^{\theta_{0}-1}}, \quad \pi_{\vec{n}(0)} \vec{A}=\overrightarrow{0}$.

(ii) When $\theta_{0}=1$,

$$
\vec{\Phi}(x)=\Re(\vec{A} x)+\vec{\zeta}(x),
$$

$$
\begin{aligned}
& \text { with } \vec{\zeta} \in \bigcap_{p<\infty} W^{2, p}\left(D^{2}\right) \text { and } \\
& \quad \vec{\zeta}(x)=\mathrm{O}\left(|x|^{2-\epsilon}\right), \quad \nabla \vec{\zeta}(x)=\mathrm{O}\left(|x|^{1-\epsilon}\right) \quad \text { for all } \epsilon>0 .
\end{aligned}
$$

(iii) When $\theta_{0} \geq 2$,

$$
\vec{\Phi}(x)=\Re\left(\vec{A} x^{\theta_{0}}+\vec{B}_{1} x^{\theta_{0}+1}+\vec{C}_{\theta_{0}-1}|x|^{2 \theta_{0}} x^{1-\theta_{0}}\right)+|x|^{\theta_{0}-1} \vec{\xi}(x),
$$

where $\vec{B}_{1}$ and $\vec{C}_{\theta_{0}-1}$ are constant vectors in $\mathbb{C}^{m}$, and moreover for all $\epsilon>0$,

$$
\vec{\xi}(x)=\mathrm{O}\left(|x|^{3-\epsilon}\right), \quad \nabla \vec{\xi}(x)=\mathrm{O}\left(|x|^{2-\epsilon}\right), \quad \nabla^{2} \vec{\xi}(x)=\mathrm{O}\left(|x|^{1-\epsilon}\right) .
$$

The plane $\operatorname{span}\left\{\vec{A}^{1}, \vec{A}^{2}\right\}$ is tangent to the surface at the origin. If $\theta_{0}=1$, this plane is actually $T_{0} \Sigma$. One can indeed show that the tangent unit vectors $\vec{e}_{j}(0)$ spanning $T_{0} \Sigma$ (defined in (1-5)) satisfy $\vec{e}_{j}(0)=\vec{A}^{j} /\left|\vec{A}^{j}\right|$. In contrast, when $\theta_{0} \geq 2$, the tangent plane $T_{0} \Sigma$ does not exist in the classical sense, and the vectors $\vec{e}_{j}(x)$ "spin" as $x$ approaches the origin (see (2-21)). More precisely, $T_{0} \Sigma$ is the plane $\operatorname{span}\left\{\vec{A}^{1}, \vec{A}^{2}\right\}$ covered $\theta_{0}$ times.

Remark 1.4. When $\theta_{0}=1$, the immersion $\vec{\Phi}$ belongs to $C^{1, \alpha}\left(D^{2}\right)$ for all $\alpha \in[0,1)$. In general however, $\vec{\Phi}$ need not be $C^{1,1}\left(D^{2}\right)$. To see this, it suffices to invert the standard catenoid ${ }^{8}$ about the origin, thereby yielding a Willmore surface ${ }^{9}$ which comprises near the origin two identical graphs (mirror-symmetric), each degenerating with order $\theta_{0}=1$ at the origin. One then directly verifies that

$$
|\nabla \vec{n}(x)| \simeq-\log |x| \in \mathrm{BMO} \backslash L^{\infty}\left(D^{2}\right) .
$$

Hence, we cannot expect in general $\epsilon=0$ in (1-11). Moreover, $\vec{\Phi} \notin C^{1,1}\left(D^{2}\right)$.

\footnotetext{
${ }^{8}$ Conformally parametrized by $(r, \varphi) \mapsto\left(\left(r+r^{-1}\right) \cos (\varphi),\left(r+r^{-1}\right) \sin (\varphi),-2 \log (r)\right)$.

${ }^{9}$ For it is the image of a minimal (thus Willmore) surface under a Möbius transformation.
} 
One also verifies that the inverted catenoid in $\mathbb{R}^{3}$ and centered around the $(0,0,1)$ axis has first residue $\vec{\gamma}_{0}=-4(0,0,1)$.

Having obtained the asymptotic behavior of the immersion $\vec{\Phi}$ and its first two derivatives near the origin, it is possible to obtain analogous information for the mean curvature vector. This is the object of the next proposition.

Corollary 1.5. Let $\vec{\Phi}$ be as in Proposition $1.2, \lambda$ be its conformal parameter, and $\theta_{0}$ be as in (1-9). Locally around the singularity, the mean curvature vector satisfies

(i) when $\theta_{0}=1$,

$$
\vec{H}(x)+\vec{\gamma}_{0} \log |x| \in \bigcap_{p<\infty} W^{1, p}\left(D^{2}\right),
$$

where $\vec{\gamma}_{0}$ is the residue defined in (1-8);

(ii) when $\theta_{0} \geq 2$,

$$
e^{\lambda(x)} \vec{H}(x)=2 \theta_{0} \mathrm{e}^{-u(x)} \mathfrak{R}\left[\vec{C}_{\theta_{0}-1}\left(\frac{|x|}{x}\right)^{\theta_{0}-1}\right]+\mathrm{O}\left(|x|^{1-\epsilon}\right) \quad \text { for all } \epsilon>0,
$$

where $\vec{C}_{\theta_{0}-1} \in \mathbb{C}^{m}$ is the same constant vector as in Proposition 1.3(iii), and

$$
\mathrm{e}^{u(x)}:=|x|^{1-\theta_{0}} \mathrm{e}^{\lambda(x)} \in C^{0}\left(D^{2},(0, \infty)\right) .
$$

In particular, since $\vec{H}$ is a normal vector, we note that $\pi_{\vec{n}(0)} \vec{C}_{\theta_{0}-1}=\vec{C}_{\theta_{0}-1}$.

When $\theta_{0} \geq 2$, the weighted mean curvature vector $\mathrm{e}^{\lambda} \vec{H}$ is thus bounded across the singularity (unlike in the case $\theta_{0}=1$, where it behaves logarithmically). But its limit may not exist: $\mathrm{e}^{\lambda(x)} \vec{H}(x)$ is a "spinning vector" as $x$ approaches the origin. ${ }^{10}$ We may recast the expansion given in Corollary 1.5(ii) in the form

$$
\vec{H}(x)=2 \theta_{0} \mathrm{e}^{-2 u(0)} \mathfrak{R}\left(\vec{C}_{\theta_{0}-1} x^{1-\theta_{0}}\right)+\mathrm{O}\left(|x|^{2-\theta_{0}-\epsilon}\right) .
$$

In this formulation, $\vec{H}$ appears as the sum of an harmonic function with a pole at the origin of order $\left(\theta_{0}-1\right)$ and of a rest of lower order. This feature persists even when $\vec{C}_{\theta_{0}-1}=\overrightarrow{0}$ and it can be precisely quantified, namely:

Proposition 1.6. Let $\vec{\Phi}$ be as in Proposition 1.2, $\lambda$ be its conformal parameter, and $\theta_{0}$ be as in (1-9). There exists a complex-valued function $\vec{T}$ satisfying

$$
\partial_{x} \vec{T}=\mathrm{O}(|\vec{H}||\nabla \vec{n}|) \quad \text { and } \quad \vec{T}=\mathrm{O}\left(|x|^{2-\theta_{0}-\epsilon}\right) \quad \text { for all } \epsilon>0,
$$

and such that, locally around the singularity, the mean curvature vector satisfies

$$
\vec{H}(x)+\vec{\gamma}_{0} \log |x|=\Re(\vec{E}(x)-\vec{T}(x)),
$$

\footnotetext{
${ }^{10}$ But the function $\mathrm{e}^{-u(x)}$ does have a finite, positive limit at $x=0$, as shown in [Müller and Šverák 1995].
} 
where $\vec{\gamma}_{0}$ is the residue defined in (1-8). The function $\vec{E}$ is antiholomorphic with possibly a pole at the origin of order at most $\left(\theta_{0}-1\right)$.

If the singularity of $\vec{E}$ at the origin has order $\alpha \in\left\{0, \ldots, \theta_{0}-1\right\}$, then the functions $\vec{E}$ and $\vec{T}$ can be adjusted to satisfy

$$
\vec{E}-\vec{T}=\vec{E}_{\alpha} \bar{x}^{-\alpha}-\vec{Q}
$$

for some nonzero constant vector $\vec{E}_{\alpha} \in \mathbb{C}^{m}$, and with

$$
\partial_{x} \vec{Q}=\mathrm{O}(|\vec{H}||\nabla \vec{n}|) \quad \text { and } \quad \vec{Q}=\mathrm{O}\left(|x|^{1-\alpha-\epsilon}\right) \quad \text { for all } \epsilon>0 .
$$

Here $\bar{x}$ denotes the complex conjugate of $x \in \mathbb{R}^{2} \simeq \mathbb{C}$.

We may view the function $\vec{E}$ from the previous proposition as a string of $m$ complex-valued functions $\left\{E_{j}\right\}_{j=1, \ldots, m}$, each of which is antiholomorphic and possibly has a pole at the origin of order at most $\left(\theta_{0}-1\right)$. This prompts us to introduce the following decisive quantity.

Definition 1.7. The second residue associated with the immersion $\vec{\Phi}$ at the origin is the $\mathbb{N}^{m}$-valued vector

$$
\vec{\gamma}=\left(\gamma_{1}, \ldots, \gamma_{m}\right) \quad \text { with } \gamma_{j}:=\frac{1}{2 i \pi} \int_{\partial D^{2}} d \log E_{j} \in \mathbb{N} .
$$

The importance of $\vec{\gamma}$ cannot be overstated: it controls the leading-order singular behavior of the mean curvature at the origin, as the following statement shows.

Theorem 1.8. Let $\vec{\Phi}$ be as in Proposition 1.2 and let $\lambda$ be its conformal parameter, $\theta_{0}$ as in (1-9), and the residues $\vec{\gamma}_{0}$ and $\vec{\gamma}$ as in (1-8) and (1-14), respectively. Define

$$
\alpha:=\max _{1 \leq j \leq m} \gamma_{j} \in\left\{0, \ldots, \theta_{0}-1\right\} .
$$

Then $\nabla^{\theta_{0}+1-\alpha} \vec{n} \in L^{2, \infty}\left(D^{2}\right)$, and thus $\nabla^{\theta_{0}-\alpha} \vec{n} \in \operatorname{BMO}\left(D^{2}\right)$.

Locally around the origin, the immersion has the asymptotic expansion $\vec{\Phi}(x)=\Re\left(\vec{A} x^{\theta_{0}}+\sum_{j=1}^{\theta_{0}-\alpha} \vec{B}_{j} x^{\theta_{0}+j}+\vec{C}_{\alpha}|x|^{2 \theta_{0}} x^{-\alpha}\right)-\vec{C}|x|^{2 \theta_{0}}\left(\log |x|^{2 \theta_{0}}-4\right)+\vec{\xi}(x)$, where $\vec{B}_{j}$ and $\vec{C}_{\alpha} \in \mathbb{C}^{m}$ are constant vectors, $\vec{A}$ is as in Proposition 1.3, and ${ }^{11}$ $\vec{C}:=\mathrm{e}^{2 u(0)} /\left(2 \theta_{0}^{3}\right) \vec{\gamma}_{0}$. Furthermore, the function $\vec{\xi}$ satisfies the estimates

$$
\begin{gathered}
\nabla^{j} \vec{\xi}(x)=\mathrm{O}\left(|x|^{2 \theta_{0}-\alpha-j+1-\epsilon}\right) \quad \text { for all } \epsilon>0 \text { and } j \in\left\{0, \ldots, \theta_{0}-\alpha+1\right\}, \\
|x|^{1-\theta_{0}} \nabla^{\theta_{0}-\alpha+2} \vec{\xi} \in \bigcap_{p<\infty} L^{p}
\end{gathered}
$$

${ }^{11}$ The function $u$ is as in Corollary 1.5 . 
In particular, we have

$$
\vec{H}(x)=\Re\left(\vec{E}_{\alpha} \bar{x}^{-\alpha}\right)-\vec{\gamma}_{0} \log |x|+\vec{\eta}(x),
$$

where $\vec{E}_{\alpha}:=2 \theta_{0}\left(\theta_{0}-\alpha\right) \mathrm{e}^{-2 u(0)} \vec{C}_{\alpha}^{*}$. The function $\vec{\eta}$ satisfies

$$
\begin{gathered}
\nabla^{j} \vec{\eta}(x)=\mathrm{O}\left(|x|^{1-j-\alpha-\epsilon}\right) \quad \text { for all } \epsilon>0 \text { and } j \in\left\{0, \ldots, \theta_{0}-\alpha-1\right\}, \\
\qquad|x|^{\theta_{0}-1} \nabla^{\theta_{0}-\alpha} \vec{\eta} \in \bigcap_{p<\infty} L^{p} .
\end{gathered}
$$

From this result, it comes as no surprise to discover that the simultaneous vanishing of both residues $\vec{\gamma}_{0}$ and $\vec{\gamma}$ improves the regularity of the immersion $\vec{\Phi}$. This is the content of the next statement.

Theorem 1.9. Under the hypotheses of Corollary 1.5, suppose that the first residue vanishes: $\vec{\gamma}_{0}=\overrightarrow{0}$.

(i) When $\theta_{0}=1$, the immersion $\vec{\Phi}$ is smooth across the branch point.

(ii) When $\theta_{0}>1$, if in addition the second residue vanishes $\vec{\gamma}=\overrightarrow{0}$, the immersion $\vec{\Phi}$ is smooth across the branch point.

Remark 1.10. Let $\vec{\Phi}: D^{2} \rightarrow \mathbb{R}^{m \geq 3}$ be a minimal immersion with a branch point at the origin. Since minimal immersions have vanishing first and second residues (see Remark 2.6), Theorem 1.9 applies and singularities are removable.

We close this section with an important observation. When the Willmore immersion $\vec{\Phi}$ is smooth, the Willmore equation written in divergence form (1-7) holds on the whole unit disk $D^{2}$. Hence, the first residue $\vec{\gamma}_{0}$ defined in (1-8) vanishes about every point. In turn, the expansion (1-13) given in Proposition 1.6 shows that the antiholomorphic function $\vec{E}$ cannot be singular anywhere in $D^{2}$, and thus in particular that the second residue $\vec{\gamma}$ also vanishes about every point.

Given a branched Willmore immersion, we have found some explicit conditions ensuring the removability of the branch points. These conditions require that certain residues vanish. Naturally, if the immersion is explicitly given, one may directly verify smoothness at the bad points, without resorting to the residues. This is of course not the situation which we have in mind. Suppose now that a sequence of smooth Willmore immersions is given, with uniformly $L^{2}$-bounded second fundamental form (the uniform bound need not be smaller than $8 \pi$ ). If the sequence does not degenerate in moduli space, ${ }^{12}$ it is known that one can extract a subsequence which converges strongly away from finitely many points to a limitimmersion which is Willmore and smooth away from finitely many isolated branch points. As residues are computed as circulation integrals along circles enlacing the singularities, one expects that they pass to the weak limit. This is indeed the case

\footnotetext{
${ }^{12}$ See [Bernard and Rivière 2011a] for further information on this condition.
} 
for the first residue $\vec{\gamma}_{0}$ (as one easily verifies), but it remains an open problem for the second residue $\vec{\gamma}$. Because each immersion is smooth, its residues vanish about every point. If one knew that the second residue passed through weak limits, one could then conclude that the residues associated with the limit-immersion vanished as well, thereby making the limit into a branched smooth Willmore immersion.

1C. Some examples. Covering three times over the inverted catenoid of Remark 1.4 gives rise to a conformal Willmore immersion which degenerates at the origin with order $\theta_{0}=3$. Yet, the geometry of the image is identical to that of the inverted catenoid, i.e., with a degeneracy of order 1 . This is an instance of a "false" thirdorder branch point simply resulting from having chosen a "bad" parametrization: the singularity truly has order one. Without surprise, in this case, we find that the first residue is $\overrightarrow{\gamma_{0}}=-12(0,0,1)$ (i.e., three times that of the singly covered inverted catenoid of Remark 1.4). The second residue $\vec{\gamma}$ vanishes.

A conformal parametrization of the 3-Enneper (minimal) surface in $\mathbb{R}^{3}$ is given by

$$
(r, \varphi) \mapsto\left(\frac{1}{3 r^{3}} \cos 3 \varphi-\frac{1}{r} \cos \varphi, \frac{1}{3 r^{3}} \sin 3 \varphi+\frac{1}{r} \sin \varphi, \frac{1}{r^{2}} \cos 2 \varphi-1\right) .
$$

Inverting this surface about the point $(0,0,0)$ gives rise to a compact Willmore surface whose conformal parametrization near $r=0$ satisfies

$$
\vec{\Phi}(r, \varphi)=3\left(r^{3} \cos 3 \varphi, r^{3} \sin 3 \varphi, 3 r^{4} \cos 2 \varphi\right)+\mathrm{O}\left(r^{5}, r^{5}, r^{6}\right) .
$$

This surface has a branch point of order $\theta_{0}=3$ at the origin, where the mean curvature is

$$
\vec{H}(r, \varphi)=\left(0,0, \frac{2}{3 r^{2}} \cos 2 \varphi\right)+\mathrm{O}\left(r^{-1}\right)
$$

The first residue is computed to be $\vec{\gamma}_{0}=\overrightarrow{0}$, and the second residue is $\vec{\gamma}=(0,0,2)$.

In codimension two, we consider now the following conformal parametrization of a minimal surface:

$$
(r, \varphi) \mapsto\left(\frac{1}{r^{3}} \cos 3 \varphi, \frac{1}{r^{3}} \sin 3 \varphi, \frac{1}{r} \cos \varphi, \frac{1}{r} \sin \varphi-1\right) .
$$

Inverting this surface about the origin in $\mathbb{R}^{4}$ gives rise to a compact Willmore surface whose conformal parametrization near $r=0$ satisfies

$$
\vec{\Phi}(r, \varphi)=\left(r^{3} \cos 3 \varphi, r^{3} \sin 3 \varphi, r^{5} \cos \varphi, r^{5} \sin \varphi\right)+\mathrm{O}\left(r^{7}, r^{7}, r^{9}, r^{6}\right),
$$

having too a branch point of order $\theta_{0}=3$ at the origin. The mean curvature is

$$
\vec{H}(r, \varphi)=\left(0,0, \frac{4}{r} \cos \varphi, \frac{4}{r} \sin \varphi\right)+\mathrm{O}(1)
$$


The second residue is $\vec{\gamma}=(0,0,1,1)$, while the first residue vanishes.

\section{Proofs of the theorems}

2A. Fundamental results and reformulation. We place ourselves in the situation described in Section 1B. Namely, we have a Willmore immersion $\vec{\Phi}$ on the punctured disk which degenerates at the origin in such a way that

$$
|\vec{\Phi}(x)| \simeq|x|^{\theta_{0}} \quad \text { and } \quad|\nabla \vec{\Phi}(x)|=\sqrt{2} \mathrm{e}^{\lambda(x)} \simeq|x|^{\theta_{0}-1}
$$

for some $\theta_{0} \in \mathbb{N} \backslash\{0\}$.

Amongst the analytical tools available to the study of weak Willmore immersions with square-integrable second fundamental form, an important one is certainly the $\varepsilon$-regularity. The version appearing in Theorem 2.10 and Remark 2.11 of [Kuwert and Schätzle 2001] (see also Theorem I.5 in [Rivière 2008]) states that there exists $\varepsilon_{0}>0$ such that, if

$$
\int_{B_{1}(0)}|\nabla \vec{n}|^{2} d x<\varepsilon_{0}
$$

then we have

$$
\left\|\mathrm{e}^{-\lambda} \nabla \vec{n}\right\|_{L^{\infty}\left(B_{\sigma}^{g}\right)} \leq \frac{C}{\sigma}\|\nabla \vec{n}\|_{L^{2}\left(B_{2 \sigma}^{g}\right)} \quad \text { for all } B_{2 \sigma}^{g} \subseteq \Omega:=D^{2} \backslash\{0\},
$$

where $B_{\sigma}^{g}$ is a geodesic disk of radius $\sigma$ for the induced metric $g=\vec{\Phi}^{*} g_{\mathbb{R}^{m}}$, and $C$ is a universal constant. As always, $\lambda$ denotes the conformal parameter.

The $\varepsilon$-regularity enables us to obtain the following result, already observed in [Kuwert and Schätzle 2007], and decisive to the remainder of the argument.

Lemma 2.1. The function $\delta(r):=r \sup _{|x|=r}|\nabla \vec{n}(x)|$ satisfies

$$
\lim _{r \searrow 0} \delta(r)=0 \quad \text { and } \quad \int_{0}^{1} \delta^{2}(r) \frac{d r}{r}<\infty
$$

Proof. From (1-3) and (1-9), the metric $g$ satisfies

$$
g_{i j}(x) \simeq|x|^{2\left(\theta_{0}-1\right)} \delta_{i j} \quad \text { on } B_{2 r}(0) \backslash B_{r / 2}(0) \text { for all } r \in(0,1 / 2) .
$$

A simple computation then shows that

$$
B_{2 c r^{\theta_{0}}}^{g}(x) \subset B_{2 r}(0) \backslash B_{r / 2}(0) \text { for all } x \in \partial B_{r}(0),
$$

where $0<2 \theta_{0} c<1-2^{-\theta_{0}}$. 
Since the metric $g$ does not degenerate away from the origin, given $0<r<1 / 2$, we can always cover the flat circle $\partial B_{r}(0)$ with finitely many metric disks:

$$
\partial B_{r}(0) \subset \bigcup_{j=1}^{N} B_{c r}^{g} \theta_{0}\left(x_{j}\right) \quad \text { with } x_{j} \in \partial B_{r}(0) .
$$

Hence, per the latter, (2-2), and (2-3), we obtain that for some $x_{0} \in \partial B_{r}(0)$ we have

$$
\begin{aligned}
r \sup _{|x|=r}|\nabla \vec{n}(x)| & \simeq r^{\theta_{0}} \sup _{|x|=r}\left|\mathrm{e}^{-\lambda(x)} \nabla \vec{n}(x)\right| \leq r^{\theta_{0}}\left\|\mathrm{e}^{-\lambda} \nabla \vec{n}\right\|_{L^{\infty}\left(B_{c r}^{g} \theta_{0}\left(x_{0}\right)\right)} \\
& \lesssim\|\nabla \vec{n}\|_{L^{2}\left(B_{2 c r}^{g} \theta_{0}\left(x_{0}\right)\right)} \leq\|\nabla \vec{n}\|_{L^{2}\left(B_{2 r}(0) \backslash B_{r / 2}(0)\right)} .
\end{aligned}
$$

As $\nabla \vec{n}$ is square-integrable by hypothesis, letting $r$ tend to zero in the latter yields the first assertion.

The second assertion follows from (2-4), namely,

$$
\int_{0}^{1 / 2} \delta^{2}(r) \frac{d r}{r} \lesssim \int_{0}^{1 / 2}\|\nabla \vec{n}\|_{L^{2}\left(B_{2 r}(0) \backslash B_{r / 2}(0)\right)}^{2} \frac{d r}{r}=\log (4)\|\nabla \vec{n}\|_{L^{2}\left(B_{1}(0)\right)}^{2},
$$

which is by hypothesis finite.

Recalling (1-3) linking the Gauss map to the mean curvature vector and the fact that $\mathrm{e}^{\lambda(x)} \simeq|x|^{\theta_{0}-1}$, we obtain from Lemma 2.1 that

$$
r^{\theta_{0}} \sup _{|x|=r}|\vec{H}(x)| \leq r^{\theta_{0}} \sup _{|x|=r} \mathrm{e}^{-\lambda(x)}|\nabla \vec{n}(x)| \lesssim \delta(r) .
$$

The Willmore equation (1-7) may be alternatively written

$$
\operatorname{div}\left(\nabla \vec{H}-3 \pi_{\vec{n}}(\nabla \vec{H})-\star\left(\vec{n} \wedge \nabla^{\perp} \vec{H}\right)\right)=0 \quad \text { on } \Omega:=B_{1}(0) \backslash\{0\} .
$$

It is elliptic [Rivière 2008]. Using the information on the gradient of $\vec{n}$ given by (2-2), and some standard analytical techniques for second-order elliptic equations in divergence form (see [Grüter and Widman 1982]), one deduces from (2-5) that

$$
r^{\theta_{0}+1} \sup _{|x|=r}|\nabla \vec{H}(x)| \lesssim \delta(r) .
$$

These observations shall be helpful in the sequel.

Equation (1-7) implies that, for any ball $B_{\rho}(0)$ of radius $\rho$ centered on the origin and contained in $\Omega$, we have

$$
\int_{\partial B_{\rho}(0)} \vec{v} \cdot\left(\nabla \vec{H}-3 \pi_{\vec{n}}(\nabla \vec{H})+\star\left(\nabla^{\perp} \vec{n} \wedge \vec{H}\right)\right)=4 \pi \vec{\gamma}_{0} \quad \text { for all } \rho \in(0,1),
$$

where $\vec{\gamma}_{0}$ is the residue defined in (1-8). Here $\vec{v}$ denotes the unit outward normal 
vector to $\partial B_{\rho}(0)$. An elementary computation shows that

$$
\int_{\partial B_{\rho}(0)} \vec{v} \cdot \nabla \log |x|=2 \pi \quad \text { for all } \rho>0 .
$$

Thus, upon setting

$$
\vec{X}:=\nabla \vec{H}-3 \pi_{\vec{n}}(\nabla \vec{H})+\star\left(\nabla^{\perp} \vec{n} \wedge \vec{H}\right)-2 \vec{\gamma}_{0} \nabla \log |x|,
$$

we find

$$
\operatorname{div} \vec{X}=0 \quad \text { on } \Omega
$$

and

$$
\int_{\partial B_{\rho}(0)} \vec{v} \cdot \vec{X}=0 \quad \text { for all } \rho \in(0,1)
$$

As $\vec{X}$ is smooth away from the origin, the Poincare lemma implies now the existence of an element $\vec{L} \in C^{\infty}(\Omega)$, defined up to an additive constant, such that

$$
\vec{X}=\nabla^{\perp} \vec{L} \quad \text { on } \Omega .
$$

We deduce from Lemma 2.1 and (2-5)-(2-9) that

$$
\int_{B_{1}(0)}|x|^{2 \theta_{0}}|\nabla \vec{L}|^{2} d x \lesssim \int_{0}^{1} \delta^{2}(s) \frac{d s}{s}<\infty .
$$

A classical Hardy-Sobolev inequality gives the estimate

$$
\theta_{0}^{2} \int_{B_{1}(0)}|x|^{2\left(\theta_{0}-1\right)}|\vec{L}|^{2} d x \leq \int_{B_{1}(0)}|x|^{2 \theta_{0}}|\nabla \vec{L}|^{2} d x+\theta_{0} \int_{\partial B_{1}(0)}|\vec{L}|^{2}
$$

which is a finite quantity, owing to (2-10) and to the smoothness of $\vec{L}$ away from the origin. The immersion $\vec{\Phi}$ has near the origin the asymptotic behavior

$$
|\nabla \vec{\Phi}(x)| \simeq|x|^{\theta_{0}-1} .
$$

Hence (2-11) yields that

$$
\vec{L} \cdot \nabla \vec{\Phi}, \vec{L} \wedge \nabla \vec{\Phi} \in L^{2}\left(B_{1}(0)\right) .
$$

We next set $\vec{F}(x):=2 \vec{\gamma}_{0} \log |x|$, and define the functions $g$ and $\vec{G}$ via

$$
\left\{\begin{aligned}
\Delta g & =\nabla \vec{F} \cdot \nabla \vec{\Phi}, & \Delta \vec{G} & =\nabla \vec{F} \wedge \nabla \vec{\Phi} & & \text { in } B_{1}(0), \\
g & =0, & \vec{G} & =\overrightarrow{0} & & \text { on } \partial B_{1}(0) .
\end{aligned}\right.
$$

Since $|\nabla \vec{\Phi}(x)| \simeq|x|^{\theta_{0}-1}$ near the origin and $\vec{F}$ is the fundamental solution of the 
Laplacian, by applying Calderón-Zygmund estimates to (2-13), we find ${ }^{13}$

$$
\nabla^{2} g, \nabla^{2} \vec{G} \in \begin{cases}L^{2, \infty}\left(B_{1}(0)\right), & \theta_{0}=1, \\ \operatorname{BMO}\left(B_{1}(0)\right), & \theta_{0} \geq 2 .\end{cases}
$$

In [Bernard and Rivière 2011b] (see Lemma A.2), the authors derive the identi$\operatorname{ties}^{14}$

$$
\left\{\begin{aligned}
\nabla \vec{\Phi} \cdot\left(\nabla^{\perp} \vec{L}+\nabla \vec{F}\right) & =0, \\
\nabla \vec{\Phi} \wedge\left(\nabla^{\perp} \vec{L}+\nabla \vec{F}\right) & =-2 \nabla \vec{\Phi} \wedge \nabla \vec{H}
\end{aligned}\right.
$$

Accounted into (2-13), the latter yield that we have, in $\Omega$,

$$
\left\{\begin{array}{r}
\operatorname{div}\left(\vec{L} \cdot \nabla^{\perp} \vec{\Phi}-\nabla g\right)=0, \\
\operatorname{div}\left(\vec{L} \wedge \nabla^{\perp} \vec{\Phi}-2 \vec{H} \wedge \nabla \vec{\Phi}-\nabla \vec{G}\right)=\overrightarrow{0},
\end{array}\right.
$$

where we have used the fact that

$$
\Delta \vec{\Phi} \wedge \vec{H}=2 \mathrm{e}^{2 \lambda} \vec{H} \wedge \vec{H}=\overrightarrow{0} .
$$

The terms under the divergence symbols in (2-16) both belong to $L^{2}\left(B_{1}(0)\right)$, owing to (2-12) and (2-14). The distributional equations (2-16), which are $a$ priori to be understood on $\Omega$, may thus be extended to all of $B_{1}(0)$. Indeed, a classical result of Laurent Schwartz states that the only distributions supported on $\{0\}$ are linear combinations of derivatives of the Dirac delta mass. Yet, none of these (including delta itself) belongs to $W^{-1,2}$. We shall thus understand (2-16) on $B_{1}(0)$. It is not difficult to verify (see Corollary IX.5 in [Dautray and Lions 1984]) that a divergence-free vector field in $L^{2}\left(B_{1}(0)\right)$ is the curl of an element in $W^{1,2}\left(B_{1}(0)\right)$. We apply this observation to (2-16) so as to infer the existence of two functions ${ }^{15}$ $S$ and $\vec{R}$ in the space $W^{1,2}\left(B_{1}(0)\right) \cap C^{\infty}(\Omega)$, with

$$
\left\{\begin{array}{l}
\nabla^{\perp} S=\vec{L} \cdot \nabla^{\perp} \vec{\Phi}-\nabla g \\
\nabla^{\perp} \vec{R}=\vec{L} \wedge \nabla^{\perp} \vec{\Phi}-2 \vec{H} \wedge \nabla \vec{\Phi}-\nabla \vec{G}
\end{array}\right.
$$

According to the identities (B-14) in the appendix, the functions $S$ and $\vec{R}$ satisfy on $B_{1}(0)$ the following system of equations, called the conservative conformal

${ }^{13}$ The weak- $L^{2}$ Marcinkiewicz space $L^{2, \infty}\left(B_{1}(0)\right)$ is defined as those functions $f$ which satisfy $\sup _{\alpha>0} \alpha^{2}\left|\left\{x \in B_{1}(0):|f(x)| \geq \alpha\right\}\right|<\infty$. In dimension two, the prototype element of $L^{2, \infty}$ is $|x|^{-1}$. The space $L^{2, \infty}$ is also a Lorentz space, and in particular is a space of interpolation between Lebesgue spaces, which justifies the first inclusion in (2-14). See [Hélein 1996] or [Almeida 1995] for details.

${ }^{14}$ Observe that $\nabla^{\perp} \vec{L}+\nabla \vec{F}$ is exactly the divergence-free quantity appearing in (1-7).

${ }^{15} S$ is a scalar while $\vec{R}$ is $\bigwedge^{2}\left(\mathbb{R}^{m}\right)$-valued. 
Willmore system: ${ }^{16}$

$$
\left\{\begin{array}{l}
-\Delta S=\nabla(\star \vec{n}) \cdot \nabla^{\perp} \vec{R}+\operatorname{div}((\star \vec{n}) \cdot \nabla \vec{G}), \\
-\Delta \vec{R}=\nabla(\star \vec{n}) \cdot \nabla^{\perp} \vec{R}-\nabla(\star \vec{n}) \cdot \nabla^{\perp} S+\operatorname{div}((\star \vec{n}) \cdot \nabla \vec{G}+\star \vec{n} \nabla g) .
\end{array}\right.
$$

Not only is this system independent of the codimension (which enters the equations in the guise of the operators $\star$ and $\bullet$ ), but it further displays two fundamental advantages. Analytically, (2-18) is uniformly elliptic. This is in sharp contrast with the Willmore equation (1-6) whose leading order operator $\Delta_{\perp}$ degenerates at the origin, owing to the presence of the conformal factor $\mathrm{e}^{\lambda(x)} \simeq|x|^{\theta_{0}-1}$. Structurally, the system (2-18) is in divergence form. We shall in the sequel capitalize on this remarkable feature to develop arguments of "integration by compensation". $A$ priori however, since $\vec{n}, S$, and $\vec{R}$ are elements of $W^{1,2}$, the leading terms on the right-hand side of the conservative conformal Willmore system (2-18) are critical. This difficulty is nevertheless bypassed using the fact that the $W^{1,2}$-norm of the Gauss map $\vec{n}$ is chosen to be small enough (see (1-4)).

2B. The general case when $\theta_{0} \geq 1$. We have gathered enough information about the functions involved to apply to the system (2-18) (a slightly extended version of) Proposition C.1 and thereby obtain that

$$
\nabla S, \nabla \vec{R} \in L^{p}\left(B_{1}(0)\right) \text { for some } p>2 .
$$

It is shown at the end of Section B in the appendix that

$$
2 \Delta \vec{\Phi}=\left(\nabla S-\nabla^{\perp} g\right) \cdot \nabla^{\perp} \vec{\Phi}-\left(\nabla \vec{R}-\nabla^{\perp} \vec{G}\right) \cdot \nabla^{\perp} \vec{\Phi} .
$$

Hence, as $|\nabla \vec{\Phi}(x)| \simeq \mathrm{e}^{\lambda(x)} \simeq|x|^{\theta_{0}-1}$ around the origin, using (2-14) and (2-19), we may call upon Proposition C.2 with the weight $|\mu|=\mathrm{e}^{\lambda}$ and $a=\theta_{0}-1$ to conclude that

$$
\left(\partial_{x_{1}}+i \partial_{x_{2}}\right) \vec{\Phi}(x)=\vec{P}(\bar{x})+\mathrm{e}^{\lambda(x)} \vec{T}(x)
$$

where $\vec{P}$ is a $\mathbb{C}^{m}$-valued polynomial of degree at most $\left(\theta_{0}-1\right)$, and $\vec{T}(x)=$ $\mathrm{O}\left(|x|^{1-2 / p-\epsilon}\right)$ for every $\epsilon>0$. Because $\mathrm{e}^{-\lambda} \nabla \vec{\Phi}$ is a bounded function, we deduce more precisely that $\vec{P}(\bar{x})=\theta_{0} \vec{A}^{*} \bar{x}^{\theta_{0}-1}$, for some constant vector $\vec{A} \in \mathbb{C}^{m}$ (we denote its complex conjugate by $\vec{A}^{*}$ ), so that

$$
\nabla \vec{\Phi}(x)=\left(\begin{array}{c}
\mathfrak{R} \\
-\Im
\end{array}\right)\left(\theta_{0} \vec{A} x^{\theta_{0}-1}\right)+\mathrm{e}^{\lambda(x)} \vec{T}(x) .
$$

Equivalently, upon writing $\vec{A}=\vec{A}^{1}+i \vec{A}^{2}$, where $\vec{A}^{1}$ and $\vec{A}^{2}$ are two vectors in $\mathbb{R}^{m}$,

\footnotetext{
${ }^{16}$ Refer to Appendix A for the notation and the operators used.
} 
the latter may be recast as

$$
\left\{\begin{aligned}
\partial_{x_{1}} \vec{\Phi}(x) & =\theta_{0}|x|^{\theta_{0}-1}\left[\vec{A}^{1} \cos \left(\left(\theta_{0}-1\right) \varphi\right)-\vec{A}^{2} \sin \left(\left(\theta_{0}-1\right) \varphi\right)\right]+\mathrm{e}^{\lambda} \mathfrak{R}(\vec{T}(x)), \\
-\partial_{x_{2}} \vec{\Phi}(x) & =\theta_{0}|x|^{\theta_{0}-1}\left[\vec{A}^{2} \cos \left(\left(\theta_{0}-1\right) \varphi\right)+\vec{A}^{1} \sin \left(\left(\theta_{0}-1\right) \varphi\right)\right]-\mathrm{e}^{\lambda} \Im(\vec{T}(x)),
\end{aligned}\right.
$$

where $\varphi \in[0,2 \pi)$ denotes the argument of $x \in B_{1}(0)$. The conformality condition on $\vec{\Phi}$ shows easily that

$$
\left|\vec{A}^{1}\right|=\left|\vec{A}^{2}\right| \quad \text { and } \quad \vec{A}^{1} \cdot \vec{A}^{2}=0 .
$$

Yet more precisely, as $|\nabla \vec{\Phi}|^{2}=2 \mathrm{e}^{2 \lambda}$, we see that

$$
\left.\left|\vec{A}^{1}\right|=\left|\vec{A}^{2}\right|=\frac{1}{\theta_{0}} \lim _{x \rightarrow 0} \frac{\mathrm{e}^{\lambda(x)}}{|x|^{\theta_{0}-1}} \in\right] 0, \infty[.
$$

Because $\vec{\Phi}(0)=\overrightarrow{0}$, we obtain from (2-21) the local expansion

$$
\vec{\Phi}(x)=\Re\left(\vec{A} x^{\theta_{0}}\right)+\mathrm{O}\left(|x|^{\theta_{0}+1-\frac{2}{p}-\epsilon}\right) .
$$

Since $\pi_{\vec{n}} \nabla \vec{\Phi} \equiv \overrightarrow{0}$, we deduce from (2-21) that

$$
\pi_{\vec{n}(x)} \vec{A}=-\theta_{0}^{-1} x^{1-\theta_{0}} \mathrm{e}^{\lambda} \pi_{\vec{n}} \vec{T}^{*}(x)=\mathrm{O}\left(|x|^{1-\frac{2}{p}-\epsilon}\right) \text { for all } \epsilon>0 .
$$

Now let $\delta:=1-2 / p \in(0,1)$, and let $0<\eta<p$ be arbitrary. We choose some $\epsilon$ satisfying

$$
0<\epsilon<\frac{2 \eta}{p(p-\eta)} \equiv \delta-1+\frac{2}{p-\eta} .
$$

We have observed that $\pi_{\vec{n}} \vec{A}=\mathrm{O}\left(|x|^{\delta-\epsilon}\right)$; hence $\pi_{\vec{n}} \vec{A}=\mathrm{o}\left(|x|^{1-2 /(p-\eta)}\right)$, and, in particular, we find

$$
\frac{1}{|x|} \pi_{\vec{n}(x)} \vec{A} \in L^{p-\eta}\left(B_{1}(0)\right) \text { for all } \eta>0 .
$$

This fact shall be helpful in the sequel.

When $\theta_{0}=1$, one directly deduces from the standard Calderón-Zygmund theorem applied to (2-20) that $\nabla^{2} \vec{\Phi} \in L^{p}$. In that case, $\mathrm{e}^{\lambda}$ is bounded from above and below, and thus the identity

$$
|\nabla \vec{n}|=\mathrm{e}^{-\lambda}\left|\pi_{\vec{n}} \nabla^{2} \vec{\Phi}\right|
$$

(derived as (B-4) in the appendix) yields that $\nabla \vec{n} \in L^{p}$. When now $\theta_{0} \geq 2$, we must proceed slightly differently to obtain analogous results. From (1-10), we know that $|x| \nabla \lambda(x)$ is bounded across the unit disk. We may thus apply Proposition C.2(ii) 
to (2-20) with the weight $|\mu|=\mathrm{e}^{\lambda}$ and $a=\theta_{0}$. The required hypothesis (C-13) is fulfilled, and so we obtain

$$
\nabla^{2} \vec{\Phi}(x)=\theta_{0}\left(1-\theta_{0}\right)\left(\begin{array}{rr}
-\Re & \mathfrak{I} \\
\mathfrak{I} & \Re
\end{array}\right)\left(\vec{A} x^{\theta_{0}-2}\right)+\mathrm{e}^{\lambda(x)} \vec{Q}(x),
$$

where $\vec{A}$ is as in (2-21), and $\vec{Q}$ lies in $\mathbb{R}^{4} \otimes L^{p-\epsilon}\left(B_{1}(0), \mathbb{R}^{m}\right)$ for every $\epsilon>0$. The exponent $p>2$ is the same as in (2-19).

Since $\mathrm{e}^{\lambda(x)} \simeq|x|^{\theta_{0}-1}$, we obtain from (2-27) that

$$
\mathrm{e}^{-\lambda}\left|\pi_{\vec{n}} \nabla^{2} \vec{\Phi}\right| \lesssim|x|^{-1}\left|\pi_{\vec{n}} \vec{A}\right|+\left|\pi_{\vec{n}} \vec{Q}\right| .
$$

According to (2-25), the first summand on the right-hand side of the latter belongs to $L^{p-\eta}$ for all $\eta>0$. Moreover, we have seen that $\pi_{\vec{n}} \vec{Q}$ lies in $L^{p-\epsilon}$ for all $\epsilon>0$, whence it follows that $\mathrm{e}^{-\lambda} \pi_{\vec{n}} \nabla^{2} \vec{\Phi}$ is itself an element of $L^{p-\epsilon}$ for all $\epsilon>0$. Brought into (2-26), this information implies that

$$
\nabla \vec{n} \in L^{p-\epsilon}\left(B_{1}(0)\right) \text { for all } \epsilon>0 .
$$

In light of this new fact, we may now return to (2-18). In particular, recalling (2-14), we find

$$
\Delta S \equiv-\nabla(\star \vec{n}) \cdot\left(\nabla^{\perp} \vec{R}+\nabla \vec{G}\right)-(\star \vec{n}) \cdot \Delta \vec{G} \in L^{q}\left(B_{1}(0)\right),
$$

with

$$
\frac{1}{q}=\frac{1}{p}+\frac{1}{p-\epsilon} .
$$

We attract the reader's attention on an important phenomenon occurring when $\theta_{0}=1$. In this case, if the aforementioned value of $q$ exceeds 2 (i.e., if $p>4$ ), then $\Delta S \notin L^{q}$, but rather only $\Delta S \in L^{2, \infty}$. This integrability "barrier" stems from that of $\Delta \vec{G}$, as given in (2-14). The same considerations apply of course with $\vec{R}$ and $g$ in place of $S$ and $\vec{G}$, respectively.

Our findings so far may be summarized as follows:

$$
\nabla S, \nabla \vec{R} \in \begin{cases}W^{1,(2, \infty)} & \text { if } \theta_{0}=1 \text { and } p>4, \\ W^{1, q} & \text { otherwise. }\end{cases}
$$

With the help of the Sobolev embedding theorem (and a result of [Tartar 2007] stating that $\left.W^{1,(2, \infty)} \subset \mathrm{BMO}\right)$, we infer that

$$
\nabla S, \nabla \vec{R} \in \begin{cases}\mathrm{BMO} & \text { if } \theta_{0}=1 \text { and } p>4, \\ L^{\infty} & \text { if } \theta_{0} \geq 2 \text { and } p>4, \\ L^{s} & \text { if } \theta_{0} \geq 1 \text { and } p \leq 4,\end{cases}
$$

with

$$
\frac{1}{s}=\frac{1}{q}-\frac{1}{2}=\frac{1}{p}+\frac{1}{p-\epsilon}-\frac{1}{2}<\frac{1}{p} .
$$


Comparing (2-30) to (2-19), we see that the integrability has been improved. The process may thus be repeated until reaching that

$$
\nabla S, \nabla \vec{R} \in L^{b}\left(B_{1}(0)\right) \text { for all } b<\infty
$$

holds in all configurations. With the help of this newly found fact, we reapply Proposition C.2 so as to improve (2-29) and (2-28) to

$$
\nabla S, \nabla \vec{R} \in \begin{cases}W^{1,(2, \infty)}\left(B_{1}(0)\right) & \text { if } \theta_{0}=1, \\ W^{1, b}\left(B_{1}(0)\right) & \text { if } \theta_{0} \geq 2 \text { for all } b<\infty\end{cases}
$$

and

$$
\nabla \vec{n} \in L^{b}\left(B_{1}(0)\right) \text { for all } b<\infty .
$$

The $\varepsilon$-regularity in the form (2-4) then yields a pointwise estimate for the Gauss map. Namely, in a neighborhood of the origin,

$$
|\nabla \vec{n}(x)| \lesssim|x|^{-\epsilon} \quad \text { for all } \epsilon>0 .
$$

2C. The case $\boldsymbol{\theta}_{\mathbf{0}}=1$. We shall now investigate further the case $\theta_{0}=1$, when $|\nabla \vec{\Phi}| \simeq \mathrm{e}^{\lambda}$ is bounded from both above and below around the origin. Setting

$$
\vec{F}_{1}:=\nabla^{\perp} \vec{R}+\nabla \vec{G} \quad \text { and } \quad F_{2}:=\nabla^{\perp} S+\nabla g
$$

in (2-20) gives

$$
-2 \Delta \vec{\Phi}=F_{2} \cdot \nabla \vec{\Phi}-\vec{F}_{1} \cdot \nabla \vec{\Phi}
$$

According to (2-14) and (2-29), the right-hand side of the latter has bounded mean oscillations. Hence $\nabla^{2} \vec{\Phi} \in \bigcap_{p<\infty} L^{p}$. Using that $2 \mathrm{e}^{2 \lambda} \vec{H}=\Delta \vec{\Phi}$, we differentiate
(2-35) to obtain

$$
-4 \nabla\left(\mathrm{e}^{2 \lambda} \vec{H}\right)=\nabla F_{2} \cdot \nabla \vec{\Phi}-\nabla \vec{F}_{1} \cdot \nabla \vec{\Phi}+F_{2} \cdot \nabla^{2} \vec{\Phi}-\vec{F}_{1} \bullet \nabla^{2} \vec{\Phi},
$$

which, still owing to (2-14) and (2-29), and to the boundedness of $\nabla \vec{\Phi}$, shows that $\mathrm{e}^{2 \lambda} \vec{H} \in W^{1,(2, \infty)}$. As $\mathrm{e}^{ \pm \lambda}$ are bounded from below, we see that $\vec{H} \in$ BMO. Using that $\nabla \lambda \in L^{2}$ (see (1-10)), it follows that

$$
\nabla \vec{H}=\mathrm{e}^{-2 \lambda} \nabla\left(\mathrm{e}^{2 \lambda} \vec{H}\right)-2(\nabla \lambda) \vec{H} \in \bigcap_{p<2} L^{p}
$$

We shall now derive an asymptotic expansion for $\vec{H}(x)$ near the origin. To this end, we use a "generic" procedure, whose assumptions are fulfilled owing to our work from the previous section (in particular (2-32)) and to (2-36). 
Proposition 2.2. Let the immersion $\vec{\Phi}$ satisfy an expansion of the type (2-21) for all $p<\infty$. Suppose that $\vec{n} \in \bigcap_{p<\infty} W^{1, p}\left(B_{1}(0)\right)$ and $\vec{H} \in \bigcap_{p<2} W^{1, p}\left(B_{1}(0)\right)$. Then, locally around the origin,

$$
\vec{H}(x)+\vec{\gamma}_{0} \log |x| \in \bigcap_{p<\infty} W^{1, p}\left(B_{1}(0)\right),
$$

where $\vec{\gamma}_{0}$ is the residue defined in (2-7).

Proof. In order to derive this result, one must return to (1-7):

$$
\mathscr{L}(\vec{H}):=\operatorname{div}\left(\nabla \vec{H}-3 \pi_{\vec{n}} \nabla \vec{H}+\star\left(\nabla^{\perp} \vec{n} \wedge \vec{H}\right)\right)=0 \quad \text { on } B_{1}(0) \backslash\{0\} .
$$

Owing to the hypotheses on $\vec{n}$ and $\vec{H}$, this equation has a distributional sense. Since $\mathscr{L}(\vec{H})$ is supported on the origin and belongs to $W^{-1, p}$ for $p<2$, it can only be proportional to the Dirac mass $\delta_{0}$. From (2-7), we deduce more precisely that

$$
\mathscr{L}(\vec{H})=4 \pi \vec{\gamma}_{0} \delta_{0} .
$$

Let $\vec{A} \in \mathbb{C}^{m}$ be the constant vector appearing in the expansion (2-21). Since $\pi_{\vec{n}(0)} \vec{A}=\overrightarrow{0}$ (see (2-24)), an elementary computation gives

$$
\begin{aligned}
4 \pi \vec{A} \cdot \vec{\gamma}_{0} \delta_{0} & =4 \pi \pi_{T} \vec{A} \cdot \vec{\gamma}_{0} \delta_{0}=\pi_{T} \vec{A} \cdot \mathscr{L}(\vec{H}) \\
& =\operatorname{div}\left(\vec{H} \cdot \nabla \pi_{T} \vec{A}-\pi_{T} \vec{A} \cdot \star\left(\nabla^{\perp} \vec{n} \wedge \vec{H}\right)\right) \\
& \quad+\nabla \pi_{T} \vec{A} \cdot\left(\nabla \vec{H}-3 \pi_{\vec{n}} \nabla \vec{H}+\star\left(\nabla^{\perp} \vec{n} \wedge \vec{H}\right)\right),
\end{aligned}
$$

where we have used the fact that $\pi_{T} \vec{H} \equiv \overrightarrow{0}$.

Because $\vec{A}$ is constant and $\nabla \vec{n} \in \bigcap_{p<\infty} L^{p}$, it follows from the fact that $\pi_{\vec{n}}=$ $\vec{n}\left\llcorner\vec{n}\left\llcorner\right.\right.$ that $\nabla \pi_{\vec{n}} \vec{A}$ and thus $\nabla \pi_{T} \vec{A}$ lie in $\bigcap_{p<\infty} L^{p}$. Moreover, $\nabla \vec{H} \in \bigcap_{1 \leq p<2} L^{p}$ by hypothesis. Introducing this information into (2-37), we note that its right-hand side belongs to $W^{-1, p}$ for all $p<\infty$. Yet, its left-hand side is proportional to the Dirac mass, which does not belong to any $W^{-1, p}$ for $p \geq 2$. We accordingly conclude that $\vec{A} \cdot \vec{\gamma}_{0}=0$. Returning to the expansion (2-21) reveals now that

$$
\vec{\gamma}_{0} \cdot\left(\begin{array}{l}
\vec{e}_{1}(x) \\
\vec{e}_{2}(x)
\end{array}\right) \simeq \vec{\gamma}_{0} \cdot \vec{T}(x)=\mathrm{O}\left(|x|^{1-\epsilon}\right) \text { for all } \epsilon>0,
$$

whence

$$
|x|^{-1} \pi_{T}\left(\vec{\gamma}_{0}\right) \in \bigcap_{p<\infty} L^{p}\left(B_{1}(0)\right) .
$$

A direct computation gives

$$
\begin{aligned}
\mathscr{L}\left(\vec{\gamma}_{0} \log |x|\right) & =-4 \pi \vec{\gamma}_{0} \delta_{0}+\operatorname{div}\left(3 \pi_{T}\left(\vec{\gamma}_{0}\right) \nabla \log |x|+\star\left(\nabla^{\perp} \vec{n} \wedge \vec{\gamma}_{0}\right) \log |x|\right) \\
& =-\mathscr{L}(\vec{H})+\operatorname{div}\left(3 \pi_{T}\left(\vec{\gamma}_{0}\right) \nabla \log |x|+\star\left(\nabla^{\perp} \vec{n} \wedge \vec{\gamma}_{0}\right) \log |x|\right) .
\end{aligned}
$$


Using the fact that $\nabla \vec{n} \in \bigcap_{p<\infty} L^{p}$ and (2-38) shows that

$$
\mathscr{L}\left(\vec{H}+\vec{\gamma}_{0} \log |x|\right) \in \bigcap_{p<\infty} W^{-1, p} .
$$

It is established in [Rivière 2008] that the operator $\mathscr{L}$ is second-order elliptic and in particular that it satisfies $\mathscr{L}^{-1} W^{-1, p} \subset W^{1, p}$. The desired claim ensues:

$$
\vec{H}(x)+\vec{\gamma}_{0} \log |x| \in \bigcap_{p<\infty} W^{1, p} .
$$

We continue our study of the case $\theta_{0}=1$ by a slight improvement on the regularity of the Gauss map $\vec{n}$. It is shown as (B-7) in the appendix that the $\bigwedge^{m-2}\left(\mathbb{S}^{m-1}\right)$-valued Gauss map $\vec{n}$ satisfies a perturbed harmonic map equation, namely

$$
\Delta \vec{n}=2 \star\left(\nabla^{\perp} \vec{\Phi} \wedge \nabla \vec{H}\right)+2 \mathrm{e}^{2 \lambda} K \vec{n}-2 \star \mathrm{e}^{2 \lambda} \vec{h}_{12} \wedge\left(\vec{h}_{11}-\vec{h}_{22}\right),
$$

where $K$ denotes the Gauss curvature. Recall that

$$
|\nabla \vec{n}|=\mathrm{e}^{-\lambda}\left|\pi_{\vec{n}} \nabla^{2} \vec{\Phi}\right|=\mathrm{e}^{\lambda}\left|\begin{array}{ll}
\vec{h}_{11} & \vec{h}_{12} \\
\vec{h}_{21} & \vec{h}_{22}
\end{array}\right|,
$$

so that $\mathrm{e}^{\lambda} \vec{h}_{i j}$ inherits the regularity of $\nabla \vec{n} \in \bigcap_{p<\infty} L^{p}$. Bringing this information and the expansion given in Proposition 2.2 into (2-39) shows that

$$
|\Delta \vec{n}| \lesssim|x|^{-1}+\text { terms in } \bigcap_{p<\infty} L^{p} \in L^{2, \infty} .
$$

Hence $\nabla^{2} \vec{n} \in L^{2, \infty}$, and thus $\nabla \vec{n} \in \mathrm{BMO}$.

2D. The case $\boldsymbol{\theta}_{\mathbf{0}} \geq 2$. We now return to (2-20) in the case when $\theta_{0} \geq 2$. Setting

$$
\vec{F}_{1}:=\nabla^{\perp} \vec{R}+\nabla \vec{G} \quad \text { and } \quad F_{2}:=\nabla^{\perp} S+\nabla g,
$$

it reads

$$
-2 \Delta \vec{\Phi}=F_{2} \cdot \nabla \vec{\Phi}-\vec{F}_{1} \cdot \nabla \vec{\Phi} .
$$

Owing to (2-14) and (2-29), the functions $\vec{F}_{1}$ and $F_{2}$ are Hölder continuous of any order $\alpha \in(0,1)$. It thus makes sense to define the constants

$$
\vec{f}_{1}:=\vec{F}_{1}(0) \text { and } \quad f_{2}:=F_{2}(0) .
$$

They are elements of $\mathbb{R}^{2} \otimes \bigwedge^{2}\left(\mathbb{R}^{m}\right)$ and of $\mathbb{R}^{2}$, respectively. We will in the sequel view $\vec{f}_{1}$ as an element of $\mathbb{C} \otimes \bigwedge^{2}\left(\mathbb{R}^{m}\right)$ and $f_{2}$ as an element of $\mathbb{C}$.

For future purposes, let us define $\vec{\Gamma}$ via

$$
\Delta \vec{\Gamma}=4 \theta_{0} \Re\left(\vec{C}_{\theta_{0}-1}^{*} x^{\theta_{0}-1}\right) \quad \text { with }-8 \vec{C}_{\theta_{0}-1}^{*}:=f_{2} \vec{A}-\vec{f}_{1} \bullet \vec{A},
$$


where $\vec{A} \in \mathbb{C}^{m}$ is the constant vector appearing in (2-21). More precisely, writing $\vec{f}_{1}=\vec{f}_{1}^{1}+i \vec{f}_{1}^{2}$ and $f_{2}=f_{2}^{1}+i f_{2}^{2}$, then

$$
\left\{\begin{array}{l}
-8 \Re\left(\vec{C}_{\theta_{0}-1}^{*}\right)=f_{2}^{1} \vec{A}^{1}-f_{2}^{2} \vec{A}^{2}-\vec{f}_{1}^{1} \bullet \vec{A}^{1}+\vec{f}_{1}^{2} \cdot \vec{A}^{2}, \\
-8 \Im\left(\vec{C}_{\theta_{0}-1}^{*}\right)=f_{2}^{1} \vec{A}^{2}+f_{2}^{2} \vec{A}^{1}-\vec{f}_{1}^{1} \cdot \vec{A}^{2}-\vec{f}_{1}^{2} \cdot \vec{A}^{1} .
\end{array}\right.
$$

Equation (2-42) is solved explicitly (up to an unimportant harmonic function):

$$
\vec{\Gamma}(x)=\Re\left(\vec{C}_{\theta_{0}-1}|x|^{2 \theta_{0}} x^{1-\theta_{0}}\right),
$$

where $\vec{C}_{\theta_{0}-1}$ is the complex conjugate of $\vec{C}_{\theta_{0}-1}^{*}$.

Note next that (2-41) and (2-42) yield

$$
2 \Delta(\vec{\Phi}-\vec{\Gamma})=\left(F_{2}-f_{2}\right) \cdot \nabla \vec{\Phi}-\left(\vec{F}_{1}-\vec{f}_{1}\right) \cdot \nabla \vec{\Phi}+\mathrm{e}^{\lambda}\left[f_{2} \cdot \vec{T}-\vec{f}_{1} \cdot \vec{T}\right]
$$

where we have used the representation (2-21).

Since $|\vec{T}(x)| \lesssim|x|^{1-\epsilon}$ for all $\epsilon>0$, and $\left|\vec{F}_{1}(x)-\vec{f}_{1}\right|+\left|F_{2}(x)-f_{2}\right| \lesssim|x|^{\alpha}$ for all $\alpha \in(0,1)$, while, as previously explained, the weight $|\nabla \vec{\Phi}(x)| \simeq \mathrm{e}^{\lambda}$ satisfies the condition (C-13), we may apply Corollary C.3 to (2-44), thereby obtaining

$$
\begin{aligned}
\nabla(\vec{\Phi}-\vec{\Gamma})(x) & =\vec{P}(\bar{x})+\mathrm{e}^{\lambda(x)} \vec{U}(x), \\
\nabla^{2}(\vec{\Phi}-\vec{\Gamma})(x) & =\nabla \vec{P}(\bar{x})+\mathrm{e}^{\lambda(x)} \vec{V}(x),
\end{aligned}
$$

where $\vec{P}$ is a polynomial of degree at most $\theta_{0}$. Moreover, $\vec{U}(x)=\mathrm{O}\left(|x|^{2-\epsilon}\right)$, and

$$
|x|^{-(1-\epsilon)} \vec{V} \in \bigcap_{p<\infty} L^{p} \quad \text { with } \operatorname{Tr} \vec{V}(x)=\mathrm{O}\left(|x|^{1-\epsilon}\right) \quad \text { for all } \epsilon>0 .
$$

One sees in (2-43) that $\nabla \vec{\Gamma}(x)=\mathrm{O}\left(|x|^{\theta_{0}}\right)$. Hence, from (2-45) and the fact that $|\nabla \vec{\Phi}|(x) \simeq|x|^{\theta_{0}-1}$, it follows that the polynomial $\vec{P}$ contains exactly one monomial of degree $\left(\theta_{0}-1\right)$ and one monomial of degree $\theta_{0}$. More precisely, identifying the representations (2-27) and (2-46) yields

$$
\begin{aligned}
\nabla^{2} \vec{\Phi}(x)=\left(\begin{array}{rr}
-\Re & \Im \\
\Im & \Re
\end{array}\right)\left(\theta_{0}\left(1-\theta_{0}\right) \vec{A} x^{\theta_{0}-2}-\theta_{0}\left(1+\theta_{0}\right) \vec{B}_{1} x^{\theta_{0}-1}\right) \\
+\nabla^{2} \vec{\Gamma}(x)+\mathrm{e}^{\lambda(x)} \vec{V}(x),
\end{aligned}
$$

where $\vec{B}_{1} \in \mathbb{C}^{m}$ is constant. The constant vector $\vec{A}$ is as in (2-21).

Remark 2.3. The estimates (2-47) may be slightly improved. To do so, one differentiates (2-44) throughout with respect to $x_{j}$, and applies Proposition C.2(i) and Corollary C.3 to the resulting equation (however, Proposition C.2(ii) will not be available, as some of the weights appearing — of the order $\left|\nabla^{2} \vec{\Phi}\right|$ - need not 
a priori fulfill the condition (C-13)). One eventually obtains that

$$
\vec{V}(x)=\mathrm{O}\left(|x|^{1-\epsilon}\right) \text { for all } \epsilon>0 .
$$

Now recall that $|\nabla \vec{\Phi}(0)|=0=|\vec{\Phi}(0)|$. We deduce from (2-48) and (2-43) that

$$
\vec{\Phi}(x)=\Re\left(\vec{A} x^{\theta_{0}}+\vec{B}_{1} x^{\theta_{0}+1}+\vec{C}_{\theta_{0}-1}|x|^{2 \theta_{0}} x^{1-\theta_{0}}\right)+|x|^{\theta_{0}-1} \vec{\xi}(x),
$$

where

$\vec{\xi}(x)=\mathrm{O}\left(|x|^{3-\epsilon}\right), \quad \nabla \vec{\xi}(x)=\mathrm{O}\left(|x|^{2-\epsilon}\right), \quad \nabla^{2} \vec{\xi}(x)=\mathrm{O}\left(|x|^{1-\epsilon}\right) \quad$ for all $\epsilon>0$.

Moreover, as $2 \mathrm{e}^{2 \lambda} \vec{H}=\Delta \vec{\Phi}$, the representation (2-48) along with (2-42) gives the local asymptotic expansion

$$
\vec{H}(x)=2 \theta_{0} \mathrm{e}^{2 u(x)} \Re\left(\vec{C}_{\theta_{0}-1} x^{1-\theta_{0}}\right)+\mathrm{O}\left(|x|^{2-\theta_{0}-\epsilon}\right) \text { for all } \epsilon>0,
$$

where $\vec{C}_{\theta_{0}-1}$ is as above, and $\mathrm{e}^{u(x)}:=|x|^{1-\theta_{0}} \mathrm{e}^{\lambda(x)}$, which is known to have a positive limit at the origin. This shows in particular that $\mathrm{e}^{\lambda(x)} \vec{H}(x)$ is a bounded function (unlike the case $\theta_{0}=1$, whereby $\mathrm{e}^{\lambda} \vec{H} \simeq \vec{H}$ behaves logarithmically). However, it "spins" as $x$ approaches the origin: its limit need not exist, and, if it does exist, then it must be zero (i.e., $\vec{C}_{\theta_{0}-1}=\overrightarrow{0}$ ). Note in addition that, because $\vec{H}$ is a normal vector, we have always $\pi_{\vec{n}(0)} \vec{C}_{\theta_{0}-1}=\vec{C}_{\theta_{0}-1}$.

We close this section by proving that $\nabla^{2} \vec{n} \in L^{2, \infty}$ and that $\nabla \vec{n} \in L^{\infty}$. We have seen that $\mathrm{e}^{\lambda} \vec{H}$ is bounded. Applying standard elliptic techniques to (1-7) then yields that $|x| \mathrm{e}^{\lambda} \nabla \vec{H}$ is bounded as well, and hence that $\mathrm{e}^{\lambda} \nabla \vec{H} \in L^{2, \infty}$. Going back to the perturbed harmonic map equation (2-39) satisfied by the Gauss map $\vec{n}$, and using the fact that $\mathrm{e}^{\lambda} \vec{h}_{i j}$ inherits the regularity of $\nabla \vec{n} \in \bigcap_{p<\infty} L^{p}$, we deduce that $\Delta \vec{n}$ lies in $L^{2, \infty}$, and therefore indeed that $\nabla^{2} \vec{n} \in L^{2, \infty}$. In particular, this implies that $\nabla \vec{n} \in \mathrm{BMO}$. However, it is possible to show that $\nabla \vec{n} \in L^{\infty}\left(B_{1}(0)\right)$. To see this, we first note that (2-50) yields

$$
\nabla \vec{\Phi}(x)=\left(\begin{array}{c}
\mathfrak{R} \\
-\mathfrak{\Im}
\end{array}\right)\left(\theta_{0} \vec{A} x^{\theta_{0}-1}\right)+\nabla\left(|x|^{\theta_{0}-1} \vec{\xi}(x)\right)+\mathrm{O}\left(|x|^{\theta_{0}}\right) .
$$

Since $\pi_{\vec{n}} \nabla \vec{\Phi} \equiv 0$, the latter and the estimates on $\vec{\xi}$ give

$$
\left|\pi_{\vec{n}(x)} \vec{A}\right|=\mathrm{O}(|x|) .
$$

A quick inspection of the identity (2-48) then reveals that

$$
\left|\pi_{\vec{n}(x)} \nabla^{2} \vec{\Phi}(x)\right| \lesssim\left|\pi_{\vec{n}(x)} \vec{A}\right||x|^{\theta_{0}-2}+\mathrm{O}\left(|x|^{\theta_{0}-1}\right)=\mathrm{O}\left(|x|^{\theta_{0}-1}\right) .
$$

Combining this with (2-26) gives that $\nabla \vec{n}$ is bounded across the singularity. 
2D1. An observation. In this section, we adopt the previously encountered complex notation $x:=x_{1}+i x_{2}$ and $\bar{x}:=x_{1}-i x_{2}$. We set $\partial_{x}:=\frac{1}{2}\left(\partial_{x_{1}}-i \partial_{x_{2}}\right)$ and $\partial_{\bar{x}}:=\frac{1}{2}\left(\partial_{x_{1}}+i \partial_{x_{2}}\right)$. We may then deduce from (2-50) that

$$
2 \partial_{x} \vec{\Phi}=\theta_{0} \vec{A} x^{\theta_{0}-1}+\mathrm{O}\left(|x|^{\theta_{0}}\right),
$$

and thus

$$
\vec{A}=\frac{2}{\theta_{0}} \lim _{x \rightarrow 0} x^{1-\theta_{0}} \partial_{x} \vec{\Phi} .
$$

On the other hand, when $\theta_{0} \geq 2$, recalling (2-40) and (2-17), we have

$$
\begin{aligned}
& \vec{F}_{1}:=\nabla^{\perp} \vec{R}+\nabla \vec{G}=\vec{L} \wedge \nabla^{\perp} \vec{\Phi}-2 \vec{H} \wedge \nabla \vec{\Phi} \equiv 2 i(\vec{L}+2 i \vec{H}) \wedge \partial_{\bar{x}} \vec{\Phi}, \\
& F_{2}:=\nabla^{\perp} S+\nabla g=\vec{L} \cdot \nabla^{\perp} \vec{\Phi} \equiv 2 i \vec{L} \cdot \partial_{\bar{x}} \vec{\Phi}
\end{aligned}
$$

From (2-42), we now find

$$
\begin{aligned}
-8 \vec{C}_{\theta_{0}-1}^{*} & :=F_{2}(0) \vec{A}-\vec{F}_{1}(0) \cdot \vec{A} \\
& =\frac{4 i}{\theta_{0}} \lim _{x \rightarrow 0} x^{1-\theta_{0}}\left[\vec{L} \cdot \partial_{\bar{x}} \vec{\Phi}\right] \partial_{x} \vec{\Phi}-\frac{4 i}{\theta_{0}} \lim _{x \rightarrow 0} x^{1-\theta_{0}}\left[(\vec{L}+2 i \vec{H}) \wedge \partial_{\bar{x}} \vec{\Phi}\right] \bullet \partial_{x} \vec{\Phi} .
\end{aligned}
$$

Rearranging the computations leading to the identities (B-11) yields without much effort that

$$
\left(\vec{V} \wedge \partial_{\bar{x}} \vec{\Phi}\right) \cdot \partial_{x} \vec{\Phi}=\left(\vec{V} \cdot \partial_{\bar{x}} \vec{\Phi}\right) \partial_{x} \vec{\Phi}+\frac{1}{2} \mathrm{e}^{2 \lambda} \vec{V}
$$

holds for all 1 -vectors $\vec{V}$. As $\vec{H}$ is a normal vector, we thus find

$$
-8 \vec{C}_{\theta_{0}-1}^{*}=-\frac{2 i}{\theta_{0}} \lim _{x \rightarrow 0} x^{1-\theta_{0}} \mathrm{e}^{2 \lambda}(\vec{L}+2 i \vec{H}) .
$$

Introducing, as in (2-51), the function $\mathrm{e}^{u(x)}:=|x|^{1-\theta_{0}} \mathrm{e}^{\lambda(x)}$, which is known to be continuous, bounded from above and below across the origin, we reach the expression

$$
-\vec{C}_{\theta_{0}-1}=\frac{\mathrm{e}^{2 u(0)}}{4 \theta_{0}} \lim _{x \rightarrow 0} x^{\theta_{0}-1}(2 \vec{H}+i \vec{L})
$$

The importance of the function $2 \vec{H}+i \vec{L}$ further arises in Section 2E.

\section{E. Removability results.}

2E1. Preparation. We now return to the defining equation for $\vec{L}$, namely

$$
\nabla^{\perp} \vec{L}:=\nabla \vec{H}-3 \pi_{\vec{n}} \nabla \vec{H}+\star\left(\nabla^{\perp} \vec{n} \wedge \vec{H}\right)-2 \vec{\gamma}_{0} \nabla \log |x| .
$$


We will first recast this equation in the form ${ }^{17}$

$$
\nabla^{\perp} \vec{L}=-2 \nabla \vec{H}+3 \pi_{T} \nabla \vec{H}-\star\left(\vec{n} \wedge \pi_{T} \nabla^{\perp} \vec{H}\right)-2 \vec{\gamma}_{0} \nabla \log |x|,
$$

where we have used the fact that $\vec{H}$ is a normal vector, so $\vec{n} \wedge \vec{H}=\overrightarrow{0}$. In turn, the latter is equivalently expressed as ${ }^{18}$

$$
\partial_{x}\left(\vec{L}+2 i \vec{H}+2 i \vec{\gamma}_{0} \log |x|\right)=3 i \pi_{T} \partial_{x} \vec{H}-\star\left(\vec{n} \wedge \pi_{T} \partial_{x} \vec{H}\right) .
$$

Using the fact that $\vec{H}$ is normal and (B-2), a simple computation reveals that

$$
\pi_{T} \partial_{x} \vec{H}=-\sum_{j=1,2}\left(\vec{H} \cdot \pi_{\vec{n}} \partial_{x} \vec{e}_{j}\right) \vec{e}_{j}=-(\vec{H} \cdot \vec{H}) \partial_{x} \vec{\Phi}-\left(\vec{H} \cdot \overline{\vec{H}}_{0}\right) \partial_{\bar{x}} \vec{\Phi},
$$

where $\vec{H}_{0}$ denotes the Weingarten operator:

$$
\vec{H}_{0}:=\frac{1}{2}\left(\vec{h}_{11}-\vec{h}_{22}-2 i \vec{h}_{12}\right) .
$$

From this and the elementary identities

$$
\star\left(\vec{n} \wedge \partial_{x} \vec{\Phi}\right)=i \partial_{x} \vec{\Phi} \quad \text { and } \quad \star\left(\vec{n} \wedge \partial_{\bar{x}} \vec{\Phi}\right)=-i \partial_{\bar{x}} \vec{\Phi}
$$

we obtain

$$
\star\left(\vec{n} \wedge \pi_{T} \partial_{x} \vec{H}\right)=-i(\vec{H} \cdot \vec{H}) \partial_{x} \vec{\Phi}+i\left(\vec{H} \cdot \vec{H}_{0}\right) \partial_{\bar{x}} \vec{\Phi} .
$$

Altogether (2-59)-(2-60) brought into (2-58) give

$$
\partial_{x}\left(i \vec{L}-2 \vec{H}-2 \vec{\gamma}_{0} \log |x|\right)=2(\vec{H} \cdot \vec{H}) \partial_{x} \vec{\Phi}+4\left(\vec{H} \cdot \vec{H}_{0}\right) \partial_{\bar{x}} \vec{\Phi}
$$

This equation, like the original one introducing $\vec{L}$, is valid only on the punctured disk $D^{2} \backslash\{0\}$. For notational convenience, we will henceforth write it

$$
\partial_{x}\left(i \vec{L}-2 \vec{H}-2 \vec{\gamma}_{0} \log |x|\right)=2 \vec{q} \text {. }
$$

Owing to the fact that $|\vec{H}||\nabla \vec{\Phi}|$ and $\left|\vec{H}_{0}\right||\nabla \vec{\Phi}|$ are controlled by $|\nabla \vec{n}|$, we note that

$$
|\vec{q}| \lesssim|\nabla \vec{n}||\vec{H}| .
$$

Lemma 2.4. If, locally around the origin, for some integer $k \in\left\{1, \ldots, \theta_{0}\right\}$, we have

$$
\vec{H}=\mathrm{O}\left(|x|^{k-\theta_{0}-\epsilon}\right) \quad \text { for all } \epsilon>0,
$$

\footnotetext{
${ }^{17}$ Recall that $\pi_{T}:=\mathrm{id}-\pi_{\vec{n}}$ denotes projection onto the tangent space.
}

${ }^{18}$ With the same notation as in Section 2D1. 
then we have

$$
\vec{H}+\vec{\gamma}_{0} \log |x|-\frac{i}{2} \vec{L}=\vec{E}-\vec{T}
$$

The function $\vec{E}$ is antimeromorphic with a pole at the origin of order at most $\left(\theta_{0}-k\right)$. Moreover,

$$
\partial_{x} \vec{T}=\vec{q} \quad \text { on } D^{2} \backslash\{0\}, \quad \vec{T}=\mathrm{O}\left(|x|^{1+k-\theta_{0}-\epsilon}\right) \quad \text { for all } \epsilon>0
$$

The function $\vec{T}$ is unique up to addition of antimeromorphic summands.

Proof. Suppose that for some integer $k \in\left\{1, \ldots, \theta_{0}\right\}$ we have

$$
\vec{H}=\mathrm{O}\left(|x|^{k-\theta_{0}-\epsilon}\right) \quad \text { for all } \epsilon>0 \text {. }
$$

Owing to (2-63), we have as well

$$
|x|^{\theta_{0}-k} \vec{q} \in \bigcap_{p<\infty} L^{p}
$$

We consider any $\vec{w}$ satisfying

$$
\partial_{x} \vec{w}=2 \bar{x}^{\theta_{0}-k} \vec{q} \quad \text { on } D^{2}
$$

Per (2-65), $\vec{w}$ is $C^{0,1-\epsilon}$-Hölder continuous for any $\epsilon>0$. From (2-61), we have

$$
\partial_{x}\left[\bar{x}^{\theta_{0}-k}\left(i \vec{L}-2 \vec{H}-2 \vec{\gamma}_{0} \log |x|\right)-\vec{w}\right]=0 \quad \text { on } D^{2} \backslash\{0\} .
$$

We will extend this equation to all of the unit disk $D^{2}$. To do so, it suffices to show that the function to which the operator $\partial_{x}$ is applied on the left-hand side of (2-67) lies in $L^{2}$. Since $\vec{w}$ is Hölder continuous, while $\vec{H}$ satisfies (2-63), it only remains to verify that $|x|^{\theta_{0}-k} \vec{L}$ lies in the space $L^{2}$. Exactly as we derived (2-12) from (2-5), we infer here that $|x|^{\theta_{0}+1-k} \nabla \vec{H} \in \bigcap_{p<\infty} L^{p}$, and then per (2-57) that

$$
|x|^{\theta_{0}+1-k} \nabla \vec{L} \in \bigcap_{p<\infty} L^{p},
$$

from which we obtain that $|x|^{\theta_{0}-k} \vec{L} \in L^{2}$. Accordingly, (2-67) holds on the unit disk, whence

$$
\vec{H}+\vec{\gamma}_{0} \log |x|-\frac{i}{2} \vec{L}=\vec{P}-\bar{x}^{k-\theta_{0}} \vec{w}
$$

where $\vec{P}$ is antimeromorphic with a pole at the origin of order at most $\left(\theta_{0}-k\right)$. Putting in the latter

$$
\vec{E}:=\vec{P}+\bar{x}^{k-\theta_{0}} \vec{w}(0) \quad \text { and } \quad \vec{T}:=(\vec{w}-\vec{w}(0)) \bar{x}^{k-\theta_{0}}
$$


gives the desired representation (2-64). Moreover, we have

$$
\partial_{x} \vec{T}=\vec{q} \quad \text { on } D^{2} \backslash\{0\}, \quad \vec{T}=\mathrm{O}\left(|x|^{1+k-\theta_{0}-\epsilon}\right) \text { for all } \epsilon>0 .
$$

The function $\vec{w}$ is clearly unique up to addition of antimeromorphic terms. The same is also true for $\vec{T}$. Should the "first" found $\vec{T}$ happen to contain an antimeromorphic summand, it will necessarily be of order at most $\mathrm{O}\left(|x|^{1+k-\theta_{0}}\right)$ and could thus safely be fed into $\vec{E}$ without affecting the desired statement.

We now come to a central result in our study.

Proposition 2.5. There exists a unique function $\vec{T}$ containing no monomial of $\bar{x}$, satisfying

$$
\partial_{x} \vec{T}=\vec{q} \quad \text { on } D^{2} \backslash\{0\} \quad \text { and } \quad \vec{T}=\mathrm{O}\left(|x|^{2-\theta_{0}-\epsilon}\right) \quad \text { for all } \epsilon>0,
$$

and such that locally around the singularity, we have

$$
\vec{H}(x)+\vec{\gamma}_{0} \log |x|-\frac{i}{2} \vec{L}(x)=\vec{E}(x)-\vec{T}(x),
$$

where $\vec{\gamma}_{0}$ is the residue defined in (1-8), while the function $\vec{E}$ is antiholomorphic with possibly a pole at the origin of order at most $\left(\theta_{0}-1\right)$.

If the singularity of $\vec{E}$ at the origin has order $\alpha \in\left\{0, \ldots, \theta_{0}-1\right\}$, then $\vec{E}$ and $\vec{T}$ may be adjusted to satisfy

$$
\vec{E}-\vec{T}=\vec{E}_{\alpha} \bar{x}^{-\alpha}-\vec{Q}
$$

for some nonzero constant $\vec{E}_{\alpha} \in \mathbb{C}^{m}$, and with

$$
\partial_{x} \vec{Q}=\vec{q} \quad \text { on } D^{2} \backslash\{0\} \quad \text { and } \quad \vec{Q}=\mathrm{O}\left(|x|^{1-\alpha-\epsilon}\right) \text { for all } \epsilon>0 .
$$

Proof. We have seen in Proposition 2.2 and in (2-51) that $\vec{H}=\mathrm{O}\left(|x|^{1-\theta_{0}-\epsilon}\right)$ for all $\epsilon>0$. The desired representation (2-70) was obtained in Lemma 2.4.

For simplicity, we will only prove the second part of the lemma for the first three cases $\alpha \in\left\{\theta_{0}-3, \ldots, \theta_{0}-1\right\}$. All other cases are obtained mutatis mutandis.

Case $\alpha=\theta_{0}-1$. We can write locally

$$
\vec{E}=\vec{E}_{\theta_{0}-1} \bar{x}^{1-\theta_{0}}+\vec{E}^{0},
$$

where $\vec{E}_{\theta_{0}-1} \in \mathbb{C}^{m}$ is constant, and $\vec{E}^{0}$ is an antimeromorphic function with a pole at the origin of order at most $\left(\theta_{0}-2\right)$; i.e., $\left|\vec{E}^{0}\right| \lesssim|x|^{2-\theta_{0}}$. We may then let $\vec{Q}:=\vec{T}-\vec{E}^{0}$ with $\partial_{x} \vec{Q}=\partial_{x} \vec{T}$ on $D^{2} \backslash\{0\}$, and $\vec{Q}$ and $\vec{T}$ have the same asymptotic behavior at the origin. 
Case $\alpha=\theta_{0}-2$. In this case, without loss of generality, $\theta_{0} \geq 2$, so that $\nabla \vec{n} \in L^{\infty}$.

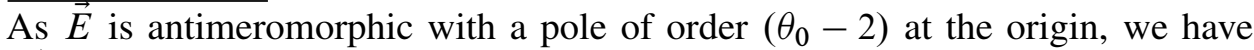
$|\vec{E}| \simeq|x|^{2-\theta_{0}}$ near the origin. The second condition in (2-69) put into (2-70) shows that $\vec{H}$ is controlled by $|x|^{2-\theta_{0}-\epsilon}$ for all $\epsilon>0$. Calling upon the representation (2-64) with $k=2$ gives that

$$
-\frac{i}{2} \vec{L}+\vec{H}+\vec{\gamma}_{0} \log |x|=\vec{E}^{1}-\vec{T}^{1},
$$

where $\vec{E}^{1}$ is an antimeromorphic function with a pole at the origin of order at most $\left(\theta_{0}-2\right)$, and a function $\vec{T}^{1}$ satisfies

$$
\partial_{x} \vec{T}^{1}=\vec{q} \quad \text { and } \quad \vec{T}^{1}=\mathrm{O}\left(|x|^{3-\theta_{0}-\epsilon}\right) \quad \text { for all } \epsilon>0 .
$$

As we did in the case $\alpha=\theta_{0}-1$, we can write

$$
\vec{E}^{1}(\bar{x})=\vec{E}_{\theta_{0}-2} \bar{x}^{2-\theta_{0}}+\vec{E}^{0}(\bar{x}),
$$

where $\vec{E}_{\theta_{0}-2} \in \mathbb{C}^{m}$ is constant, and $\vec{E}^{0}$ is an antimeromorphic function with a pole at the origin of order at most $\left(\theta_{0}-3\right)$. Clearly, the function $\vec{Q}:=\vec{T}^{1}-\vec{E}^{0}$ satisfies the two conditions (2-71). Furthermore, we have

$$
-\frac{i}{2} \vec{L}+\vec{H}+\vec{\gamma}_{0} \log |x|=\vec{E}^{1}-\vec{T}^{1}=\vec{E}_{\theta_{0}-2} \bar{x}^{2-\theta_{0}}-\vec{Q},
$$

as desired.

Case $\alpha=\theta_{0}-3$. We start with the representation (2-64) with $k=1$, which as we have seen is equivalent to (2-70):

$$
\vec{H}+\vec{\gamma}_{0} \log |x|-\frac{i}{2} \vec{L}=\vec{E}-\vec{T},
$$

and assume that the antimeromorphic function $\vec{E}$ has a pole of order $\left(\theta_{0}-3\right)$ at the origin, while $\vec{T}=\mathrm{O}\left(|x|^{2-\theta_{0}-\epsilon}\right)$ for all $\epsilon>0$. Exactly as we did in the case $\alpha=\theta_{0}-2$, we obtain

$$
\vec{H}=\mathrm{O}\left(|x|^{2-\theta_{0}-\epsilon}\right) \quad \text { for all } \epsilon>0 .
$$

Calling upon Lemma 2.4 with $k=2$ gives us the alternative representation

$$
-\frac{i}{2} \vec{L}+\vec{H}+\vec{\gamma}_{0} \log |x|=\vec{E}^{1}-\vec{T}^{1},
$$

where $\vec{E}^{1}$ is an antimeromorphic function with a pole at the origin of order at most $\left(\theta_{0}-2\right)$, while $\vec{T}^{1}=\mathrm{O}\left(|x|^{3-\theta_{0}-\epsilon}\right)$. Hence,

$$
\vec{T} \equiv \vec{E}-\vec{E}^{1}+\vec{T}^{1}=-\vec{E}^{1}+\mathrm{O}\left(|x|^{3-\theta_{0}-\epsilon}\right) .
$$


If $\vec{E}^{1}$ had a pole of order $\left(\theta_{0}-2\right)$, then $\vec{T}$ would contain a monomial term of $\bar{x}$, which we have ruled out by hypothesis. Thus, the pole of $\vec{E}^{1}$ has order at most $\left(\theta_{0}-3\right)$. The representation (2-72) then yields

$$
\vec{H}=\mathrm{O}\left(|x|^{3-\theta_{0}-\epsilon}\right) \text { for all } \epsilon>0 .
$$

Finally, calling once more upon Lemma 2.4 with this time $k=3$ gives us the desired representation.

The regularity of the function $\vec{Q}$ is closely tied to that of $\vec{H}$ (and ultimately to that of the Gauss map). A quick inspection of the proof of Proposition 2.5 reveals that, if the local behavior of the mean curvature improves to $\vec{H}=\mathrm{O}\left(|x|^{-\alpha}\right)$ for some $\alpha \in\left\{0, \ldots, \theta_{0}-2\right\}$, then we get the corresponding improvement $\vec{Q}=\mathrm{O}\left(|x|^{-\alpha+1-\epsilon}\right)$ for all $\epsilon>0$. In this case, the order of the pole of the antimeromorphic function $\vec{E}$ is at most $\alpha$. On the other hand, if $\vec{E}$ happens to be regular at the origin, the identity (2-70) shows that the regularity of $\vec{H}$ improves with that of $\vec{Q}$, a condition which, per our previous observation, makes it possible to implement a bootstrapping procedure. The obstruction induced by the singular behavior of the function $\vec{E}$ at the origin is studied in detail in the next section. We view $\vec{E}$ as a string of $m$ complex-valued functions $\left\{E_{j}\right\}_{j=1, \ldots, m}$, all of which are antimeromorphic and may have a pole at the origin of order at most $\left(\theta_{0}-1\right)$. In particular, we define the $\mathbb{N}^{m}$-valued second residue

$$
\vec{\gamma}=\left(\gamma_{1}, \ldots, \gamma_{m}\right) \quad \text { with } \gamma_{j}:=\frac{1}{2 i \pi} \int_{\partial D^{2}} d \log E_{j} .
$$

Remark 2.6. Branched minimal surfaces have vanishing first and second residues. Indeed, if $\vec{H} \equiv \overrightarrow{0}$, from the very definition (1-8) of the first residue, we see that $\vec{\gamma}_{0}=\overrightarrow{0}$. Furthermore, the function $\vec{q}$ introduced in (2-61) is identically vanishing, thereby yielding that $\vec{Q} \equiv \overrightarrow{0}$. According to (2-70), we have $\vec{E}=-\frac{1}{2} i \vec{L}$. But, as seen in (2-9), the function $\vec{L}$ must be constant when $\vec{H} \equiv \overrightarrow{0}$. The function $\vec{E}$ is thus regular, and hence the second residue $\vec{\gamma}$ vanishes.

2E2. How the second residue $\vec{\gamma}$ controls the regularity. We start by defining

$$
\alpha:=\max _{1 \leq j \leq m} \gamma_{j} \in\left\{0, \ldots, \theta_{0}-1\right\} .
$$

Per Proposition 2.5, we may choose $\vec{E}=\vec{E}_{\alpha} \bar{x}^{-\alpha}$ for some constant vector $\vec{E}_{\alpha} \in \mathbb{C}^{m}$. According to Proposition 2.5, we have

$$
\vec{Q}=\mathrm{O}\left(|x|^{1-\alpha-\epsilon}\right) \text { for all } \epsilon>0 .
$$

Because $\vec{L}$ is real-valued, (2-70) yields

$$
\vec{H}+\vec{\gamma}_{0} \log |x|=\Re(\vec{E}-\vec{Q}) .
$$


We define next the two-component vector field $\vec{U}:=(\Re, \Im)\left(\mathrm{e}^{\lambda} \vec{Q}\right)$. As $\partial_{x} \vec{Q}=\vec{q}$, we have ${ }^{19}$

$$
\operatorname{div} \vec{U}=\nabla \lambda \cdot \vec{U}+2 \mathrm{e}^{\lambda} \Re(\vec{q}), \quad \operatorname{curl} \vec{U}=\nabla^{\perp} \lambda \cdot \vec{U}+2 \mathrm{e}^{\lambda} \Im(\vec{q}) .
$$

As $|\nabla \lambda| \lesssim|x|^{-1}$, we use (2-75) along with the estimates (2-62) and (2-74) to find (2-77) $|\operatorname{div} \vec{U}|+|\operatorname{curl} \vec{U}| \lesssim|x|^{-1}|\vec{U}|+\mathrm{e}^{\lambda}|x|^{-\epsilon}|\vec{H}| \lesssim|x|^{\theta_{0}-1-\alpha-\epsilon} \quad$ for all $\epsilon>0$.

With the help of a simple Hodge decomposition, (2-77) along with the fact that $|\vec{U}| \simeq \mathrm{e}^{\lambda}|\vec{Q}|=\mathrm{O}\left(|x|^{\theta_{0}-\epsilon}\right)$ yields

$$
\left|\nabla\left(\mathrm{e}^{\lambda} \vec{Q}\right)\right| \simeq|\nabla \vec{U}| \lesssim|x|^{\theta_{0}-1-\epsilon} \text { for all } \epsilon>0 .
$$

Again since $|\nabla \lambda| \lesssim|x|^{-1}$, the latter shows that

$$
|\nabla \vec{Q}| \lesssim|x|^{-\epsilon} \text { for all } \epsilon>0 .
$$

Putting (2-78) into (2-75), and recalling that $\vec{E}$ is a power function, then yields

$$
|\nabla \vec{H}| \lesssim|x|^{-1-\alpha} .
$$

As $\alpha \leq \theta_{0}-1$, we thus find $\mathrm{e}^{\lambda} \nabla \vec{H} \in L^{2, \infty}$. It is proved as (B-8) in the appendix that the $\Lambda^{m-2}\left(\mathbb{S}^{m-1}\right)$-valued Gauss map $\vec{n}$ satisfies the perturbed harmonic map equation

$$
\Delta \vec{n}-2 \mathrm{e}^{2 \lambda} K \vec{n}=2 \star\left(\nabla^{\perp} \vec{\Phi} \wedge \nabla \vec{H}\right)-2 \star \mathrm{e}^{2 \lambda} \vec{h}_{12} \wedge\left(\vec{h}_{11}-\vec{h}_{22}\right),
$$

where $K$ is the Gauss curvature, whence

$$
|\Delta \vec{n}| \lesssim \mathrm{e}^{\lambda}|\nabla \vec{H}|+|\nabla \vec{n}|^{2} \lesssim|x|^{\theta_{0}-2} \in L^{2, \infty} .
$$

Accordingly, $\nabla^{2} \vec{n} \in L^{2, \infty}$, and in particular $\nabla \vec{n} \in \mathrm{BMO}$.

We have seen in the Introduction that the conformal parameter satisfies

$$
\lambda=\left(\theta_{0}-1\right) \log |x|+u,
$$

where the function $u$ belongs to $W^{2,1}$. More precisely, from the Liouville equation, we know that

$$
-\Delta u=\mathrm{e}^{2 \lambda} K
$$

with $K$ denoting the Gauss curvature. As explained, $\mathrm{e}^{2 \lambda} K$ inherits the regularity of $|\nabla \vec{n}|^{2}$ (as it is made of products of terms of the type $\mathrm{e}^{\lambda} \vec{h}_{i j}$, each of which inherits the regularity of $|\nabla \vec{n}|)$. Owing to (2-81), we thus have $\nabla^{2} u \in \bigcap_{p<\infty} L^{p}$, and in particular

${ }^{19}$ Although the equation for $\vec{Q}$ holds only on $D^{2} \backslash\{0\}$, the system for $\vec{U}$ may easily be extended to the whole unit disk $D^{2}$ owing to the fact that $\vec{U}=\mathrm{O}\left(|x|^{1-\epsilon}\right) \in L^{\infty}$. 
that $\nabla u$ is Hölder continuous. Hence, (2-82) shows that

$$
|\nabla \lambda| \lesssim|x|^{-1}
$$

Furthermore, we may write

$$
2 \mathrm{e}^{2 \lambda}=\left(T_{1}+R_{1}\right)|x|^{2\left(\theta_{0}-1\right)},
$$

where $T_{1}$ is the first-order Taylor polynomial expansion of $2 \mathrm{e}^{2 u} \in C^{1,1-\epsilon}$ (for all $\epsilon>0$ ) near the origin, and $R_{1}$ is the corresponding remainder. Hence

$$
\nabla^{j} R_{1}=\mathrm{O}\left(|x|^{2-j-\epsilon}\right), \quad j \in\{0,1\}, \text { for all } \epsilon>0 .
$$

With the help of (2-75), we write

$$
\Delta \vec{\Phi} \equiv 2 \mathrm{e}^{2 \lambda} \vec{H}=\Delta \vec{\Phi}_{0}+\Delta \vec{\Phi}_{1},
$$

where

$$
\left\{\begin{array}{l}
\Delta \vec{\Phi}_{0}=T_{1}|x|^{2\left(\theta_{0}-1\right)} \Re\left(\vec{E}-\vec{\gamma}_{0} \log |x|\right), \\
\Delta \vec{\Phi}_{1}=-2 \mathrm{e}^{2 \lambda} \Re(\vec{Q})+|x|^{2\left(\theta_{0}-1\right)} R_{1} \Re\left(\vec{E}-\vec{\gamma}_{0} \log |x|\right) .
\end{array}\right.
$$

Since $T_{1}$ and $\vec{E}$ are power functions, we easily obtain via solving explicitly and handling the remainder with Corollary C.3 that

$$
\vec{\Phi}_{0}=\Re\left(\vec{P}_{0}\right)+C_{\alpha}|x|^{2 \theta_{0}} \Re(\vec{E})-\vec{C}|x|^{2 \theta_{0}}\left(\log |x|^{2 \theta_{0}}-4\right)+\vec{\xi}_{0},
$$

where $\vec{P}_{0}$ is a $\mathbb{C}^{m}$-valued holomorphic polynomial of degree at most $\left(2 \theta_{0}-\alpha\right)$, and

$$
C_{\alpha}:=\frac{\mathrm{e}^{2 u(0)}}{2 \theta_{0}\left(\theta_{0}-\alpha\right)} \quad \text { and } \quad \vec{C}:=\frac{\mathrm{e}^{2 u(0)}}{2 \theta_{0}^{3}} \vec{\gamma}_{0} .
$$

The remainder $\vec{\xi}_{0}$ satisfies

$$
\begin{gathered}
\nabla^{j} \vec{\xi}_{0}=\mathrm{O}\left(|x|^{2 \theta_{0}-\alpha+1-j-\epsilon}\right) \quad \text { for all } j \in\{0, \ldots, 2\} \text {, for all } \epsilon>0, \\
\qquad|x|^{2+\alpha-2 \theta_{0}} \nabla^{3} \vec{\xi}_{0} \in \bigcap_{p<\infty} L^{p}
\end{gathered}
$$

To obtain information on $\vec{\Phi}_{1}$, we differentiate once its partial differential equation in each coordinate $x_{1}$ and $x_{2}$, and apply Corollary C.3 to the respective results using (2-85), the fact that $\mathrm{e}^{\lambda} \vec{Q} \in \bigcup_{p<\infty} W^{1, p}$, that $\nabla \lambda=\mathrm{O}\left(|x|^{-1}\right)$, and the fact that $\vec{F}$ is a power function. Without much effort, it ensues that we can write

$$
\vec{\Phi}_{1}=\Re\left(\vec{P}_{1}\right)+\vec{\xi}_{1},
$$

where $\vec{P}_{1}$ is a $\mathbb{C}^{m}$-valued holomorphic polynomial of degree at most $\left(2 \theta_{0}-\alpha\right)$, and the $\mathbb{R}^{m}$-valued function $\vec{\xi}_{1}$ satisfies 


$$
\begin{gathered}
\nabla^{j} \vec{\xi}_{1}=\mathrm{O}\left(|x|^{2 \theta_{0}-\alpha+1-j-\epsilon}\right) \quad \text { for all } j \in\{0, \ldots, 2\}, \text { for all } \epsilon>0, \\
|x|^{2+\alpha-2 \theta_{0}} \nabla^{3} \vec{\xi}_{1} \in \bigcap_{p<\infty} L^{p} .
\end{gathered}
$$

Comparing $\vec{\Phi}_{0}+\vec{\Phi}_{1}$ to the previously found expression (2-21), we deduce

$$
\vec{\Phi}=\Re\left(\vec{A} x^{\theta_{0}}+\vec{B}_{1} x^{\theta_{0}+1}+C_{\alpha}|x|^{2 \theta_{0}} \vec{E}\right)-\vec{C}|x|^{2 \theta_{0}}\left(\log |x|^{2 \theta_{0}}-4\right)+\left(\vec{\xi}_{0}+\vec{\xi}_{1}\right),
$$

where $\vec{B}_{1} \in \mathbb{C}^{m}$ is constant, while $\vec{A}$ is as in Proposition 1.3 .

Note that

$$
\left|\nabla^{j} \vec{\Phi}\right|=\mathrm{O}\left(|x|^{\theta_{0}-j}\right) \quad \text { for all } j \in\{0, \ldots, 2\} .
$$

Suppose next that $\alpha \leq \theta_{0}-2$. Then (2-79) gives

$$
\mathrm{e}^{\lambda} \nabla \vec{H} \in L^{\infty} \text {. }
$$

In turn brought into (2-81), the latter shows that

$$
\nabla^{2} \vec{n} \in \bigcap_{p<\infty} L^{p} .
$$

Accordingly, the function $u$ appearing in (2-82) lies in $C^{2,1-\epsilon}\left(D^{2}\right)$ for all $\epsilon>0$, whence

$$
\nabla^{2} \lambda=\mathrm{O}\left(|x|^{-2}\right) .
$$

When $\alpha \leq \theta_{0}-2$, we have that $|x|^{-1} \mathrm{e}^{\lambda}|\vec{E}| \simeq|x|^{\theta_{0}-2-\alpha} \in L^{\infty}$. Hence (2-74) and (2-75) yield

$$
|x|^{-1} \mathrm{e}^{\lambda} \vec{H} \in L^{\infty} \text {. }
$$

We now need to improve the regularity of $\vec{q}$. Recall that

$$
\vec{q}:=|\vec{H}|^{2} \partial_{x} \vec{\Phi}+2\left(\vec{H} \cdot \vec{H}_{0}\right) \partial_{\bar{x}} \vec{\Phi}
$$

As e $\mathrm{e}^{\lambda} \vec{H}$ and $\mathrm{e}^{\lambda} \vec{H}_{0}$ inherit the regularity of $\nabla \vec{n}$, we find

$$
\begin{aligned}
\left|\nabla\left(\mathrm{e}^{\lambda}\left(\vec{H} \cdot \vec{H}_{0}\right) \partial_{\bar{x}} \vec{\Phi}\right)\right| & \lesssim\left|\mathrm{e}^{\lambda} \vec{H}\right|\left|\nabla^{2} \vec{n}\right|+|\nabla \vec{n}|\left(\mathrm{e}^{\lambda}|\nabla \vec{H}|+\left|\nabla^{2} \vec{\Phi}\right||\vec{H}|\right) \\
& \lesssim|x|\left|\nabla^{2} \vec{n}\right|+|\nabla \vec{n}|+|x|^{-1} \mathrm{e}^{\lambda}|\vec{H}| \in \bigcap_{p<\infty} L^{p},
\end{aligned}
$$

where we have used (2-91), (2-89), (2-88), and (2-90). Exactly in the same fashion, one verifies that

$$
\mathrm{e}^{\lambda}|\vec{H}|^{2} \partial_{x} \vec{\Phi} \in \bigcap_{p<\infty} W^{1, p} .
$$

Together, the latter and (2-92) brought into the definition of $\vec{q}$ show that

$$
\mathrm{e}^{\lambda} \vec{q} \in \bigcap_{p<\infty} W^{1, p} .
$$


We next return to the system (2-76). Proceeding as in (2-77) with the information that $\alpha \leq \theta_{0}-2$, we infer that

$$
\left|\operatorname{div}\left(|x|^{-1} \vec{U}\right)\right|+\left|\operatorname{curl}\left(|x|^{-1} \vec{U}\right)\right| \lesssim|x|^{\theta_{0}-2-\alpha-\epsilon} \lesssim|x|^{-\epsilon} \quad \text { for all } \epsilon>0,
$$

so that $|x|^{-1} \mathrm{e}^{\lambda} \vec{Q} \equiv|x|^{-1} \vec{U}$ is an element of $W^{1, p}$ for all finite $p$. By a similar token, using (2-93), it is not difficult to see that

$$
\left|\nabla^{2} \vec{U}\right| \lesssim\left|\nabla\left(|x|^{-1} \vec{U}\right)\right|+\left|\nabla\left(\mathrm{e}^{\lambda} \vec{q}\right)\right| \in \bigcap_{p<\infty} L^{p} .
$$

Hence, $\mathrm{e}^{\lambda} \vec{Q} \equiv \vec{U} \in \bigcap_{p<\infty} W^{2, p}$. Using that $\nabla \lambda=\mathrm{O}\left(|x|^{-1}\right)$ now gives

$$
|x|^{-1} \mathrm{e}^{\lambda}|\nabla \vec{Q}| \lesssim\left|\nabla\left(|x|^{-1} \mathrm{e}^{\lambda} \vec{Q}\right)\right|+|x|^{-2} \mathrm{e}^{\lambda}|\vec{Q}| \in \bigcap_{p<\infty} L^{p},
$$

where have used that $\alpha \leq \theta_{0}-2$ and $\vec{Q}=\mathrm{O}\left(|x|^{1-\alpha-\epsilon}\right)$ for all $\epsilon<0$. In particular, owing to (2-75), we have

$$
|x|^{-1} \mathrm{e}^{\lambda}\left|\nabla\left(\vec{H}+\vec{\gamma}_{0} \log |x|-\Re(\vec{E})\right)\right| \lesssim|x|^{-1} \mathrm{e}^{\lambda}|\nabla \vec{Q}| \in \bigcap_{p<\infty} L^{p} .
$$

Analogously, using now additionally that $\nabla^{2} \lambda=\mathrm{O}\left(|x|^{-2}\right)$ yields

$$
\mathrm{e}^{\lambda}\left|\nabla^{2} \vec{Q}\right| \lesssim|x|^{-2}|\vec{U}|+\left|\nabla\left(|x|^{-1} \vec{U}\right)\right|+\left|\nabla^{2} \vec{U}\right| .
$$

As we have shown above, each of these terms lies in $L^{p}$ for all finite $p$. Accordingly, differentiating twice (2-75) yields

$$
\mathrm{e}^{\lambda}\left|\nabla^{2}\left(\vec{H}+\vec{\gamma}_{0} \log |x|-\Re(\vec{E})\right)\right| \lesssim \mathrm{e}^{\lambda}\left|\nabla^{2} \vec{Q}\right| \in \bigcap_{p<\infty} L^{p} .
$$

We have pointed out that the function $u$ in (2-82) lies in $C^{2,1-\epsilon}$ for all $\epsilon>0$, owing to the fact that $\vec{n} \in W^{2, p}$ for all $p<\infty$. We may now replace (2-84) by

$$
2 \mathrm{e}^{2 \lambda}=\left(T_{2}+R_{2}\right)|x|^{2\left(\theta_{0}-1\right)},
$$

where $T_{2}$ is the second-order Taylor polynomial expansion of $2 \mathrm{e}^{2 u}$, and $R_{2}$ is the corresponding remainder. Hence

$$
\nabla^{j} R_{2}=\mathrm{O}\left(|x|^{3-j-\epsilon}\right), \quad j \in\{0, \ldots, 2\}, \text { for all } \epsilon>0 .
$$

As before, we write the decomposition

$$
\Delta \vec{\Phi} \equiv 2 \mathrm{e}^{2 \lambda} \vec{H}=\Delta \vec{\Phi}_{0}+\Delta \vec{\Phi}_{1},
$$

with now

$$
\left\{\begin{array}{l}
\Delta \vec{\Phi}_{0}=T_{2}|x|^{2\left(\theta_{0}-1\right)} \Re\left(\vec{E}-\vec{\gamma}_{0} \log |x|\right), \\
\Delta \vec{\Phi}_{1}=-2 \mathrm{e}^{2 \lambda} \Re(\vec{Q})+|x|^{2\left(\theta_{0}-1\right)} R_{2} \Re\left(\vec{E}-\vec{\gamma}_{0} \log |x|\right) .
\end{array}\right.
$$


Since $T_{2}$ and $\vec{E}$ are power functions, we easily obtain via solving explicitly and handling the remainder with Corollary C.3 that

$$
\vec{\Phi}_{0}=\Re\left(\vec{P}_{0}\right)+C_{\alpha}|x|^{2 \theta_{0}} \Re(\vec{E})-\vec{C}|x|^{2 \theta_{0}}\left(\log |x|^{2 \theta_{0}}-4\right)+\vec{\xi}_{0},
$$

where $\vec{P}_{0}$ is a $\mathbb{C}^{m}$-valued holomorphic polynomial of degree at most $\left(2 \theta_{0}-\alpha\right)$, and the constants $C_{\alpha}$ and $\vec{C}$ are as in (2-86). The remainder $\vec{\xi}_{0}$ satisfies

$$
\begin{gathered}
\nabla^{j} \vec{\xi}_{0}=\mathrm{O}\left(|x|^{2 \theta_{0}-\alpha+1-j-\epsilon}\right) \quad \text { for all } j \in\{0, \ldots, 3\}, \text { for all } \epsilon>0, \\
|x|^{3+\alpha-2 \theta_{0}} \nabla^{4} \vec{\xi}_{0} \in \bigcap_{p<\infty} L^{p} .
\end{gathered}
$$

To obtain information on $\vec{\Phi}_{1}$, we differentiate twice its partial differential equation in each coordinate $x_{1}$ and $x_{2}$, and apply Corollary C.3 to the results using (2-95), the fact that $\mathrm{e}^{\lambda} \vec{Q} \in \bigcap_{p<\infty} W^{2, p}$, that $\nabla^{2} \lambda=\mathrm{O}\left(|x|^{-2}\right)$, and the fact that $\vec{E}$ is a power function. Without much effort, it ensues that we can write

$$
\vec{\Phi}_{1}=\Re\left(\vec{P}_{1}\right)+\vec{\xi}_{1},
$$

where $\vec{P}_{1}$ is a $\mathbb{C}^{m}$-valued holomorphic polynomial of degree at most $\left(2 \theta_{0}-\alpha\right)$, and the $\mathbb{R}^{m}$-valued function $\vec{\xi}_{1}$ satisfies

$$
\begin{gathered}
\nabla^{j} \vec{\xi}_{1}=\mathrm{O}\left(|x|^{2 \theta_{0}-\alpha+1-j-\epsilon}\right) \quad \text { for all } j \in\{0, \ldots, 3\}, \text { for all } \epsilon>0, \\
\qquad|x|^{3+\alpha-2 \theta_{0}} \nabla^{4} \vec{\xi}_{1} \in \bigcap_{p<\infty} L^{p} .
\end{gathered}
$$

Comparing $\vec{\Phi}_{0}+\vec{\Phi}_{1}$ to the previously found expression (2-87), we deduce

$$
\begin{aligned}
\vec{\Phi}=\Re\left(\vec{A} z^{\theta_{0}}+\vec{B}_{1} z^{\theta_{0}+1}+\vec{B}_{2} z^{\theta_{0}+2}+C_{\alpha}|x|^{2 \theta_{0}} \vec{E}\right) & \\
& -\vec{C}|x|^{2 \theta_{0}}\left(\log |x|^{2 \theta_{0}}-4\right)+\left(\vec{\xi}_{0}+\vec{\xi}_{1}\right),
\end{aligned}
$$

where $\vec{A}$ and $\vec{B}_{1}$ are as in (2-87), while $\vec{B}_{2} \in \mathbb{C}^{m}$ is a constant.

Note that

$$
\left|\nabla^{j} \vec{\Phi}\right|=\mathrm{O}\left(|x|^{\theta_{0}-j}\right) \quad \text { for all } j \in\{0, \ldots, 3\} \text {. }
$$

Finally, we return to (2-80). Using the previously noted fact that $\mathrm{e}^{\lambda} \vec{h}_{i j}$ inherit the regularity of $\nabla \vec{n}$, along with (2-90), (2-94), (2-97), we now obtain

$$
\begin{aligned}
|\Delta \nabla \vec{n}| & \lesssim\left|\nabla^{2} \vec{n}\right|+|\nabla \vec{n}|^{2}|\nabla \vec{n}|+|\nabla \vec{\Phi}|\left|\nabla^{2} \vec{H}\right|+\left|\nabla^{2} \vec{\Phi}\right||\nabla \vec{H}| \\
& \lesssim\left|\nabla^{2} \vec{n}\right|+|\nabla \vec{n}|^{2}|\nabla \vec{n}|+\mathrm{e}^{\lambda}\left|\nabla^{2} \vec{H}\right|+|x|^{-1} \mathrm{e}^{\lambda}|\nabla \vec{H}| \\
& \simeq|x|^{\theta_{0}-3-\alpha}+\text { terms in } \bigcap_{p<\infty} L^{p} .
\end{aligned}
$$

This shows that $\nabla^{3} \vec{n} \in L^{2, \infty}$ if $\alpha=\theta_{0}-2$. On the other hand, if $\alpha \leq \theta_{0}-3$, we obtain that $\vec{n} \in \bigcap_{p<\infty} W^{3, p}$. We may then start over again the above procedure 
gaining one order of decay at every step. A clear pattern emerges. Repeating finitely many times the steps performed above, one eventually reaches that

$$
\nabla^{\theta_{0}-\alpha+1} \vec{n} \in L^{2, \infty} \text { and thus } \quad \nabla^{\theta_{0}-\alpha} \vec{n} \in \mathrm{BMO} .
$$

Furthermore, for all $j \in\left\{0, \ldots, \theta_{0}-\alpha\right\}$, we have

$$
|x|^{\alpha+j-1} \nabla^{j}\left(\vec{H}+\vec{\gamma}_{0} \log |x|-\Re(\vec{E})\right) \in \bigcap_{p<\infty} L^{p} .
$$

We also obtain a local expansion for the immersion, namely

$$
\vec{\Phi}=\Re\left(\vec{A} x^{\theta_{0}}+\sum_{j=1}^{\theta_{0}-\alpha} \vec{B}_{j} x^{\theta_{0}+j}+C_{\alpha}|x|^{2 \theta_{0}} \vec{E}\right)-\vec{C}|x|^{2 \theta_{0}}\left(\log |x|^{2 \theta_{0}}-4\right)+\vec{\xi}
$$

where $\vec{B}_{j} \in \mathbb{C}^{m}$ are constant vectors, while $\vec{A}$ is as in (2-70). The constants $C_{\alpha}$ and $\vec{C}$ are

$$
C_{\alpha}:=\frac{\mathrm{e}^{2 u(0)}}{2 \theta_{0}\left(\theta_{0}-\alpha\right)} \quad \text { and } \quad \vec{C}:=\frac{\mathrm{e}^{2 u(0)}}{2 \theta_{0}^{3}} \vec{\gamma}_{0} .
$$

The remainder $\vec{\xi}$ satisfies

$$
\begin{gathered}
\nabla^{j} \vec{\xi}=\mathrm{O}\left(|x|^{2 \theta_{0}-\alpha+1-j-\epsilon}\right) \quad \text { for all } j \in\left\{0, \ldots, \theta_{0}-\alpha+1\right\} \text {, for all } \epsilon>0, \\
\qquad|x|^{1-\theta_{0}} \nabla^{\theta_{0}-\alpha+2} \vec{\xi} \in \bigcap_{p<\infty} L^{p}
\end{gathered}
$$

Of course, when $\alpha>0$, the "remainder" term $\vec{\xi}$ in (2-100) dominates the logarithmic term, written here to indicate the presence and the influence of the (modified) first residue $\vec{\gamma}_{0}$ of which it is a multiple.

2E3. When both residues vanish: smoothness of the immersion. This last section is devoted to proving Theorem 1.9. We shall assume that the first and second residues defined respectively in (1-8) and in (2-73) vanish.

When $\theta_{0}=1$, we have seen at the end of Section $2 \mathrm{C}$ that $\nabla \vec{n} \in \mathrm{BMO}$. In the same section, Proposition 2.2 states that $\vec{H} \in W^{1, p}$ for all $p<\infty$. Hence, $\nabla \vec{H} \in L^{p}$ for all finite $p$. On the other hand, when $\theta \geq 2$, we proved in (2-100) that $\nabla^{\theta_{0}} \vec{n} \in \mathrm{BMO}$ and in (2-99) that $|x|^{j-1} \nabla^{j} \vec{H} \in \bigcap_{p<\infty} L^{p}$ for all $j \in\left\{1, \ldots, \theta_{0}\right\}$. Altogether, in all cases, we thus have

$$
\nabla^{\theta_{0}} \vec{n} \in \mathrm{BMO} \quad \text { and } \quad|x|^{j-1} \nabla^{j} \vec{H} \in \bigcap_{p<\infty} L^{p} \quad \text { for all } j \in\left\{1, \ldots, \theta_{0}\right\} .
$$

Observe that (2-100) implies

$$
\left|\nabla^{j} \vec{\Phi}(x)\right| \lesssim|x|^{\theta_{0}-j} \quad \text { for all } j \in\left\{0, \ldots, \theta_{0}\right\} .
$$


Owing to

$$
\left|\nabla^{\theta_{0}-1}\left(\nabla^{\perp} \vec{\Phi} \wedge \nabla \vec{H}\right)\right| \lesssim \sum_{j=1}^{\theta_{0}}\left|\nabla^{j} \vec{H}\right|\left|\nabla^{\theta_{0}-j+1} \vec{\Phi}\right| \lesssim \sum_{j=1}^{\theta_{0}}|x|^{j-1}\left|\nabla^{j} \vec{H}\right|,
$$

whence we find

$$
\nabla^{\perp} \vec{\Phi} \wedge \nabla \vec{H} \in \bigcap_{p<\infty} W^{\theta_{0}-1, p} .
$$

Recall next (B-8) satisfied by the Gauss map, namely

$$
\Delta \vec{n}=2 \star\left(\nabla^{\perp} \vec{\Phi} \wedge \nabla \vec{H}\right)+2 \mathrm{e}^{2 \lambda} K \vec{n}-2 \star \mathrm{e}^{2 \lambda} \vec{h}_{12} \wedge\left(\vec{h}_{11}-\vec{h}_{22}\right) .
$$

As previously noticed, $\mathrm{e}^{\lambda} \vec{h}_{i j}$ inherits the regularity of $|\nabla \vec{n}|$, so that

$$
\mathrm{e}^{2 \lambda} K \vec{n}-2 \star \mathrm{e}^{2 \lambda} \vec{h}_{12} \wedge\left(\vec{h}_{11}-\vec{h}_{22}\right) \in \bigcap_{p<\infty} W^{\theta_{0}-1, p} .
$$

Introducing (2-101) and (2-103) into (2-102) now shows that $\vec{n} \in W^{\theta_{0}+1, p}$ for all $p<\infty$, thereby improving the regularity of $\vec{n}$. It suffices now to repeat the procedure outlined in Section 2E2 and in the above paragraph until reaching that $\vec{n}$ is smooth, from which it immediately follows that the immersion $\vec{\Phi}$ is smooth as well. This concludes the proof of Theorem 1.9.

\section{Appendix A. Notational conventions}

We place an arrow on all letters referring to elements of $\mathbb{R}^{m}$. To simplify the notation, by $\vec{\Phi} \in X\left(D^{2}\right)$ is meant $\vec{\Phi} \in X\left(D^{2}, \mathbb{R}^{m}\right)$ whenever $X$ is a function space. Similarly, we write $\nabla \vec{\Phi} \in X\left(D^{2}\right)$ for $\nabla \vec{\Phi} \in \mathbb{R}^{2} \otimes X\left(D^{2}, \mathbb{R}^{m}\right)$.

Although this custom may seem at first odd, we allow the differential operators classically acting on scalars to act on elements of $\mathbb{R}^{m}$. Thus, for example, $\nabla \vec{\Phi}$ is the element of $\mathbb{R}^{2} \otimes \mathbb{R}^{m}$ that can be written $\left(\partial_{x_{1}} \vec{\Phi}, \partial_{x_{2}} \vec{\Phi}\right)$. If $S$ is a scalar and $\vec{R}$ an element of $\mathbb{R}^{m}$, we let

$$
\begin{aligned}
\vec{R} \cdot \nabla \vec{\Phi} & :=\left(\vec{R} \cdot \partial_{x_{1}} \vec{\Phi}, \vec{R} \cdot \partial_{x_{2}} \vec{\Phi}\right), \\
\nabla^{\perp} S \cdot \nabla \vec{\Phi}: & =\partial_{x_{1}} S \partial_{x_{2}} \vec{\Phi}-\partial_{x_{2}} S \partial_{x_{1}} \vec{\Phi} \\
\nabla^{\perp} \vec{R} \cdot \nabla \vec{\Phi} & :=\partial_{x_{1}} \vec{R} \cdot \partial_{x_{2}} \vec{\Phi}-\partial_{x_{2}} \vec{R} \cdot \partial_{x_{1}} \vec{\Phi} \\
\nabla^{\perp} \vec{R} \wedge \nabla \vec{\Phi} & :=\partial_{x_{1}} \vec{R} \wedge \partial_{x_{2}} \vec{\Phi}-\partial_{x_{2}} \vec{R} \wedge \partial_{x_{1}} \vec{\Phi}
\end{aligned}
$$

Analogous quantities are defined according to the same logic.

Two operations between multivectors are useful. The interior multiplication $L$ maps a pair comprising a $q$-vector $\gamma$ and a $p$-vector $\beta$ to a $(q-p)$-vector. It is defined via

$$
\langle\gamma\llcorner\beta, \alpha\rangle=\langle\gamma, \beta \wedge \alpha\rangle \quad \text { for each }(q-p) \text {-vector } \alpha \text {. }
$$


Let $\alpha$ be a $k$-vector. The first-order contraction operation $\bullet$ is defined inductively through

$$
\alpha \bullet \beta=\alpha\llcorner\beta \quad \text { when } \beta \text { is a } 1 \text {-vector, }
$$

and

$$
\alpha \bullet(\beta \wedge \gamma)=(\alpha \bullet \beta) \wedge \gamma+(-1)^{p q}(\alpha \bullet \gamma) \wedge \beta
$$

when $\beta$ and $\gamma$ are respectively a $p$-vector and a $q$-vector.

\section{Appendix B. Miscellaneous facts}

On the Gauss map. Let $\vec{\Phi}$ be a conformal immersion of the unit disk into $\mathbb{R}^{m}$. For $j \in\{1,2\}$, we let

$$
\vec{e}_{j}:=\mathrm{e}^{-\lambda} \partial_{x_{j}} \vec{\Phi} \quad \text { with } 2 \mathrm{e}^{2 \lambda}=|\nabla \vec{\Phi}|^{2} .
$$

One easily verifies (see details in [Bernard and Rivière 2011b, Section III.2.2]) that

$$
\pi_{T} \nabla \vec{e}_{j}=\left(\nabla^{\perp} \lambda\right) \vec{e}_{j^{\prime}} \quad \text { where }\left(\vec{e}_{1^{\prime}}, \vec{e}_{2^{\prime}}\right):=\left(\vec{e}_{2},-\vec{e}_{1}\right),
$$

where $\pi_{T}$ denotes projection onto the tangent space spanned by $\left\{\vec{e}_{1}, \vec{e}_{2}\right\}$. Moreover,

$$
\pi_{\vec{n}} \nabla \vec{e}_{j} \equiv \mathrm{e}^{-\lambda} \pi_{\vec{n}} \nabla \partial_{j} \vec{\Phi}=: \mathrm{e}^{\lambda}\left(\begin{array}{l}
\vec{h}_{1 j} \\
\vec{h}_{2 j}
\end{array}\right) .
$$

where $\pi_{\vec{n}}$ denotes projection onto the normal space: $\pi_{\vec{n}}=\mathrm{id}-\pi_{T}$. With this notation, the mean curvature vector takes the form

$$
\vec{H}=\frac{1}{2}\left(\vec{h}_{11}+\vec{h}_{22}\right) \text {. }
$$

The (m-2)-vector $\vec{n}$ satisfies $\vec{n}:=\star\left(\vec{e}_{1} \wedge \vec{e}_{2}\right)$. Accordingly, using (B-1), we have

$$
\nabla \vec{n}=\star\left[\left(\pi_{\vec{n}} \nabla \vec{e}_{1}\right) \wedge \vec{e}_{2}+\vec{e}_{1} \wedge\left(\pi_{\vec{n}} \nabla \vec{e}_{2}\right)\right],
$$

so that

$$
\begin{aligned}
& \Delta \vec{n}=\star\left[\operatorname{div}\left(\pi_{\vec{n}} \nabla \vec{e}_{1}\right) \wedge \vec{e}_{2}+\vec{e}_{1} \wedge \operatorname{div}\left(\pi_{\vec{n}} \nabla \vec{e}_{2}\right)\right]+2 \star\left[\pi_{\vec{n}} \nabla \vec{e}_{1} \wedge \pi_{\vec{n}} \nabla \vec{e}_{2}\right] \\
&+\star\left[\pi_{\vec{n}} \nabla \vec{e}_{1} \wedge \pi_{T} \nabla \vec{e}_{2}+\pi_{T} \nabla \vec{e}_{1} \wedge \pi_{\vec{n}} \nabla \vec{e}_{2}\right] .
\end{aligned}
$$

The identities (B-1) yield

$$
\pi_{T} \nabla \vec{e}_{k} \wedge \pi_{\vec{n}} \nabla \vec{e}_{l}=\left(\nabla^{\perp} \lambda\right) \cdot\left(\vec{e}_{k^{\prime}} \wedge \pi_{\vec{n}} \nabla \vec{e}_{l}\right),
$$

and thus

(B-5) $\Delta \vec{n}=\star\left[\operatorname{div}\left(\pi_{\vec{n}} \nabla \vec{e}_{1}\right) \wedge \vec{e}_{2}+\vec{e}_{1} \wedge \operatorname{div}\left(\pi_{\vec{n}} \nabla \vec{e}_{2}\right)\right]+2 \star\left[\pi_{\vec{n}} \nabla \vec{e}_{1} \wedge \pi_{\vec{n}} \nabla \vec{e}_{2}\right]$

$$
+\star\left(\nabla^{\perp} \lambda\right) \cdot\left[\vec{e}_{1} \wedge \pi_{\vec{n}} \nabla \vec{e}_{1}+\vec{e}_{2} \wedge \pi_{\vec{n}} \nabla \vec{e}_{2}\right] .
$$


Next, using the definition of $\vec{e}_{k}$ and again (B-1), we obtain ${ }^{20}$

$$
\begin{aligned}
\operatorname{div} \pi_{\vec{n}} \nabla \vec{e}_{k} & \equiv \pi_{\vec{n}} \operatorname{div} \pi_{\vec{n}} \nabla \vec{e}_{k}+\pi_{T} \operatorname{div} \pi_{\vec{n}} \nabla \vec{e}_{k} \\
& =\pi_{\vec{n}} \operatorname{div} \pi_{\vec{n}} \nabla\left(\mathrm{e}^{-\lambda} \partial_{x_{k}} \vec{\Phi}\right)+\left(\vec{e}_{l} \cdot \operatorname{div} \pi_{\vec{n}} \nabla \vec{e}_{k}\right) \vec{e}_{l} \\
& =\mathrm{e}^{-\lambda} \pi_{\vec{n}} \operatorname{div} \pi_{\vec{n}} \nabla \partial_{x_{k}} \vec{\Phi}-\mathrm{e}^{-\lambda} \pi_{\vec{n}}\left(\nabla \lambda \cdot \nabla \partial_{x_{k}} \vec{\Phi}\right)-\left(\pi_{\vec{n}} \nabla \vec{e}_{l} \cdot \pi_{\vec{n}} \nabla \vec{e}_{k}\right) \vec{e}_{l} \\
& =\mathrm{e}^{-\lambda} \pi_{\vec{n}} \operatorname{div} \pi_{\vec{n}} \nabla \partial_{x_{k}} \vec{\Phi}-\left(\pi_{\vec{n}} \nabla \vec{e}_{l} \cdot \pi_{\vec{n}} \nabla \vec{e}_{k}\right) \vec{e}_{l}-\nabla \lambda \cdot \pi_{\vec{n}} \nabla \vec{e}_{k} .
\end{aligned}
$$

Introducing the latter into (B-5) gives, after a few elementary manipulations,

$$
\begin{aligned}
\Delta \vec{n}=\star \mathrm{e}^{-\lambda}\left[\pi_{\vec{n}} \operatorname{div}\right. & \left.\left(\pi_{\vec{n}} \nabla \partial_{x_{1}} \vec{\Phi}\right) \wedge \vec{e}_{2}+\vec{e}_{1} \wedge \pi_{\vec{n}} \operatorname{div}\left(\pi_{\vec{n}} \nabla \partial_{x_{2}} \vec{\Phi}\right)\right] \\
& -\left[\left|\pi_{\vec{n}} \nabla \vec{e}_{1}\right|^{2}+\left|\pi_{\vec{n}} \nabla \vec{e}_{2}\right|^{2}\right] \star\left(\vec{e}_{1} \wedge \vec{e}_{2}\right)+2 \star\left[\pi_{\vec{n}} \nabla \vec{e}_{1} \wedge \pi_{\vec{n}} \nabla \vec{e}_{2}\right] \\
& +\star\left(\nabla^{\perp} \lambda\right) \cdot\left[\vec{e}_{1} \wedge \pi_{\vec{n}}\left(\nabla \vec{e}_{1}-\nabla^{\perp} \vec{e}_{2}\right)-\pi_{\vec{n}}\left(\nabla^{\perp} \vec{e}_{1}+\nabla \vec{e}_{2}\right) \wedge \vec{e}_{2}\right] .
\end{aligned}
$$

Owing to (B-2) and (B-4), we find

$$
\begin{aligned}
\Delta \vec{n}+|\nabla \vec{n}|^{2} \vec{n}= & \star \mathrm{e}^{-\lambda}\left[\pi_{\vec{n}} \operatorname{div}\left(\pi_{\vec{n}} \nabla \partial_{x_{1}} \vec{\Phi}\right) \wedge \vec{e}_{2}+\vec{e}_{1} \wedge \pi_{\vec{n}} \operatorname{div}\left(\pi_{\vec{n}} \nabla \partial_{x_{2}} \vec{\Phi}\right)\right] \\
& +2 \star \mathrm{e}^{-2 \lambda}\left[\pi_{\vec{n}} \nabla \partial_{x_{1}} \vec{\Phi} \wedge \pi_{\vec{n}} \nabla \partial_{x_{2}} \vec{\Phi}\right]+2 \star \mathrm{e}^{\lambda} \vec{H} \wedge\left[\partial_{x_{2}} \lambda \vec{e}_{1}-\partial_{x_{1}} \lambda \vec{e}_{2}\right] .
\end{aligned}
$$

Equivalently,

$$
\begin{aligned}
\Delta \vec{n}+|\nabla \vec{n}|^{2} \vec{n}=\star \vec{e}_{1} \wedge \mathrm{e}^{-\lambda} & {\left[\pi_{\vec{n}} \operatorname{div}\left(\pi_{\vec{n}} \nabla \partial_{x_{2}} \vec{\Phi}\right)-2 \mathrm{e}^{2 \lambda} \vec{H} \partial_{x_{2}} \lambda\right] } \\
& -\star \vec{e}_{2} \wedge \mathrm{e}^{-\lambda}\left[\pi_{\vec{n}} \operatorname{div}\left(\pi_{\vec{n}} \nabla \partial_{x_{1}} \vec{\Phi}\right)-2 \mathrm{e}^{2 \lambda} \vec{H} \partial_{x_{1}} \lambda\right] \\
& +2 \star\left[\pi_{\vec{n}} \nabla \vec{e}_{1} \wedge \pi_{\vec{n}} \nabla \vec{e}_{2}\right] .
\end{aligned}
$$

Moreover, (B-1) gives $\pi_{T} \nabla \partial_{x_{j}} \vec{\Phi}=\nabla\left(\mathrm{e}^{\lambda}\right) \vec{e}_{j}+\nabla^{\perp}\left(\mathrm{e}^{\lambda}\right) \vec{e}_{j^{\prime}}$. Hence, calling upon (B-2) implies

$$
\pi_{\vec{n}} \operatorname{div} \pi_{T} \nabla \partial_{x_{j}} \vec{\Phi}=\nabla\left(\mathrm{e}^{\lambda}\right) \cdot \pi_{\vec{n}} \nabla \vec{e}_{j}+\nabla^{\perp}\left(\mathrm{e}^{\lambda}\right) \cdot \pi_{\vec{n}} \nabla \vec{e}_{j^{\prime}}=\vec{H} \partial_{x_{j}} \mathrm{e}^{2 \lambda},
$$

and thus, as $\Delta \vec{\Phi}=2 \mathrm{e}^{2 \lambda} \vec{H}$,

$\pi_{\vec{n}} \operatorname{div} \pi_{\vec{n}} \nabla \partial_{x_{j}} \vec{\Phi} \equiv \pi_{\vec{n}} \partial_{x_{j}} \Delta \vec{\Phi}-\pi_{\vec{n}} \operatorname{div} \pi_{T} \nabla \partial_{x_{j}} \vec{\Phi}=2 \pi_{\vec{n}} \partial_{x_{j}}\left(\mathrm{e}^{2 \lambda} \vec{H}\right)-\vec{H} \partial_{x_{j}} \mathrm{e}^{2 \lambda}$.

The interested reader will note that this equation is equivalent to the CodazziMainardi identities. Substituted into (B-6), the latter gives

$$
\begin{aligned}
\Delta \vec{n}+|\nabla \vec{n}|^{2} \vec{n} & =2 \star\left(\partial_{x_{1}} \vec{\Phi} \wedge \pi_{\vec{n}} \partial_{x_{2}} \vec{H}-\partial_{x_{2}} \vec{\Phi} \wedge \pi_{\vec{n}} \partial_{x_{1}} \vec{H}\right)+2 \star\left[\pi_{\vec{n}} \nabla \vec{e}_{1} \wedge \pi_{\vec{n}} \nabla \vec{e}_{2}\right] \\
& =2 \star\left(\nabla^{\perp} \vec{\Phi} \wedge \pi_{\vec{n}} \nabla \vec{H}\right)-2 \star \mathrm{e}^{2 \lambda} \vec{h}_{12} \wedge\left(\vec{h}_{11}-\vec{h}_{22}\right) .
\end{aligned}
$$

One also notes from (B-2) and the fact that $\vec{H}$ is normal that

$$
\pi_{T} \partial_{x_{j}} \vec{H} \equiv\left\langle\vec{e}_{k}, \partial_{x_{j}} \vec{H}\right\rangle \vec{e}_{k}=-\mathrm{e}^{\lambda}\left(\vec{H} \cdot \vec{h}_{j k}\right) \vec{e}_{k},
$$

${ }^{20}$ Implicit summations over repeated indices are understood. 
whence

$$
\nabla^{\perp} \vec{\Phi} \wedge \pi_{T} \nabla \vec{H} \equiv \partial_{x_{1}} \vec{\Phi} \wedge \pi_{T} \partial_{x_{2}} \vec{H}-\partial_{x_{2}} \vec{\Phi} \wedge \pi_{T} \partial_{x_{1}} \vec{H}=-2 \mathrm{e}^{2 \lambda}|\vec{H}|^{2}(\star \vec{n}) .
$$

Equation (B-7) may thus be recast as

$$
\Delta \vec{n}+|\nabla \vec{n}|^{2} \vec{n}=2 \star\left(\nabla^{\perp} \vec{\Phi} \wedge \nabla \vec{H}\right)+4 \mathrm{e}^{2 \lambda}|\vec{H}|^{2} \vec{n}-2 \star \mathrm{e}^{2 \lambda} \vec{h}_{12} \wedge\left(\vec{h}_{11}-\vec{h}_{22}\right) .
$$

Finally, since

$$
|\nabla \vec{n}|^{2}-4 \mathrm{e}^{2 \lambda}|\vec{H}|^{2}=-2 \mathrm{e}^{2 \lambda} K,
$$

where $K$ is the Gauss curvature, we obtain

$$
\Delta \vec{n}-2 \mathrm{e}^{2 \lambda} K \vec{n}=2 \star\left(\nabla^{\perp} \vec{\Phi} \wedge \nabla \vec{H}\right)-2 \star \mathrm{e}^{2 \lambda} \vec{h}_{12} \wedge\left(\vec{h}_{11}-\vec{h}_{22}\right) .
$$

Conservative conformal Willmore system. We establish in this section a few general identities. As before, we let $\vec{\Phi}$ be a (smooth) conformal immersion of the unit disk into $\mathbb{R}^{m}$, and set $\vec{e}_{j}:=\mathrm{e}^{-\lambda} \partial_{x_{j}} \vec{\Phi}$, where $\lambda$ is the conformal parameter. Since $\vec{\Phi}$ is conformal, $\left\{\vec{e}_{1}, \vec{e}_{2}\right\}$ forms an orthonormal basis of the tangent space. As $\vec{n}=\star\left(\vec{e}_{1} \wedge \vec{e}_{2}\right)$, if $\vec{V}$ is a 1-vector, we find

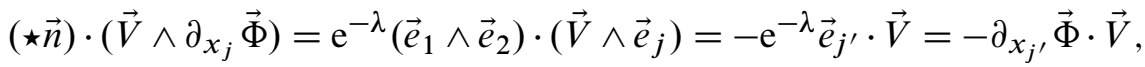

where

$$
\left(\vec{e}_{1^{\prime}}, \vec{e}_{2^{\prime}}\right):=\left(\vec{e}_{2},-\vec{e}_{1}\right)
$$

Hence,

$$
(\star \vec{n}) \cdot(\vec{V} \wedge \nabla \vec{\Phi})=\vec{V} \cdot \nabla^{\perp} \vec{\Phi}, \quad(\star \vec{n}) \cdot\left(\vec{V} \wedge \nabla^{\perp} \vec{\Phi}\right)=-\vec{V} \cdot \nabla \vec{\Phi}
$$

We choose next an orthonormal basis $\left\{\vec{n}_{\alpha}\right\}_{\alpha=1}^{m-2}$ of the normal space such that $\left\{\vec{e}_{1}, \vec{e}_{2}, \vec{n}_{1}, \ldots, \vec{n}_{m-2}\right\}$ is a positive oriented orthonormal basis of $\mathbb{R}^{m}$.

Recalling the definition of the interior multiplication operator $L$ given in Appen$\operatorname{dix} \mathrm{A}$, it is not hard to obtain

$$
(\star \vec{n})\left\llcorner\vec{e}_{j}=\left(\vec{e}_{1} \wedge \vec{e}_{2}\right)\left\llcorner\vec { e } _ { j } = \delta _ { j 2 } \vec { e } _ { 1 } - \delta _ { j 1 } \vec { e } _ { 2 } \quad \text { and } \quad ( \star \vec { n } ) \left\llcorner\vec{n}_{\alpha}=0 .\right.\right.\right.
$$

Hence,

$(\star \vec{n}) \bullet\left(\vec{e}_{j} \wedge \vec{n}_{\alpha}\right) \equiv\left((\star \vec{n})\left\llcorner\vec{e}_{j}\right) \wedge \vec{n}_{\alpha}+\left((\star \vec{n})\left\llcorner\vec{n}_{\alpha}\right) \wedge \vec{e}_{j}=\delta_{j 2} \vec{e}_{1} \wedge \vec{n}_{\alpha}-\delta_{j 1} \vec{e}_{2} \wedge \vec{n}_{\alpha}\right.\right.$.

Moreover, we have trivially

$$
(\star \vec{n}) \bullet\left(\vec{e}_{j} \wedge \vec{e}_{k}\right)= \pm(\star \vec{n}) \bullet(\star \vec{n})=0 .
$$

From this one easily deduces that, for every 1 -vector $\vec{V}$,

(B-10) $(\star \vec{n}) \bullet(\vec{V} \wedge \nabla \vec{\Phi})=\pi_{\vec{n}} \vec{V} \wedge \nabla^{\perp} \vec{\Phi}, \quad(\star \vec{n}) \bullet\left(\vec{V} \wedge \nabla^{\perp} \vec{\Phi}\right)=-\pi_{\vec{n}} \vec{V} \wedge \nabla \vec{\Phi}$. 
We have furthermore

$$
\left(\vec{V} \wedge \vec{e}_{j}\right) \bullet \vec{e}_{i}=\left(\vec{e}_{i} \cdot \vec{V}\right) \vec{e}_{j}-\delta_{i j} \vec{V} .
$$

From this, and $\vec{e}_{i}:=\mathrm{e}^{-\lambda} \partial_{x^{i}} \vec{\Phi}$, it follows that whenever $\vec{V}=V^{i} \vec{e}_{i}+V^{\alpha} \vec{n}_{\alpha}$ then

$$
\left\{\begin{aligned}
\left(\vec{V} \wedge \nabla^{\perp} \vec{\Phi}\right) \cdot \nabla^{\perp} \vec{\Phi} & =\mathrm{e}^{2 \lambda}\left(\pi_{T} \vec{V}-2 \vec{V}\right), \\
(\vec{V} \wedge \nabla \vec{\Phi}) \cdot \nabla^{\perp} \vec{\Phi} & =\mathrm{e}^{2 \lambda}\left(V^{2} \vec{e}_{1}-V^{1} \vec{e}_{2}\right) \equiv(\vec{V} \cdot \nabla \vec{\Phi}) \cdot \nabla^{\perp} \vec{\Phi} .
\end{aligned}\right.
$$

We are now sufficiently geared to prove:

Lemma B.1. Let $\vec{\Phi}$ be a smooth conformal immersion of the unit disk into $\mathbb{R}^{m}$ with corresponding mean curvature vector $\vec{H}$, and let $\vec{L}$ be a 1-vector. We define $A \in \mathbb{R}^{2} \otimes \bigwedge^{0}\left(\mathbb{R}^{m}\right)$ and $\vec{B} \in \mathbb{R}^{2} \otimes \bigwedge^{2}\left(\mathbb{R}^{m}\right)$ via

$$
A=\vec{L} \cdot \nabla \vec{\Phi}, \quad \vec{B}=\vec{L} \wedge \nabla \vec{\Phi}+2 \vec{H} \wedge \nabla^{\perp} \vec{\Phi} .
$$

Then the following identities hold:

$$
A=-(\star \vec{n}) \cdot \vec{B}^{\perp}, \quad \vec{B}=-(\star \vec{n}) \cdot \vec{B}^{\perp}+(\star \vec{n}) A^{\perp},
$$

where $\star \vec{n}:=\left(\partial_{x_{1}} \vec{\Phi} \wedge \partial_{x_{2}} \vec{\Phi}\right) /\left|\partial_{x_{1}} \vec{\Phi} \wedge \partial_{x_{2}} \vec{\Phi}\right|$.

Moreover, we have

$$
2 \Delta \vec{\Phi}=A \cdot \nabla^{\perp} \vec{\Phi}-\vec{B} \cdot \nabla^{\perp} \vec{\Phi}
$$

Proof. The identities (B-9) give immediately (recall that $\vec{H}$ is a normal vector, so that $\vec{H} \cdot \nabla^{\perp} \vec{\Phi}=0$ ) the required

$$
(\star \vec{n}) \cdot \vec{B}^{\perp}=-\vec{L} \cdot \nabla \vec{\Phi}+2 \vec{H} \cdot \nabla^{\perp} \vec{\Phi}=-\vec{L} \cdot \nabla \vec{\Phi}=-A .
$$

Analogously, the identities (B-10) give (again, $\vec{H}$ is normal, so $\pi_{\vec{n}} \vec{H}=\vec{H}$ )

$$
\begin{aligned}
(\star \vec{n}) \cdot \vec{B}^{\perp} & =-\pi_{\vec{n}} \vec{L} \wedge \nabla \vec{\Phi}-2 \vec{H} \wedge \nabla^{\perp} \vec{\Phi}=-\vec{B}+\pi_{T} \vec{L} \wedge \nabla \vec{\Phi} \\
& =-\vec{B}+\mathrm{e}^{\lambda}\left(\left(\vec{L} \cdot \vec{e}_{1}\right) \vec{e}_{1}+\left(\vec{L} \cdot \vec{e}_{2}\right) \vec{e}_{2}\right) \wedge\left(\begin{array}{c}
\vec{e}_{1} \\
\vec{e}_{2}
\end{array}\right)=-\vec{B}+\mathrm{e}^{\lambda}\left(\begin{array}{c}
-\vec{L} \cdot \vec{e}_{2} \\
+\vec{L} \cdot \vec{e}_{1}
\end{array}\right) \vec{e}_{1} \wedge \vec{e}_{2} \\
& =-\vec{B}+\left(\vec{L} \cdot \nabla^{\perp} \vec{\Phi}\right)(\star \vec{n})=-\vec{B}+(\star \vec{n}) A^{\perp},
\end{aligned}
$$

which is the second equality in (B-12).

In order to prove (B-13), we will use (B-11). Namely, since $\vec{H}=H^{\alpha} \vec{n}_{\alpha}$, we find

$$
\vec{B} \bullet \nabla^{\perp} \vec{\Phi}=(\vec{L} \cdot \nabla \vec{\Phi}) \cdot \nabla^{\perp} \vec{\Phi}-4 \mathrm{e}^{2 \lambda} \vec{H}=A \cdot \nabla^{\perp} \vec{\Phi}-4 \mathrm{e}^{2 \lambda} \vec{H}
$$

Hence,

$$
\vec{B} \cdot \nabla^{\perp} \vec{\Phi}-A \cdot \nabla^{\perp} \vec{\Phi}=-4 \mathrm{e}^{2 \lambda} \vec{H} .
$$

Finally, there remains to recall that $\Delta \vec{\Phi}=2 \mathrm{e}^{2 \lambda} \vec{H}$ to reach the desired identity. 
We choose now

$$
A=\nabla S-\nabla^{\perp} g \quad \text { and } \quad \vec{B}=\nabla \vec{R}-\nabla^{\perp} \vec{G},
$$

where $S$ and $g$ are scalars, while $\vec{R}$ and $\vec{G}$ are 2-vectors. Then Lemma B.1 yields

$$
\left\{\begin{array}{l}
\nabla S=-(\star \vec{n}) \cdot\left(\nabla^{\perp} \vec{R}+\nabla \vec{G}\right)+\nabla^{\perp} g, \\
\nabla \vec{R}=-(\star \vec{n}) \cdot\left(\nabla^{\perp} \vec{R}+\nabla \vec{G}\right)+(\star \vec{n})\left(\nabla^{\perp} S+\nabla g\right)+\nabla^{\perp} \vec{G},
\end{array}\right.
$$

thereby giving

$$
\left\{\begin{array}{l}
-\Delta S=\nabla(\star \vec{n}) \cdot \nabla^{\perp} \vec{R}+\operatorname{div}((\star \vec{n}) \cdot \nabla \vec{G}), \\
-\Delta \vec{R}=\nabla(\star \vec{n}) \cdot \nabla^{\perp} \vec{R}-\nabla(\star \vec{n}) \cdot \nabla^{\perp} S+\operatorname{div}((\star \vec{n}) \bullet \nabla \vec{G}-\star \vec{n} \nabla g) .
\end{array}\right.
$$

Furthermore, we have

$$
2 \Delta \vec{\Phi}=\left(\nabla S-\nabla^{\perp} g\right) \cdot \nabla^{\perp} \vec{\Phi}-\left(\nabla \vec{R}-\nabla^{\perp} \vec{G}\right) \cdot \nabla^{\perp} \vec{\Phi} .
$$

\section{Appendix C. Nonlinear and weighted elliptic results}

Proposition C.1. Let $u \in W^{1,2}\left(B_{1}(0)\right) \cap C^{2}\left(B_{1}(0) \backslash\{0\}\right)$ satisfy the equation

$$
-\Delta u=\nabla b \cdot \nabla^{\perp} u+\operatorname{div}(b \nabla f) \quad \text { on } B_{1}(0),
$$

where $f \in W_{0}^{2,(2, \infty)}\left(B_{1}(0)\right)$, and moreover

$$
b \in W^{1,2} \cap L^{\infty}\left(B_{1}(0)\right) \text { with }\|\nabla b\|_{L^{2}\left(B_{1}(0)\right)}<\varepsilon_{0}
$$

for some $\varepsilon_{0}$ chosen to be "small enough". Then

$$
\nabla u \in L^{p}\left(B_{1 / 4}(0)\right) \text { for some } p>2 \text {. }
$$

Proof. Before delving into the proof of the statement, one important remark is in order. Let $D$ be any disk included (properly or not) in $B_{1}(0)$. From the very definition of the space $L^{2, \infty}$ (see [Tartar 2007]), we have

$$
\|\Delta f\|_{L^{1}(D)} \leq|D|^{\frac{1}{2}}\|\Delta f\|_{L^{2, \infty}(D)} \lesssim|D|^{\frac{1}{2}}\left\|\nabla^{2} f\right\|_{L^{2, \infty}(D)} .
$$

Moreover, an embedding result of [Tartar 2007] states that $\nabla f$ has bounded mean oscillations, whence in particular

$$
\|\nabla f\|_{L^{2}(D)} \lesssim|D|^{\frac{1}{2}-\epsilon} \quad \text { for all } \epsilon>0 .
$$

These inequalities shall be helpful in the sequel.

We now return to the proof of the proposition. Let us fix some point $x_{0} \in B_{1 / 2}(0)$ and some radius $\sigma \in\left(0, \frac{1}{2}\right)$, and we let $k \in(0,1)$. Note that $B_{k \sigma}\left(x_{0}\right)$ is properly 
contained in $B_{1}(0)$. To reach the desired result, we decompose the solution to (C-1) as the sum $u=u_{0}+u_{1}$, where

$$
\left\{\begin{aligned}
-\Delta u_{0} & =\operatorname{div}(b \nabla f), & -\Delta u_{1} & =\nabla b \cdot \nabla^{\perp} u & & \text { in } B_{\sigma}\left(x_{0}\right), \\
u_{0} & =u, & u_{1} & =0 & & \text { on } \partial B_{\sigma}\left(x_{0}\right) .
\end{aligned}\right.
$$

Accounting for the hypotheses (C-2) and (C-4) in standard elliptic estimates (see [Almeida 1995, Proposition 4]) yields

$$
\begin{aligned}
\left\|\nabla u_{0}\right\|_{L^{2}\left(B_{k \sigma}\left(x_{0}\right)\right)} & \lesssim\|b \nabla f\|_{L^{2}\left(B_{k \sigma}\left(x_{0}\right)\right)}+k\|\nabla u\|_{L^{2}\left(B_{\sigma}\left(x_{0}\right)\right)} \\
& \lesssim(k \sigma)^{1-\epsilon}+k\|\nabla u\|_{L^{2}\left(B_{\sigma}\left(x_{0}\right)\right)},
\end{aligned}
$$

up to some unimportant multiplicative constants. On the other hand, applying Wente's inequality (see [Hélein 1996, Theorem 3.4.1]) gives

$$
\text { (C-6) } \begin{aligned}
\left\|\nabla u_{1}\right\|_{L^{2}\left(B_{k \sigma}\left(x_{0}\right)\right)} & \leq\left\|\nabla u_{1}\right\|_{L^{2}\left(B_{\sigma}\left(x_{0}\right)\right)} \lesssim\|\nabla b\|_{L^{2}\left(B_{\sigma}\left(x_{0}\right)\right)}\|\nabla u\|_{L^{2}\left(B_{\sigma}\left(x_{0}\right)\right)} \\
& \leq \varepsilon_{0}\|\nabla u\|_{L^{2}\left(B_{\sigma}\left(x_{0}\right)\right)}
\end{aligned}
$$

again up to some multiplicative constant without bearing on the sequel. Hence, combining (C-5) and (C-6), we obtain the estimate

$$
\begin{aligned}
\|\nabla u\|_{L^{2}\left(B_{k \sigma}\left(x_{0}\right)\right)} & \leq\left\|\nabla u_{0}\right\|_{L^{2}\left(B_{k \sigma}\left(x_{0}\right)\right)}+\left\|\nabla u_{1}\right\|_{L^{2}\left(B_{k \sigma}\left(x_{0}\right)\right)} \\
& \lesssim\left(k+\varepsilon_{0}\right)\|\nabla u\|_{L^{2}\left(B_{\sigma}\left(x_{0}\right)\right)}+(k \sigma)^{1-\epsilon} .
\end{aligned}
$$

Because $\varepsilon_{0}$ and $\epsilon$ are small adjustable parameters, we may always choose $k$ so as to arrange for $\left(k+\varepsilon_{0}\right)$ to be less than 1. A standard "controlled-growth" argument (see, e.g., [Hélein 1996, Lemma 3.5.11]) enables us to conclude that there exists some $\beta \in(0,1)$ for which

$$
\|\nabla u\|_{L^{2}\left(B_{\sigma}(x)\right)} \leq C_{0} \sigma^{\beta} \quad \text { for all } \sigma \in\left(0, \frac{1}{2}\right), x \in B_{1 / 2}(0),
$$

and for some constant $C_{0}$.

With the help of the Poincaré inequality, this estimate may be used to show that $u$ is locally Hölder continuous. We are however interested in another implication of (C-7). Consider the maximal function

$$
M_{2-\beta} g(x):=\sup _{\sigma>0} \sigma^{-\beta} \int_{B_{\sigma}(x)}|g(y)| d y .
$$

We recast $(\mathrm{C}-1)$ in the form

$$
-\Delta u=b \Delta f+\nabla b \cdot\left(\nabla^{\perp} u+\nabla f\right) .
$$


Calling upon (C-2)-(C-4) and upon the estimate (C-7), we derive that, for $x \in$ $B_{1 / 2}(0)$, we have

(C-9) $\quad M_{2-\beta}\left(\chi_{B_{1 / 2}(0)} \Delta u\right)(x)$

$$
\begin{aligned}
& \leq\|b\|_{L^{\infty}\left(B_{1}(0)\right)} \sup _{0<\sigma<1 / 2} \sigma^{-\beta}\|\Delta f\|_{L^{1}\left(B_{\sigma}(x)\right)} \\
& \quad+\|\nabla b\|_{L^{2}\left(B_{1}(0)\right)} \sup _{0<\sigma<1 / 2} \sigma^{-\beta}\left(\|\nabla u\|_{L^{2}\left(B_{\sigma}(x)\right)}+\|\nabla f\|_{L^{2}\left(B_{\sigma}(x)\right)}\right) \\
& \lesssim \sup _{0<\sigma<1 / 2} \sigma^{-\beta+1}+\varepsilon_{0} \sup _{0<\sigma<1 / 2}\left(\sigma^{-\beta+\beta}+\sigma^{-\beta+1-\epsilon}\right)<\infty
\end{aligned}
$$

for all $0<\epsilon \leq 1-\beta$. Moreover, it is clear that $\Delta u$ is integrable on $B_{1 / 2}(0)$. We may thus use Proposition 3.2 from [Adams 1975] ${ }^{21}$ to deduce that

$$
\frac{1}{|x|} * \chi_{B_{1 / 2}(0)} \Delta u \in L^{r, \infty}\left(B_{1 / 2}(0)\right) \quad \text { with } r:=\frac{2-\beta}{1-\beta}>2 .
$$

A classical estimate about Riesz kernels states we have in general

$$
\left.|\nabla u|(y) \lesssim \frac{1}{|x|} * \chi_{B_{1 / 2}(0)} \Delta u+C \quad \text { for all } y \in B_{1 / 4}(0)\right),
$$

where $C$ is a constant depending on the $C^{1}$-norm of $u$ on $\partial B_{1 / 2}(0)$, hence finite by hypothesis. It follows in particular that, as announced,

$$
\nabla u \in L^{p}\left(B_{1 / 4}(0)\right) \text { for all } p<r .
$$

Proposition C.2. Let $u \in C^{2}\left(B_{1}(0) \backslash\{0\}\right)$ solve

$$
\Delta u(x)=\mu(x) f(x) \quad \text { in } B_{1}(0),
$$

where $f \in L^{p}\left(B_{1}(0)\right)$ for some $p>2$. The weight $\mu$ satisfies

$$
|\mu(x)| \simeq|x|^{a} \text { for some } a \in \mathbb{N} .
$$

Then:

(i) We have 22

$$
\nabla u(x)=P(\bar{x})+|\mu(x)| T(x),
$$

where $P(\bar{x})$ is a complex-valued polynomial of degree at most $a$, and near the origin $T(x)=\mathrm{O}\left(|x|^{1-2 / p-\epsilon}\right)$ for every $\epsilon>0$.

${ }^{21}$ Namely, $\left\||x|^{-1} * g\right\|_{L^{r, \infty}}^{r} \lesssim\left\|M_{2-\beta} g\right\|_{L^{\infty}}^{1-1 / r}\|g\|_{L^{1}}^{1 / r}$ for $r=(2-\beta) /(1-\beta)$ and $\beta \in(0,1)$.

${ }^{22} \bar{x}$ is the complex conjugate of $x$. We parametrize $B_{1}(0)$ by $x=x_{1}+i x_{2}$, and then $\bar{x}:=x_{1}-i x_{2}$. In this notation, $\nabla u$ in $(\mathrm{C}-12)$ is understood as $\partial_{x_{1}} u+i \partial_{x_{2}} u$. 
(ii) Furthermore, if $\mu \in C^{1}\left(B_{1}(0) \backslash\{0\}\right)$, if $a \neq 0$, and if

$$
|x|^{1-a} \nabla \mu(x) \in L^{\infty}\left(B_{1}(0)\right),
$$

we have

$$
\nabla^{2} u(x)=\nabla P(\bar{x})+|\mu(x)| Q(x),
$$

where $P$ is as in (i), and

$$
Q \in L^{p-\epsilon}\left(B_{1}(0), \mathbb{C}^{2}\right) \text { for all } \epsilon>0 .
$$

As a $(2 \times 2)$ real-valued matrix, $Q$ satisfies in addition

$$
\operatorname{Tr} Q \in L^{p}\left(B_{1}(0)\right) \text {. }
$$

Naturally, if $a=0$, the standard Calderon-Zygmund theorem yields that $u \in W^{2, p}\left(B_{1}(0)\right)$. The hypothesis $(\mathrm{C}-13)$ becomes unnecessary, and $(\mathrm{C}-14)$ holds with $P$ being constant and $\epsilon=0$.

Proof. Using Green's formula for the Laplacian, an exact expression for the solution $u$ may be found and used to obtain, for all $x \in B_{1}(0)$ and with $\vec{v}$ the outer normal unit vector to the boundary of $B_{1}(0)$,

$$
\begin{aligned}
& \nabla u(x)=\frac{1}{2 \pi} \int_{\partial B_{1}(0)}\left(\frac{x-y}{|x-y|^{2}} \partial_{\vec{v}} u(y)-u(y) \partial_{\vec{v}} \frac{x-y}{|x-y|^{2}}\right) d \sigma(y) \\
&=: \frac{1}{2 \pi} \int_{B_{1}(0)} \frac{x-y}{|x-y|^{2}} \mu(y) f(y) d y \\
& J_{0}(x)+J_{1}(x) .
\end{aligned}
$$

Without loss of generality, and to avoid notational clutter, because $u$ is twice differentiable away from the origin, we shall henceforth assume that $|x|<1 / 2$.

We will estimate separately $J_{0}$ and $J_{1}$, and open the discussion by noting that, when $|y|>|x|$, we have the expansion

$$
\frac{x-y}{|x-y|^{2}}=-\sum_{m \geq 0} P^{m}(x, y) \quad \text { with } P^{m}(x, y):=\bar{x}^{m} \bar{y}^{-(m+1)} .
$$

Hence, we deduce the identity

$$
\text { (C-16) } \begin{aligned}
J_{0}(x) & =-\frac{1}{2 \pi} \sum_{m \geq 0} \int_{\partial B_{1}(0)}\left[P^{m}(x, y) \partial_{\vec{v}} u(y)-u(y) \partial_{\vec{v}} P^{m}(x, y)\right] d S(y) \\
& =-\frac{1}{2 \pi} \sum_{m \geq 0} \bar{x}^{m} \int_{0}^{2 \pi}\left[(m+1) u\left(\mathrm{e}^{i \varphi}\right)-\left(\partial_{\vec{v}} u\right)\left(\mathrm{e}^{i \varphi}\right)\right] \mathrm{e}^{i(m+1) \varphi} d \varphi \\
& =\sum_{m \geq 0} C_{m} \bar{x}^{m}
\end{aligned}
$$


where the $C_{m}$ are (complex-valued) constants depending only on the $C^{1}$-norm of $u$ along $\partial B_{1}(0)$. As $u$ is continuously differentiable on the boundary of the unit disk by hypothesis, and $|x|<1$, it is clear that $\left|J_{0}(x)\right|$ is bounded above by some constant $C$ for all $x \in B_{1}(0)$. Since $\left|C_{m}\right|$ grows sublinearly in $m$, we can surely find two constants $\gamma$ and $\delta$ such that

$$
\left|C_{m}\right|<\gamma \delta^{m} \quad \text { for all } m \geq 0 .
$$

Hence, when $|x| \leq R<\delta^{-1}$, we have

$$
\left|\sum_{m \geq a+1} C_{m} \bar{x}^{m}\right| \leq \gamma \delta^{a+1}|x|^{a+1} \sum_{m \geq 0}(\delta R)^{m} \lesssim|x|^{a+1} .
$$

And because $J_{0}$ is bounded, when $R<|x|<1$, we find some large enough constant $K=K(C, a, \gamma, \delta)$ such that

$$
\begin{aligned}
\left|\sum_{m \geq a+1} C_{m} \bar{x}^{m}\right| & \leq\left|J_{0}(x)\right|+\sum_{0 \leq m \leq a} C_{m}|x|^{m} \leq C+(a+1) \gamma \delta^{a} \\
& \leq K \delta^{a+1} \leq K\left(R^{-1} \delta\right)^{a+1}|x|^{a+1} \lesssim|x|^{a+1} .
\end{aligned}
$$

As by hypothesis $|\mu(x)| \simeq|x|^{a}$, we may now return to (C-16) and write

$$
J_{0}(x)=P_{0}(\bar{x})+|\mu(x)| T_{0}(x),
$$

where $P_{0}$ is a polynomial of degree at most $a$, and the remainder $T_{0}$ is controlled by some constant depending on the $C^{1}$-norm of $u$ on $\partial B_{1}(0)$. Moreover, $T_{0}(x)=\mathrm{O}(|x|)$ near the origin.

We next estimate the integral $J_{1}$. To do so, we proceed as above and write

$$
J_{1}(x)=I_{1}(x)+\sum_{m=a+1}^{\infty} I_{2}^{m}(x)-\sum_{m=0}^{a} I_{1}^{m}(x)+\sum_{m=0}^{a} I_{1}^{m}(x)+I_{2}^{m}(x),
$$

where we have put

$$
\begin{aligned}
I_{1}(x) & :=\frac{1}{2 \pi} \int_{B_{1}(0) \cap B_{2|x|}(0)} \frac{x-y}{|x-y|^{2}} \mu(y) f(y) d y, \\
I_{1}^{m}(x) & :=\frac{1}{2 \pi} \int_{B_{1}(0) \cap B_{2|x|}(0)} P^{m}(x, y) \mu(y) f(y) d y, \\
I_{2}^{m}(x) & :=\frac{1}{2 \pi} \int_{B_{1}(0) \backslash B_{2|x|}(0)} P^{m}(x, y) \mu(y) f(y) d y .
\end{aligned}
$$

We first observe that the last sum in (C-18) may be written

$$
P_{1}(x):=\sum_{0 \leq m \leq a} I_{1}^{m}(x)+I_{2}^{m}(x)=\sum_{0 \leq m \leq a} \int_{B_{1}(0)} P^{m}(x, y) \mu(y) f(y) d y=\sum_{0 \leq m \leq a} A_{m} \bar{x}^{m},
$$


where

$$
A_{m}:=-\int_{B_{1}(0)} \bar{y}^{-(m+1)} \mu(y) f(y) d y .
$$

From the fact that $f \in L^{p}\left(B_{1}(0)\right)$ for $p>2$, and the hypothesis $|\mu(y)| \simeq|y|^{a}$, it follows easily that $\left|A_{m}\right|<\infty$ for $m \leq a$, and thus that $P_{1}$ is a polynomial of degree at most $a$.

We have next to handle the other summands appearing in (C-18), beginning with $I_{1}$. We find

$$
\begin{aligned}
\left|I_{1}(x)\right| & \lesssim|\mu(x)| \int_{B_{2|x|}(0)} \frac{|f(y)|}{|x-y|} d y \lesssim|\mu(x)| \int_{B_{3|x|}(x)} \frac{|f(y)|}{|x-y|} d y \\
& \lesssim|\mu(x)||x| M_{0} f(x) \lesssim|x|^{1-\frac{2}{p}}|\mu(x)|,
\end{aligned}
$$

where we have used the fact that $B_{2|x|}(0) \subset B_{3|x|}(x)$, and a classical estimate bounding convolution with the Riesz kernel by the maximal function ${ }^{23}$ (see [Ziemer 1989, Proposition 2.8.2]). We have also used the simple estimate $M_{0} f(x) \lesssim$ $|x|^{-2 / p}\|f\|_{L^{p}}$.

Next, let $q \in[1,2)$ be the conjugate exponent of $p$. We immediately deduce for $0 \leq m \leq a$ that

$$
\begin{aligned}
\left|I_{1}^{m}(x)\right| & \lesssim|x|^{m} \int_{B_{2|x|}(0)}|y|^{-1-m+a}|f(y)| d y \\
& \lesssim|x|^{a}\left\||y|^{-1}\right\|_{L^{q}\left(B_{2|x|}(0)\right)}\|f\|_{L^{p}\left(B_{1}(0)\right)} \lesssim|x|^{1-\frac{2}{p}}|\mu(x)| .
\end{aligned}
$$

We next estimate $I_{2}^{m}$. As $m \geq a+1$, we note that, for any $\epsilon>0$, we have

$$
a+1-m-\epsilon-\frac{2}{p}<0 .
$$

With again $q$ being the conjugate exponent of $p$, we find thus

$$
\begin{aligned}
\left|I_{2}^{m}(x)\right| & \lesssim|x|^{m} \int_{B_{1}(0) \backslash B_{2|x|}(0)}|y|^{a-1-m}|f(y)| d y \\
& =|x|^{m} \int_{B_{1}(0) \backslash B_{2|x|}(0)}|y|^{a+1-m-\epsilon-\frac{2}{p}}|y|^{\epsilon-\frac{2}{q}}|f(y)| d y \\
& \leq 2^{a+1-m-\epsilon-\frac{2}{p}}|x|^{a+1-\frac{2}{p}-\epsilon}\left\||y|^{\epsilon-\frac{2}{q}}\right\|_{L^{q}\left(B_{1}(0)\right)}\|f\|_{L^{p}\left(B_{1}(0)\right)} \\
& \lesssim 2^{a+1-m-\epsilon-\frac{2}{p}|x|^{1-\frac{2}{p}-\epsilon}|\mu(x)| .}
\end{aligned}
$$

Combining altogether in (C-18) our findings (C-19)-(C-21), we obtain that

$$
J_{1}(x)=P_{1}(\bar{x})+|\mu(x)| T_{1}(x),
$$

${ }^{23}$ See (C-8) for the definition of $M_{0} f$. 
where $P_{1}$ is a polynomial of degree at most $a$, and the remainder $T_{1}$ satisfies the estimate

$$
\left|T_{1}(x)\right| \lesssim|x|^{1-\frac{2}{p}-\epsilon} \text { for all } \epsilon>0 .
$$

Altogether, (C-17) and (C-22) put into (C-15) show that we have

$$
\nabla u(x)=P(\bar{x})+|\mu(x)| T(x),
$$

where $P:=P_{0}+P_{1}$ is a polynomial of degree at most $a$, and the remainder $T:=T_{0}+T_{1}$ satisfies the same estimate (C-23) as $T_{1}$. The announced statement (i) ensues immediately.

We prove next statement (ii). Comparing (C-14) to (C-24), we see that

(C-25) $|\mu(x)| Q(x)=\nabla(|\mu(x)| T(x))$

$$
=\nabla\left(|\mu(x)| T_{0}(x)\right)+\nabla I_{1}(x)+\sum_{m \geq a+1} \nabla I_{2}^{m}(x)-\sum_{0 \leq m \leq a} \nabla I_{1}^{m}(x) .
$$

By definition,

$$
|\mu(x)| T_{0}(x)=\sum_{m \geq a+1} C_{m} \bar{x}^{m},
$$

with the constants $C_{m}$ depending only on the $C^{1}$-norm of $u$ along $\partial B_{1}(0)$ and growing sublinearly in $m$. Using similar arguments to those leading to $(\mathrm{C}-17)$, it is clear from $(\mathrm{C}-11)$ that

$$
|\mu(x)|^{-1} \nabla\left(|\mu(x)| T_{0}(x)\right) \in L^{\infty}\left(B_{1}(0)\right) .
$$

Controlling the gradients of $I_{1}^{m}$ and $I_{2}^{m}$ is done mutatis mutandis the estimates (C-20) and (C-21). For the sake of brevity, we only present in detail the case of $I_{1}^{m}$. Namely,

$$
\begin{aligned}
& \text { (C-27) } \nabla I_{1}^{m}(x)=\frac{1}{2 \pi} \int_{B_{1}(0) \cap B_{2|x|}(0)} \nabla_{x} P^{m}(x, y) \mu(y) f(y) d y \\
& +\frac{1}{2 \pi} \frac{x}{|x|} \otimes \int_{\partial B_{2|x|}(0)} P^{m}(x, y) \mu(y) f(y) d y .
\end{aligned}
$$

After some elementary computations, and using the hypothesis $|\mu(y)| \simeq|y|^{a}$, we reach

$$
\begin{aligned}
\left|\nabla I_{1}^{m}(x)\right| & \lesssim m|x|^{a-2} \int_{B_{1}(0) \cap B_{2|x|}(0)}|f(y)| d y+|x|^{a-1} \int_{\partial B_{2|x|}(0)}|f(y)| d y \\
& \lesssim m|x|^{a-\frac{2}{p}}\|f\|_{L^{p}\left(B_{1}(0)\right)}+|x|^{a-1} \int_{\partial B_{2|x|}(0)}|f(y)| d y,
\end{aligned}
$$


so that immediately

$$
\left\||x|^{-a} \nabla I_{1}^{m}(x)\right\|_{L^{p-\epsilon}\left(B_{1}(0)\right)}<\infty \quad \text { for all } \epsilon>0 .
$$

Proceeding analogously for $\nabla I_{2}^{m}$, we reach that for any $\epsilon>0$ we have

$$
\sum_{m \geq a+1}\left\||x|^{-a} \nabla I_{2}^{m}(x)\right\|_{L^{p-\epsilon}\left(B_{1}(0)\right)}+\sum_{0 \leq m \leq a}\left\||x|^{-a} \nabla I_{1}^{m}(x)\right\|_{L^{p-\epsilon}\left(B_{1}(0)\right)}<\infty .
$$

Hence, there remains only to estimate $\nabla I_{1}$. This is slightly more delicate. For notational convenience, we write

$$
\nabla I_{1}(x)=\frac{1}{2 \pi} \nabla \int_{B_{1}(0) \cap B_{2|x|}(0)} \frac{x-y}{|x-y|^{2}} \mu(y) f(y) d y=: \frac{1}{2 \pi}(L(x)+K(x)),
$$

with

$$
K(x)=\chi_{B_{1 / 2}(0)}(x) \frac{x}{|x|} \otimes \int_{\partial B_{2|x|}(0)} \frac{x-y}{|x-y|^{2}} \mu(y) f(y) d y,
$$

and the convolution

$$
L(x)=\left(\Omega * f(y) \mu(y) \chi_{B_{1}(0) \cap B_{2|x|}(0)}(y)\right)(x),
$$

where $\Omega$ is the $(2 \times 2)$-matrix made of the Calderón-Zygmund kernels:

$$
\Omega(z):=\frac{|z|^{2} \rrbracket_{2}-2 z \otimes z}{|z|^{4}} .
$$

The boundary integral $K$ is easily estimated:

$$
|x|^{-a}|K(x)| \lesssim \frac{1}{|x|} \int_{\partial B_{2|x|}(0)}|f(y)| d y,
$$

thereby yielding

$$
\left\||x|^{-a} K(x)\right\|_{L^{p}\left(B_{1}(0)\right)} \lesssim\|f\|_{L^{p}\left(B_{1}(0)\right)} .
$$

To estimate $L$, we proceed as follows:

$(\mathrm{C}-31) \quad L(x)-\mu(x)\left(\Omega * f \chi_{B_{1}(0) \cap B_{2|x|}(0)}\right)(x)$

$$
=\int_{B_{1}(0) \cap B_{2|x|}(0)} \Omega(x-y) f(y)(\mu(y)-\mu(x)) d y .
$$

Let $S_{x}$ be the cone with apex the point $x / 2$ and such that the disk $B_{|x| / 4}(0)$ is inscribed in it. Note that, for $y \in S_{x}$, we have $2|x-y|>|x|$. Hence, we find

$$
\begin{aligned}
\int_{S_{x} \cap B_{1}(0) \cap B_{2|x|}(0)} \Omega(x-y) f(y)(\mu(y)-\mu(x)) d y & \\
& \lesssim|\mu(x)||x|^{-2} \int_{B_{2|x|}(0)}|f(y)| d y .
\end{aligned}
$$


By hypothesis, the function $\mu$ is continuously differentiable away from the origin. Thus, to each point $y$ in the complement of the cone $S_{x}$, there corresponds some $\alpha \equiv \alpha(x, y) \in[0,1]$ with

$$
\mu(y)-\mu(x)=(x-y) \cdot \nabla \mu(\alpha x+(1-\alpha) y) .
$$

Using (C-13), we deduce easily

$$
|\mu(y)-\mu(x)| \lesssim|x|^{a-1}|x-y| \quad \text { for all } y \in S_{x}^{c} \cap B_{1}(0) \cap B_{2|x|}(0) .
$$

Accordingly, we have

$$
\begin{aligned}
\int_{S_{x}^{c} \cap B_{1}(0) \cap B_{2|x|}(0)} \Omega(x-y) f(y)(\mu(y)-\mu(x)) d y & \\
& \lesssim|x|^{a-1} \int_{B_{2|x|}(0)} \frac{|f(y)|}{|x-y|} d y \lesssim|\mu(x)| M_{0} f(x),
\end{aligned}
$$

where we have used the same estimate as in (C-19). Bringing (C-32) and (C-33) into (C-31) and using the fact that $|\mu(x)| \simeq|x|^{a}$ yields

$$
\begin{aligned}
|\mu(x)|^{-1}|L(x)| \lesssim\left(\Omega * f(y) \chi_{B_{1}(0) \cap B_{2|x|}(0)}(y)\right)(x) & \\
& +\frac{1}{|x|^{2}} \int_{B_{2|x|}(0)}|f(y)| d y+M_{0} f(x) .
\end{aligned}
$$

Because $f$ is $L^{p}$, standard estimates on Calderón-Zygmund operators and on the maximal function, together with a classical Hardy inequality then give us

$$
\left\||\mu|^{-1} L\right\|_{L^{p}\left(B_{1}(0)\right)} \lesssim\|f\|_{L^{p}\left(B_{1}(0)\right)}<\infty .
$$

Owing to the latter and to (C-30), we obtain from (C-29) that $|\mu|^{-1} \nabla I_{1} \in L^{p}\left(B_{1}(0)\right)$. With (C-26) and (C-28), the identity (C-25) thus implies that $Q$ belongs to $L^{p-\epsilon}$ for all $\epsilon>0$. This completes the first part of statement (ii).

We shall now prove the second part of (ii), and show that the trace of $Q$ is in $L^{p}$. To this end, let us note that

$$
\operatorname{Tr} \nabla \bar{x}=\operatorname{Tr}\left(\begin{array}{rr}
1 & 0 \\
0 & -1
\end{array}\right)=0 .
$$

We have seen in (C-25) that

$$
|\mu| Q=\nabla\left(|\mu| T_{0}\right)+\nabla I_{1}+\sum_{m \geq a+1} \nabla I_{2}^{m}-\sum_{0 \leq m \leq a} \nabla I_{1}^{m} .
$$

By definition, $|\mu(x)| T_{0}(x)=\sum_{m \geq a+1} C_{m} \bar{x}^{m}$, so that (C-34) gives

$$
\operatorname{Tr} \nabla\left(|\mu(x)| T_{0}(x)\right)=0 .
$$


Owing to the fact that $P^{m}(x, y)=\bar{x}^{m} \bar{y}^{-(m+1)}$, it then easily follows from (C-34) and (C-27) that

$$
\operatorname{Tr} \nabla I_{1}^{m}(x)=\frac{1}{2 \pi} \operatorname{Tr} \frac{x}{|x|} \otimes \int_{\partial B_{2|x|}(0)} P^{m}(x, y) \mu(y) f(y) d y ;
$$

whence the estimate

$$
|\mu(x)|^{-1}\left|\operatorname{Tr} \nabla I_{1}^{m}(x)\right| \lesssim 2^{a-m-1} \frac{1}{|x|} \int_{\partial B_{2|x|}(0)}|f(y)| d y,
$$

and thus

$$
\left\||\mu|^{-1} \operatorname{Tr} \nabla I_{1}^{m}\right\|_{L^{p}} \lesssim 2^{a-m-1}\|f\|_{L^{p}} .
$$

In exactly the same fashion, one finds

$$
\left\||\mu|^{-1} \operatorname{Tr} \nabla I_{2}^{m}\right\|_{L^{p}} \lesssim 2^{a-m-1}\|f\|_{L^{p}} .
$$

Finally, there remains to handle the term $|\mu|^{-1} \operatorname{Tr} \nabla I_{1}$. But this term belongs to $L^{p}$, as we have shown that $|\mu|^{-1} \nabla I_{1}$ does. Using this in (C-35), together with (C-36)-(C-38), yields the announced result.

Corollary C.3. Let $u \in C^{2}\left(B_{1}(0) \backslash\{0\}\right)$ solve

$$
\Delta u(x)=\mu(x) f(x) \quad \text { in } B_{1}(0),
$$

where

$$
|f(x)| \lesssim|x|^{n+r} \text { and }|\mu(x)| \simeq|x|^{a}
$$

for two nonnegative integers $n$ and $a$, and $r \in(0,1)$.

Then

$$
\nabla u(x)=P(\bar{x})+|\mu(x)| T(x),
$$

where $P$ is a complex-valued polynomial of degree at most $(a+n+1)$, and near the origin $T(x)=\mathrm{O}\left(|x|^{n+1+r-\epsilon}\right)$ for every $\epsilon>0$.

If in addition $\mu$ satisfies (C-13), then $|x|^{-(n+r)}|\mu|^{-1} \nabla(|\mu| T)$ belongs to $L^{p}$ for all finite $p$. Furthermore, we have the estimate

$$
|\operatorname{Tr} \nabla(|\mu(x)| T(x))| \lesssim|x|^{n+r}|\mu(x)| .
$$

Proof. The argument goes along the same lines as that of Proposition C.2. We set

$$
\omega(x):=|x|^{n+r} \mu(x) \text { and } h(x):=|x|^{-(n+r)} f(x) .
$$

From the given hypotheses, we see that $h \in L^{\infty}$, and $\omega$ satisfies (C-11) with $(a+n+r)$ in place of $a$. If $\mu$ satisfies (C-13), then so does $\omega$, again with $(a+n+r)$ in place of $a$. 
Using the representation $(\mathrm{C}-15)$ gives

$$
\begin{aligned}
\nabla u(x)= & \frac{1}{2 \pi} \int_{\partial B_{1}(0)}\left[\frac{x-y}{|x-y|^{2}} \partial_{\vec{v}} u(y)-u(y) \partial_{\vec{v}} \frac{x-y}{|x-y|^{2}}\right] d \sigma(y) \\
& \quad-\frac{1}{2 \pi} \int_{B_{1}(0)} \frac{x-y}{|x-y|^{2}} \omega(y) h(y) d y \\
=: J_{0}(x)+J_{1}(x) & \text { for all } x \in B_{1}(0),
\end{aligned}
$$

where $\vec{v}$ is the outer normal unit vector to the boundary of $B_{1}(0)$.

The integral $J_{0}$ is estimated as in (C-17) so as to yield

$$
J_{0}(x)=P_{0}(\bar{x})+|\mu(x)| T_{0}(x),
$$

where $P_{0}$ is a polynomial of degree at most $(a+n+1)$, and $T_{0}(x)=\mathrm{O}\left(|x|^{n+2}\right)$ with $|\mu|^{-1} \nabla\left(|\mu| T_{0}\right)=\mathrm{O}\left(|x|^{n+1}\right)$.

We next estimate the integral $J_{1}$. We proceed again as we did in the proof of Proposition C.2. Namely,

$$
J_{1}(x)=I_{1}(x)+\sum_{m=a+n+2}^{\infty} I_{2}^{m}(x)-\sum_{m=0}^{a+n+1} I_{1}^{m}(x)+\sum_{m=0}^{a+n+1} I_{1}^{m}(x)+I_{2}^{m}(x),
$$

where we have put

$$
\begin{aligned}
I_{1}(x) & :=\frac{1}{2 \pi} \int_{B_{1}(0) \cap B_{2|x|}(0)} \frac{x-y}{|x-y|^{2}} \omega(y) h(y) d y, \\
I_{1}^{m}(x) & :=\frac{1}{2 \pi} \int_{B_{1}(0) \cap B_{2|x|}(0)} P^{m}(x, y) \omega(y) h(y) d y, \\
I_{2}^{m}(x) & :=\frac{1}{2 \pi} \int_{B_{1}(0) \backslash B_{2|x|}(0)} P^{m}(x, y) \omega(y) h(y) d y .
\end{aligned}
$$

As before, $P^{m}(x, y):=\bar{x}^{m} \bar{y}^{-(m+1)}$. We first observe that the last sum in the expression for $J_{1}$ may be written

$$
\begin{aligned}
P_{1}(x) & :=\sum_{0 \leq m \leq a+n+1} I_{1}^{m}(x)+I_{2}^{m}(x) \\
& =\sum_{0 \leq m \leq a+n+1} \int_{B_{1}(0)} P^{m}(x, y) \omega(y) h(y) d y=\sum_{0 \leq m \leq a+n+1} A_{m} \bar{x}^{m},
\end{aligned}
$$

where

$$
A_{m}:=\int_{B_{1}(0)} \bar{y}^{-(m+1)} \omega(y) h(y) d y .
$$

From the boundedness of $h$ and the hypothesis $|\omega(y)| \simeq|y|^{a+n+r}$, it follows easily that $\left|A_{m}\right|<\infty$ for $m<a+n+1+r$, and thus, since $r>0$, that $P_{1}$ is a polynomial 
of degree at most $(a+n+1)$. Once this has been observed, the remainder of the proof follows mutatis mutandis that of Proposition C.2. Namely, we write

$$
J_{1}(x)=P_{1}(\bar{x})+|\omega(x)| T_{1}(x),
$$

with $T_{1}(x)=\mathrm{O}\left(|x|^{1-\epsilon}\right)$ for all $\epsilon>0$. Moreover, $|\omega|^{-1} \nabla\left(|\omega| T_{1}\right) \in L^{p}$ for all $p<\infty$, and $|\omega|^{-1} \operatorname{Tr} \nabla\left(|\omega| T_{1}\right) \in L^{\infty}$.

Finally, setting $P=P_{0}+P_{1}$ and $T=T_{0}+|x|^{n+r} T_{1}=\mathrm{O}\left(|x|^{n+r+1-\epsilon}\right)$ gives the desired representation (C-39). Clearly, from (C-13) and the above, we have

$$
\left.\left.\left.|| \mu\right|^{-1} \nabla(|\mu| T)|\lesssim||\mu|^{-1} \nabla\left(|\mu| T_{0}\right)|+| x\right|^{n+r}|| \omega\right|^{-1} \nabla\left(|\omega| T_{1}\right) \mid,
$$

so that indeed $|x|^{-(n+r)}|\mu|^{-1} \nabla(|\mu| T)$ belongs to $L^{p}$ for all finite $p$. Furthermore, we have, as announced,

$$
\begin{aligned}
|\operatorname{Tr} \nabla(|\mu| T)| & \leq\left|\operatorname{Tr} \nabla\left(|\mu| T_{0}\right)\right|+\left|\operatorname{Tr} \nabla\left(|\omega| T_{1}\right)\right| \\
& \lesssim|x|^{n+1}|\mu|+|\omega| \lesssim|x|^{n+r}|\mu| .
\end{aligned}
$$

\section{References}

[Adams 1975] D. R. Adams, "A note on Riesz potentials", Duke Math. J. 42:4 (1975), 765-778. MR 56 \#16361 Zbl 0336.46038

[Almeida 1995] L. Almeida, "The regularity problem for generalized harmonic maps into homogeneous spaces", Calc. Var. Partial Differential Equations 3:2 (1995), 193-242. MR 97a:58040 Zbl 0820.58013

[Bernard and Rivière 2011a] Y. Bernard and T. Rivière, "Energy quantization for Willmore surfaces and applications", preprint, 2011. arXiv 1106.3780v1

[Bernard and Rivière 2011b] Y. Bernard and T. Rivière, "Local Palais-Smale sequences for the Willmore functional”, Comm. Anal. Geom. 19:3 (2011), 563-599. MR 2012j:58013 Zbl 1245.53051

[Burstall et al. 2002] F. E. Burstall, D. Ferus, K. Leschke, F. Pedit, and U. Pinkall, Conformal geometry of surfaces in $S^{4}$ and quaternions, Lecture Notes in Mathematics 1772, Springer, Berlin, 2002. MR 2004a:53058 Zbl 1033.53001

[Dautray and Lions 1984] R. Dautray and J.-L. Lions, Analyse mathématique et calcul numérique pour les sciences et les techniques, Masson, Paris, 1984. Translated as Mathematical analysis and numerical methods for science and technology, 3: Spectral theory and applications, Springer, Berlin, 1990. MR 91h:00004a Zbl 0642.35001

[Ejiri 1988] N. Ejiri, "Willmore surfaces with a duality in $S^{N}(1)$ ", Proc. London Math. Soc. (3) 57:2 (1988), 383-416. MR 89h:53117 Zbl 0671.53043

[Grüter and Widman 1982] M. Grüter and K.-O. Widman, "The Green function for uniformly elliptic equations", Manuscripta Math. 37:3 (1982), 303-342. MR 83h:35033 Zbl 0485.35031

[Hélein 1996] F. Hélein, Applications harmoniques, lois de conservation et repères mobiles, Diderot, Paris, 1996. Translated as Harmonic maps, conservation laws and moving frames, 2 nd ed., Cambridge Tracts in Mathematics 150, Cambridge University Press, Cambridge, 2002. MR 2003g:58024 Zbl 0920.58022

[Kuwert and Schätzle 2001] E. Kuwert and R. Schätzle, "The Willmore flow with small initial energy”, J. Differential Geom. 57:3 (2001), 409-441. MR 2003a:53097 Zbl 1035.53092 
[Kuwert and Schätzle 2004] E. Kuwert and R. Schätzle, "Removability of point singularities of Willmore surfaces”, Ann. of Math. (2) 160:1 (2004), 315-357. MR 2006c:58015 Zbl 1078.53007

[Kuwert and Schätzle 2007] E. Kuwert and R. Schätzle, "Branch points of Willmore surfaces", Duke Math. J. 138:2 (2007), 179-201. MR 2008j:53108 Zbl 1130.53007

[Li and Yau 1982] P. Li and S. T. Yau, "A new conformal invariant and its applications to the Willmore conjecture and the first eigenvalue of compact surfaces", Invent. Math. 69:2 (1982), 269-291. MR 84f:53049 Zbl 0503.53042

[Montiel 2000] S. Montiel, "Willmore two-spheres in the four-sphere", Trans. Amer. Math. Soc. 352:10 (2000), 4469-4486. MR 2001b:53080 Zbl 0961.53035

[Müller and Šverák 1995] S. Müller and V. Šverák, "On surfaces of finite total curvature”, J. Differential Geom. 42:2 (1995), 229-258. MR 97b:53007 Zbl 0853.53003

[Rivière 2008] T. Rivière, "Analysis aspects of Willmore surfaces", Invent. Math. 174:1 (2008), 1-45. MR 2009k:53154 Zbl 1155.53031

[Rivière 2010] T. Rivière, "Conformally invariant two-dimensional variational problems", preprint, Cours joint de l'Institut Henri Poincaré et Paris XII-Créteil, November 2010.

[Rivière 2013] T. Rivière, "Variational principles for immersed surfaces with $L^{2}$-bounded second fundamental form”, J. Reine Angew. Math. (2013). arXiv 1007.2997v1

[Tartar 2007] L. Tartar, An introduction to Sobolev spaces and interpolation spaces, Lecture Notes of the Unione Matematica Italiana 3, Springer, Berlin, 2007. MR 2008g:46055 Zbl 1126.46001

[Toro 1995] T. Toro, "Geometric conditions and existence of bi-Lipschitz parameterizations", Duke Math. J. 77:1 (1995), 193-227. MR 96b:28006 Zbl 0847.42011

[Weiner 1978] J. L. Weiner, “On a problem of Chen, Willmore, et al.”, Indiana Univ. Math. J. 27:1 (1978), 19-35. MR 57 \#7466 Zbl 0343.53038

[Ziemer 1989] W. P. Ziemer, Weakly differentiable functions: Sobolev spaces and functions of bounded variation, Graduate Texts in Mathematics 120, Springer, New York, 1989. MR 91e:46046 Zbl 0692.46022

Received April 27, 2012. Revised January 10, 2013.

YANN BERNARD

MATHEMATISCHES INSTITUT

ALBERT-LUDWIGS-UNIVERSITÄT

79004 FREIBURG

GERMANY

yann.bernard@math.uni-freiburg.de

TRISTAN RIVIÈRE

DEPARTMENT OF MATHEMATICS

ETH ZENTRUM

8093 ZURICH

SWITZERLAND

riviere@math.ethz.ch 


\title{
PACIFIC JOURNAL OF MATHEMATICS
}

\author{
msp.org/pjm
}

Founded in 1951 by E. F. Beckenbach (1906-1982) and F. Wolf (1904-1989)

\section{EDITORS}

V. S. Varadarajan (Managing Editor)

Department of Mathematics

University of California

Los Angeles, CA 90095-1555

pacific@math.ucla.edu

Paul Balmer

Department of Mathematics

University of California

Los Angeles, CA 90095-1555

balmer@math.ucla.edu

Daryl Cooper

Department of Mathematics

University of California

Santa Barbara, CA 93106-3080 cooper@math.ucsb.edu

Jiang-Hua $\mathrm{Lu}$

Department of Mathematics

Pokfulam Rd., Hong Kong jhlu@maths.hku.hk
The University of Hong Kong

Don Blasius

Department of Mathematics University of California

Los Angeles, CA 90095-1555

blasius@math.ucla.edu

Robert Finn

Department of Mathematics Stanford University

Stanford, CA 94305-2125

finn@math.stanford.edu

Sorin Popa

Department of Mathematics

University of California

Los Angeles, CA 90095-1555 popa@math.ucla.edu

Paul Yang

Department of Mathematics Princeton University

Princeton NJ 08544-1000

yang@math.princeton.edu

\section{PRODUCTION}

Silvio Levy, Scientific Editor, production@msp.org

\section{SUPPORTING INSTITUTIONS}

ACADEMIA SINICA, TAIPEI

CALIFORNIA INST. OF TECHNOLOGY

INST. DE MATEMÁTICA PURA E APLICADA

KEIO UNIVERSITY

MATH. SCIENCES RESEARCH INSTITUTE

NEW MEXICO STATE UNIV.

OREGON STATE UNIV.

\author{
STANFORD UNIVERSITY \\ UNIV. OF BRITISH COLUMBIA \\ UNIV. OF CALIFORNIA, BERKELEY \\ UNIV. OF CALIFORNIA, DAVIS \\ UNIV. OF CALIFORNIA, LOS ANGELES \\ UNIV. OF CALIFORNIA, RIVERSIDE \\ UNIV. OF CALIFORNIA, SAN DIEGO \\ UNIV. OF CALIF., SANTA BARBARA
}

\author{
Vyjayanthi Chari \\ Department of Mathematics \\ University of California \\ Riverside, CA 92521-0135 \\ chari@math.ucr.edu \\ Kefeng Liu \\ Department of Mathematics \\ University of California \\ Los Angeles, CA 90095-1555 \\ liu@math.ucla.edu \\ Jie Qing \\ Department of Mathematics \\ University of California \\ Santa Cruz, CA 95064 \\ qing@cats.ucsc.edu
}

These supporting institutions contribute to the cost of publication of this Journal, but they are not owners or publishers and have no responsibility for its contents or policies.

See inside back cover or msp.org/pjm for submission instructions.

The subscription price for 2013 is US \$400/year for the electronic version, and \$485/year for print and electronic.

Subscriptions, requests for back issues and changes of subscribers address should be sent to Pacific Journal of Mathematics, P.O. Box 4163, Berkeley, CA 94704-0163, U.S.A. The Pacific Journal of Mathematics is indexed by Mathematical Reviews, Zentralblatt MATH, PASCAL CNRS Index, Referativnyi Zhurnal, Current Mathematical Publications and the Science Citation Index.

The Pacific Journal of Mathematics (ISSN 0030-8730) at the University of California, c/o Department of Mathematics, 798 Evans Hall \#3840, Berkeley, CA 94720-3840, is published twelve times a year. Periodical rate postage paid at Berkeley, CA 94704, and additional mailing offices. POSTMASTER: send address changes to Pacific Journal of Mathematics, P.O. Box 4163, Berkeley, CA 94704-0163.

PJM peer review and production are managed by EditFLOW ${ }^{\circledR}$ from Mathematical Sciences Publishers.

\section{PUBLISHED BY}

mathematical sciences publishers

nonprofit scientific publishing

http://msp.org/

(C) 2013 Mathematical Sciences Publishers 


\section{PACIFIC JOURNAL OF MATHEMATICS}

Volume $265 \quad$ No. $2 \quad$ October 2013

Singularity removability at branch points for Willmore surfaces

YANN BERNARD and TRISTAN RIVIÈRE

On Bach flat warped product Einstein manifolds

QIANG CHEN and CHENXU HE

On plane sextics with double singular points

Alex DeGTyareV

A computational approach to the Kostant-Sekiguchi correspondence

HEIKo DiETRICH and WILLEM A. DE GRAAF

Landau-Toeplitz theorems for slice regular functions over quaternions

GRAZIANO GENTILI and GiUlia SARFATTI

On surgery curves for genus-one slice knots

PATRICK M. GILMER and CHARLES LIVINGSTON

Characterizing abelian varieties by the reduction of the Mordell-Weil group

Chris Hall and Antonella Perucca

Variation of complex structures and the stability of Kähler-Ricci

Solitons

StUART J. HALl and ThOMAS MURPHY

On crossed homomorphisms of the volume preserving diffeomorphism groups

RYOJI KASAGAWA

Regularity at the boundary and tangential regularity of solutions of the Cauchy-Riemann system

TRAN VU KHANH and GIUSEPPE ZAMPIERI

On the Steinberg character of a semisimple $p$-adic group 